\title{
Teoria degli anelli di serie di potenze.
}

\author{
Memoria di Walter Thimm (a Bonn, Germania)
}

Sunto. - In questa memoria è dato un riassunto della teoria degli anelli di serie di potenee con applieazioni nella geometria algebrica e nella teoria delle funzioni ai più variabili complesse. Uno sguardo alla prefazione darà un'idea più particolareggiata del contenuto.

\section{PREFAZIONE}

Questa memoria espone la teoria degli anelli di serie di potenze in vista di applicazioni alla geometria algebrica ed alla teoria delle funzioni di più variabili complesse. Essa si suddivide in due parti, la prima dedicata alla teoria delle serie di potenze formali, la seconda concernente le particolarita che intervengono quando si aggiunga il presupposto della convergenza.

Nella teuria delle serie di potenze formali ( $\$ \$ 1-14$ ) viene inizialmente supposto che il dominio dei coefficienti sia un anello commutativo dotato di unita; ma dal $\$ 9$ in poi si ammette che quello sia un corpo infinito, di guisa che il teorema di preparazione di WEIERstass risulta applicabile senza limitazioni. Strumento essenziale per dimostrare $\mathbf{i}$ teoremi più profondi sulle varieta algebroidi è la funzione norma, costitaente l'analogo della forma associata (o di CAYLEY) della geometria algebrica (cfr. [6], p. 32). Le applicazioni delle serie di potenze formali riguardano principalmente la geometria algebrica, e più precisamente lo studio di proprietà locali delle varietà algebriche. A questo scopo, nel $\$ 14$ si introduce il concetto di falda, investigandolo poi coll'uso della funzione norma.

Nella seconda parte, il dominio dei coefficienti e il corpo dei numeri complessi, e si possono utilizzare tutti i teoremi sulle serie di potenze formali anche nell'ipotesi della convergenza, in quanto le operazioni più impor. tanti del calcolo delle serie di potenze non conducono fuori dell' anello delle serie di potenze convergenti. A quei teoremi viene aggiunta una teoria dei germi di insiemi analitici, riferentesi alle proprietà di una varieta analitica nelle vicinanze di un punto fisso. Tali proprieta sono essenzialmente di natura topologica; ed anche ora il più importante strumento di dimostrazione è la funzione norma. 
Debbo l'iniziativa della presente memoria al Prof. Bentamino SEgre durante il mio soggiorno all' Istituto Nazionale di Alta Matematica in Roma, nell'anno accademico 1954-1955. Per questo, e per il Sno continuo interesse alla riuscita del lavoro gli sono molto obbligato. Ringrazio inoltre cordial. mente i Professori M. Benedictr e M. Rosatr, che sj sono sobbarcati alla non lieve fatica di tradurre il manoscritto tedesco in lingua italiana.

\section{§ 1. - Definizioni e notazioni.}

\subsection{Notazioni di teoria degli insiemi.}

Se $\mathbb{M D}$ e $\mathbb{H}$ 2 sono due insiemi, si indicherà con $\mathbb{M D} \cup \mathbb{H}$ la loro unione e

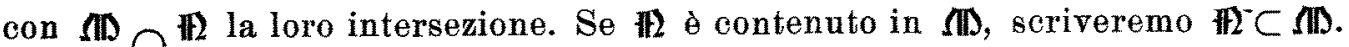
Se in particolare $P$ è un elemento dell' insieme $\Omega \mathbb{I}$, scriveremo $P \in \mathbb{I} \mathrm{D}$.

\subsection{Notazioni di teoria degli ideali.}

Tatti gli anelli che intervengono nella nostra trattazione sono commutativi.

(a) Gli ideali di un anello $\mathbb{R}$ saranno indicati con lettere gotiche minuscole $\mathfrak{a}, \mathfrak{b}, \ldots, \mathfrak{i}, \mathfrak{p}, \mathfrak{q}, \ldots$; inoltre le lettere $\mathfrak{p}$ e $\mathfrak{q}$ saranno riservate generalmente agli ideali primi o primari. L'ideale nullo sarà indicato con (0).

(b) Se $\mathfrak{a}$ e $\mathfrak{b}$ sono ideali dell anello $\mathbb{R}$, indicheremo con $(\mathfrak{a}, \mathfrak{b})$ l'ideale congiungente, cioè la totalità degli elementi $a+b$, con $a \in \mathfrak{a}$ e $b \in \mathfrak{b}$. Se $a_{1}, a_{2}, \ldots, a$, è una base dell' ideale $\mathfrak{a}$, scriveremo anche $\mathfrak{a}=\left(a_{1}, a_{2}, \ldots, a_{r}\right)$. $L$ 'ideale intersezione di $\mathfrak{a}$ e $\mathfrak{b}$ si indicherà con $\mathfrak{a} \cap \mathfrak{b}$; con $\mathfrak{a} \cdot \mathfrak{b}$ si indicherà l'ideale prodotto di $\mathfrak{a} e \mathfrak{b}$, cioè il più piccolo ideale che contiene tutti i prodotti $a b$, con $a \in \mathfrak{a}$ e $b \in \mathfrak{b}$.

(c) Un anello nel quale ogni ideale possiede una base finita si dice anello «noetheriano».

\subsection{Ideali ristretti $e$ ideali estesi.}

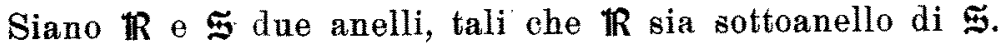

(a) Se $\mathfrak{a}$ e $\mathfrak{b}$ sono ideali di $\mathfrak{g}$, si ha (cfr. [7], pag. 36):

$$
(\mathfrak{a} \cap \mathbf{b}) \frown \mathbb{R}=(\mathfrak{a} \cap \mathbb{R}) \frown(\mathfrak{b} \cap \mathbb{R}) \text {. }
$$

(b) Se $\mathfrak{p}$ è un ideale primo o primario di $\mathfrak{s}$, allora anche $\mathfrak{p} \cap \mathbb{R}$ è ideale primo o rispettivamente primario di $\mathbb{R}$ (efr. [7], p. 37). 
(c) Sia a un ideale di tR. Le somme finite $\stackrel{r}{\Sigma}_{1}^{r} p_{i} a_{i}$, con $a_{i} \in \mathfrak{a}$ e $p_{i} \in \mathfrak{S}$ $(i=1,2, \ldots, r)$ riempiono an ideale di $\mathfrak{I}$, che si chiama l'estensione in $\mathfrak{I}$ dell' ideale $\mathfrak{a}$, o ideale esteso, e che si indica con $\mathfrak{g} \cdot \mathfrak{a}$. Valgono le relazioni (efr. [7], p. 34):

$$
\begin{aligned}
& \mathfrak{\xi} \cdot(\mathfrak{a} \cap \mathfrak{b}) \subset(\mathfrak{m} \cdot \mathfrak{a}) \cap(\mathfrak{m} \cdot \mathfrak{b}), \\
& \mathfrak{\Xi} \cdot(\mathfrak{a}, \mathfrak{b})=(\mathfrak{\Psi} \cdot \mathfrak{a}, \mathfrak{\Xi} \cdot \mathfrak{b}), \\
& \mathfrak{\Xi} \cdot(\mathfrak{a} \cdot \mathfrak{b})=(\mathfrak{m} \cdot \mathfrak{a}) \cdot(\mathfrak{m} \cdot \mathfrak{b}) .
\end{aligned}
$$

\subsection{Unità e ideali massimi.}

(a) Un elemento a dell'anello $\mathbb{R}$ si dice unità di $\mathbb{R}$ se l'equazione $a \cdot x=1$ ammette in $\mathbb{R}$ una soluzione $x$. Naturalmente $\mathbb{R}$ può contenere unità solo se ammette modulo, cioè un elemento identico (rispetto alla moltiplicazione). Un elemento di $\mathbb{R}$, che non sia un' unità, si dirà «non-unità. Le unita di un anello formano un gruppo moltiplicativo rispetto all'operazione di prodotto dell'anello. In un eorpo tutti gli elementi diversi dallo zero sono unità.

(b) Particolarmente importante è il caso nel quale le non-unità di $\mathbb{R}$ formano un'ideale in $\mathbb{R}$. Questa proprietà è collegata con una proprietà rela. tiva agli ideali massimi. Un ideale $m$ di un anello $\mathbb{R}$ si dice massimo in $\mathbb{R}$ se non esiste alcun ideale $a$ di $\mathbb{R}$, diverso da $\mathfrak{m}$ e da $\mathbb{R}$ stesso, $\theta$ tale che $\mathbf{m} \subset \mathbf{a} \subset \mathbb{R}$. Ebbene:

(c) Le non-unità di $\mathbb{R}$ formano in $\mathbb{R}$ un ideale se e soltanto se $\mathbb{R}$ possiede uno e un solo ideale massimo, e questo ideale consta allora dello non-unità di tR (cfr. [14], p. 48). locale».

(d) Un anello noetheriano con un solo ideale massimo si dice «anello

\subsection{Anelli di polinomi.}

Sia $\mathbb{R}$ un anello. L'anello dei polinomi in $n$ indeterminate $x_{1}, x_{2}, \ldots, x_{n}$ con coefficienti in $\mathbb{R}$ sarà indicato con $\mathbb{R}\left[x_{1}, x_{2}, \ldots, x_{n}\right]$. Brevemente scriveremo anche $\overline{\mathbb{R}}_{n}$, o, quando occorra tener presente la lettera usata per le variabili, $\overline{\mathbb{R}} . x$. Gli ideali di questo anello si indicheranno con $\overline{\mathfrak{a}}, \overline{\mathfrak{b}}, \ldots, \overline{\mathfrak{p}}, \overline{\mathfrak{q}}, \ldots$. 


\section{CAPITOLO $\mathrm{I}$}

\section{SERIE FORMALI DI POTENZE}

\section{§ 2. - Anelli di serie formali di potenze.}

2.1. Alla base delle considerazioni seguenti sta un anello commutativo $\mathbb{P}$. Sia $x_{1}, x_{2}, \ldots, x_{n}$ an sistema di indeterminate; indicheremo questo sistema con $x$, o, quando occorra tener conto del numero delle indeterminate, con $(x)_{n}$. Con $\varphi_{h}=\varphi_{h}(x)=p_{h}(x)_{n}=\varphi_{h}\left(x_{1}, x_{2}, \ldots, x_{n}\right)$ si denoterà un polinomio omogeneo di grado $h$ nelle indeterminate $x_{1}, x_{2}, \ldots, x_{n}$, con coefficienti in $\mathbb{D}$. Per serie formale di potenze intendiamo un'espressione del tipo:

$$
f(x)=f(x)_{n}=\varphi_{0}+\varphi_{1}(x)+\varphi_{2}(x)+\ldots+\varphi_{h}(x)+\cdots,
$$

nella quale $\varphi_{h}$ si chiama il termine di grado $h$ in $f$; in particolare $\varphi_{0}$ si dirà termine noto. Due serie formali di potenze si dicono identiche quando coincidono termine a termine.

2.2. Nell'insieme delle serie formali di potenze si definiscono una somma e un prodotto mediante le seguenti regole di calcolo: se $f=\varphi_{0}+\varphi_{1}+\ldots+\varphi_{h}+\ldots$ e $g=\psi_{0}+\psi_{1}+\ldots+\psi_{h}+\ldots$ sono le rappresentazioni di due serie formali di potenze, si ponga:

$$
f+g=\left(\varphi_{0}+\psi_{0}\right)+\left(\varphi_{1}+\psi_{1}\right)+\ldots+\left(\varphi_{n}+\psi_{h}\right)+\cdots
$$

e

2(3) $f \cdot g=\varphi_{0} \cdot \psi_{0}+\left(\varphi_{0} \psi_{1}+\varphi_{1} \psi_{0}\right)+\ldots+\left(\varphi_{0} \psi_{n}+\varphi_{1} \psi_{h-1}+\ldots+\varphi_{1} \psi_{n-i}+\ldots+\varphi_{h} \cdot \psi_{0}\right)+\ldots$.

Si verifica facilmente che, rispetto a queste operazioni, l'insieme delle serie formali di potenze è un anello commutativo. Esso si indicherà con $\mathbb{P}\left[\left[x_{1}, x_{2}, . ., x_{n}\right]\right]$, o brevemente con $\mathbb{P}_{n}$ o con $\mathbb{P}_{n} x$, efr. 1.5 .

2.3. L'anello delle serie di potenze $\mathbb{D}_{n}$ contiene tutti i polinomi nelle indeterminate $x$ con coefficienti in $\mathbb{P}$, perehè questi sono serie formali di potenze con un numero finito di termini diversi da 0 . Poichè le regole di calcolo $2(2)$ e $2(3)$ valgono anche per $i$ polinomi, l'anello $\mathbb{P}_{n}$ contiene come sottoanello l'anello dei polinomi $\overline{\mathbb{P}}_{n}$.

Nel seguito si studiano anelli di polinomi sopra anelli di serie di potenze e si adotterà la seguente notazione: l'anello dei polinomi nelle indeterminate $w_{1}, w_{2}, \ldots, w_{l}$ con coefficienti nell'anello delle serie di potenze $\mathbb{P}_{k}=\mathbb{P}\left[\left[z_{1}, z_{2}, \ldots, z_{k}\right]\right]$ si indicherà con $\mathbb{P}\left[\left[z_{1}, z_{2}, \ldots, z_{k}\right]\right]\left[w_{1}, w_{2}, \ldots, w_{l}\right] \quad 0$ brevemente con $\overline{\mathbb{D}}_{k, l}$. 
2.4. La struttura degli anelli di serie formali di potenze esce dall'ambito delle strutture usualmente considerate in algebra, perche per fissarne un elemento è necessaria una successione infinita di dati. Cio implica che in molte importanti questioni possono $\dot{\theta}$ debbono intervenire infinite operazioni di calcolo. Già la definizione di una serie formale di potenze fa apparire quest'ultima come il risultato di infinite addizioni. Poichè però nella presente situazione generale non si può parlare di convergenza, l'introduzione di infinite operazioni di calcolo con serie formali di potenze presuppone che, per ogni grado prefissato arbitrariamente, i relativi termini che compariscono nelle varie operazioni rimangano, da un certo punto in poi, inalterati. Per enunciare proposizioni in quest'ordine d'idee è necessaria la seguente defi. nizione.

Sia $f \in \mathbb{P}_{n}$ una serie formale di potenze $f \neq 0$. Sia $\varphi_{s}$ il primo termine non nullo nello sviluppo $2(1)$ di $f$; allora l'intero $s$ si dice il grado minimo di $f$ e si indica con $\omega(f) ; \varphi_{s}$ si ohiama il termine direttore di $f$. Se $f=0$ si pone $\omega(f)=\infty$.

Se $f, g$ sono elementi di $\mathbb{P}_{n}$, si ha:

$$
\omega(f+g) \geq \operatorname{minimo} \text { di } \omega(f) \text { e } \omega(g),
$$

e :

$$
\omega(f \cdot g) \geq \omega(f)+\omega(g)
$$

Se l'anello $\mathbb{P}$ d̀ un dominio d'integrità, ciò̀ se non possiede divisori dello zero, nella 2(5) vale sempre il segno di uguaglianza.

2.5. Teorema 1, cfr. [2], p. 58.

Sia $f_{1}, f_{2}, \ldots, f_{m}, \ldots$ una successione infinita di serie formali di potenze in $\mathbb{P}_{n}$. La successione dei loro gradi minimi $\omega_{1}, \omega_{2}, \ldots, \omega_{m}, \ldots$ tende verso $\infty$. Allora si può considerare la somma:

$$
f=f_{1}+f_{2}+\ldots+f_{m}+\cdots
$$

ed essa è una serie formale di potenze in $\mathbb{P}_{n}$.

Si fissi un intero $N \geq 0$. Dall'ipotesi sulla successione dei gradi minimi segue l'esistenza di un indice $h=h(N)$ tale che per $i \geq h$ si abbia $\omega_{i}>N$. Il termine di grado $N$ nella somma infinita $2(6)$ coincide allora col termine di grado $N$ della somma finita $f_{1}+f_{2}+\ldots+f_{h}$; ogni termine di $f$ si calcola dunque con un numero finito di addizioni. Ne segue il Teorema 1. 


\section{§ 3. - Sostituzioni nelle serie formali di potenze.}

3.1. Le serie formali di potenze non possono essere considerate come funzioni, ciò̀ non è lecito sostituire al posto delle $x$ un sistema di valori, se si eccettana ${ }^{\prime}$ valori (tutti $o$ in parte) nulli. Se infatti in una serie formale di potenze si pone lo zero al posto di talune delle $x$, rimane una serie formale di potenze nelle $x$ rimanenti. Però sono possibili anche altre sostituzioni, secondo la seguente definizione:

Siano $g_{1}, g_{2}, \ldots, g_{n}$ serie formali di potenze, prive di termine noto, nelle $m$ indeterminate $y_{1}, y_{2}, \ldots, y_{m}$ con coefficienti in $\mathbb{p}$ :

$$
g_{v} \in \mathbb{P}_{m} y, \quad \omega\left(g_{v}\right) \geq 1 \quad(v=1,2, \ldots, n)
$$

La sostituzione $(x)_{n}=T(y)_{m}$ di equazioni :

$$
x_{\nu}=g_{\nu}\left(y_{1}, y_{2}, \ldots, y_{m}\right) \quad(\nu=1,2, \ldots, n),
$$

si dice formalmente analitica in $\mathbb{P}_{m} y$. La matrice dei coefficienti dei termini lineari delle $g_{y}$ si denomina matrice funzionale di $T$. Nel caso in cui $m$ ed $n$ siano uguali, il determinante di questa matrice si chiama determinante funzionale di $T$ e lo si indica con $\Delta=\frac{\partial\left(x_{1}, x_{2}, \ldots, x_{n}\right)}{\partial\left(y_{1}, y_{2}, \ldots, y_{n}\right)}$; si ha $\Delta \in \mathbb{P}$.

\subsection{TeOREMa 2.}

Sia $f$ una serie formale di potenze di $\mathbb{P}_{n} x$. La trasformazione, formal. mente analitica in $\mathbb{D}_{m} y,(x)_{n}=T(y)_{m}$, trasforma $f$ in una serie formale di potenze $\bar{f}=\bar{f}\left(y_{1}, y_{2}, \ldots, y_{m}\right)=f\left(g_{1}, g_{2}, \ldots, g_{n}\right)$ dell'anello' $\mathbb{P}_{m} y$.

Se $f$ ̀̀ rappresentata mediante la 2(1), per la $2(4)$ e la 2(5) si ha che la serie formale di potenze $\bar{\varphi}_{h}=\bar{\varphi}_{h}(y)=\varphi_{h}\left(g_{1}, g_{2}, \ldots, g_{n}\right)$ ha grado minimo $\omega_{h} \geq h$. Applicando il Teorema 1 alla successione $\bar{\varphi}_{h}$ si ottiene il Teorema 2.

3.3. Una trasformazione analitica formale rappresenta omomorficamente l'anello delle serie di potenze $\mathbb{P}_{n} x$ nell'anello delle serie di potenze $\mathbb{P}_{m} y$. Si ha un isomorfismo tra questi due anelli quando $n=m$ e la trasformazione è invertibile. Per quanto riguarda la possibilità di tale inversione, si hanno i seguenti teoremi.

Se $(x)_{n}=T(y)_{m}$ designa una trasformazione analitica formale in $\mathbb{D}_{m} y$ e $(y)_{m}=U(z)_{p}$ una trasformazione analoga in $\mathbb{P}_{p} z$, in base al Teorema 2 si possono comporre le due trasformazioni e costruire la trasformazione $(x)_{n}=T U(z)_{p}$, che risulta analitica formale in $\mathbb{D}_{p} z$. La matrice funzionale 
di $T U$ è allora il prodotto delle matrici funzionali della $T$ e della $U$. In particolare, se il numero delle variabili $x, y, z$ ə lo stesso, si ha:

$$
\frac{\partial\left(x_{1}, x_{2}, \ldots, x_{n}\right)}{\partial\left(z_{1}, z_{2}, \ldots, z_{n}\right)}=\frac{\partial\left(x_{1}, x_{2}, \ldots, x_{n}\right)}{\partial\left(y_{1}, y_{2}, \ldots, y_{n}\right)} \cdot \frac{\partial\left(y_{1}, y_{2}, \ldots, y_{n}\right)}{\partial\left(z_{1}, z_{2}, \ldots, z_{n}\right)}
$$

3.4. Teorema 3: (Teorema sulle funzioni implicite), efr. [2], p. 64 .

Siano $F_{i}\left(x_{1}, x_{2}, \ldots, x_{n}, y_{1}, y_{2}, \ldots, y_{m}\right)(i=1,2, \ldots, m) m$ serie formali $d i$ potenze dell' anello $\mathbb{P}_{n+m}=\mathbb{P}\left[\left[x_{1}, x_{2}, \ldots, x_{n}, y_{1}, y_{2}, \ldots, y_{m}\right]\right]$. Queste $F_{1}$ siano prive di termine noto. Mediante la sostituzione $x_{1}=0, x_{2}=0, \ldots, x_{n}=0$ si ottiene da $F_{i}$ una serie formale di potenze nelle $y$; sia essa $f_{i}\left(y_{1}, y_{2}, \ldots, y_{m}\right) \in \mathbb{D}_{m} y$. Sia inoltre $\Delta$ il determinante funzionale $\Delta=\frac{\partial\left(f,, f_{2}, \ldots, f_{m}\right)}{\partial\left(y_{1}, y_{2}, \ldots, y_{m}\right)}$. Se $\Delta$ è un'unità di $\mathbb{P}$ (cfr. 1.4.) esistono $m$ serie formali di potenze dell' anello $\mathbb{P}_{n} x$ :

$$
y_{i}=g_{i}\left(x_{1}, x_{2}, \ldots, x_{n}\right) \quad(i=1,2, \ldots, m),
$$

tali che:

$$
F_{i}\left(x_{1}, x_{2}, \ldots, x_{n}, g_{1}, g_{2}, \ldots, g_{m}\right)=0 \quad(i=1,2, \ldots m) .
$$

Per la dimostrazione si introducano le seguenti notazioni. Sia $F \in \mathbb{P}_{n+m}$; sostituendo in $F \quad y_{1}=0, y_{2}=0, \ldots, y_{m}=0$ si ottenga $f \in \mathbb{P}_{n} x$. Il grado minimo di $f$ sia $\xi(F)$. Sia $\mathfrak{S}_{i, h}$ il termine incognito di grado $h$ nella rappresentazione di $g_{i}$; sia ciò̀ :

$$
g_{i}=\mathfrak{I}_{i, 1}+\mathfrak{S}_{i, 2}+\ldots+\mathfrak{I}_{i, h}+\ldots \quad(i=1,2, \ldots, m) .
$$

Determiniamo prima i $\mathfrak{\Xi}_{i, 1}$. Il termine lineare di $F_{i}$ sia :

$$
\sum_{j=1}^{m} a_{i j} y_{j}+\psi_{1,1} \quad(i=1,2, \ldots, m)
$$

ove $\psi_{i, 2}$ è una forma lineare nelle $x$ con coefficienti in $\mathbb{P}$. Le $\mathfrak{I}_{j, 1}(j=1,2, \ldots, m)$ sono allora determinate dal sistema:

$$
\sum_{j=1}^{m} a_{i j} \xi_{j, 1}+\psi_{i, 1}=0 \quad(i=1,2, \ldots, m) .
$$

Il determinante del sistema (2) è $\Delta$. Se $\Delta$ è un'unità di $\mathbb{P}$, si ha $\Delta^{-1} \in \mathbb{P}$ In questo caso la soluzione (2) ̀े costituita da forme lineari nelle $x$, con coeffi. cienti in $\mathbb{P}$ senza termine noto. Se per $j=1,2, . ., m$, si eseguisce nella $F_{i}$ la sostituzione

$$
\boldsymbol{y}_{i}=\mathfrak{s}_{j, 1}+y_{j}^{(1)}
$$


si ottiene una serie formale di potenze $F_{i}^{(1)}$ dell'anello $\mathbb{D}_{n+m}^{(1)}=\mathbb{D}\left[\left[x_{1}, x_{2}, \ldots, x_{n}\right.\right.$, $\left.\left.y_{1}^{(1)}, y_{2}^{(1)}, \ldots, y_{n}^{(1)}\right]\right]$ e si ha:

$$
F_{i}^{(1)}=\sum_{j=1}^{m} a_{i j} y_{j}^{(1)}+\Phi_{i}^{(1)} \quad(i=1,2, \ldots, m)
$$

Per le (1) e (2), $\Phi_{i}^{(1)}$ ha grado minimo $\geq 2$, e si ha anche $\xi\left(\Phi_{i}^{(1)}\right) \geq \dot{2}$.

Si supponga ora di aver calcolato $i$ termini di grado $\leq h$ nelle serie $g_{i}$. Questi termini soddisfacciano inoltre alle seguenti proprietà: $S e$, per $j=1,2, \ldots, m$ si esegue nelle $F_{i}$ la sostituzione:

$$
y_{j}=\mathfrak{I}_{j, 1}+\mathfrak{I}_{j, 2}+\ldots+\mathfrak{F}_{j, h}+\boldsymbol{y}_{j}^{(h)}
$$

si ottengono serie formali di potenze $F_{i}^{(h)}$ dell'anello $\mathbb{P}_{n+m}^{(h)}=\mathbb{D}\left[\left[x_{1}, x_{2}, \ldots, x_{n}\right.\right.$, $\left.\left.y_{1}^{(h)}, y_{2}^{(h)}, \ldots, y_{m}^{(h)}\right]\right]$ tali che:

$$
F_{i}^{(h)}=\sum_{j=1}^{m} a_{i j} y_{j}^{(h)}+\Phi_{i}^{(h)} \quad(i=1,2, \ldots, m)
$$

ove $\Phi_{i}^{(h)}$ ha grado minimo $\geq 2$ e $\xi\left(\Phi_{i}^{(h)}\right) \geq h+1$. Per $h=1$ si è già visto che queste proprietà valgono.

Se nella $\Phi_{i}^{(h)}$ sostituiamo le indeterminate $y_{j}^{(h)}$ con altrettanti zeri, otteniamo una serie formale di potenze di $\mathbb{P}_{n} x$, la quale, per la $\xi\left(\Phi_{i}^{(h)}\right) \geq h+1$, ha grado minimo $\geq h+1$; il suo termine di grado $h+1$ sia $\psi_{i, h+1}$. Allora le $\mathfrak{\Phi}_{j, h+1}$ sono date dal sistema :

$$
\sum_{j=1}^{m} a_{i j} \mathfrak{\Xi}_{j, h+1}+\psi_{i, h+1}=0 \quad(i=1,2, \ldots, m)
$$

Poichè $\Delta^{-1} \in \mathbb{D}$, si ottengono polinomi omogenei nelle $x$ di grado $h+1$ con coefficienti in $\mathbb{P}$. Se, per $j=1,2, \ldots, m$, eseguiamo nella $F_{i}^{(h)}$ la sostituzione:

$$
y_{j}^{(h)}=\mathfrak{\Xi}_{j, h+1}+y_{j}^{(h+1)},
$$

o il che e lo stesso, se effettuiamo la sostituzione:

$$
y_{j}=\mathfrak{\Xi}_{j, 1}+\mathfrak{\Xi}_{j, 2}+\ldots+\mathfrak{I}_{j, h+1}+y_{j}^{(h+1)} \quad(j=1,2, \ldots, \boldsymbol{m})
$$

sulla $F_{i}$, otteniamo una serie formale di potenze $F_{i}^{(h+1)}$ dell'anello $\mathbb{P}_{n+m}^{(h+1)}$, e si ha:

$$
F_{i}^{(h+1)}=\sum_{j=1}^{m} a_{i j} y_{j}^{(h+1)}+\Phi_{i}^{(h+1)} \quad(i=1,2, \ldots, m)
$$


Per le proprietà delle $\Phi_{i}^{(h)}$, la $\Phi_{i}^{(h+1)}$ ha grado minimo $\geq 2$, e in virtù delle $(4)$, ə $\xi\left(\Phi_{i}^{(h+1)}\right) \geq h+2$.

Abbiamo perciò la possibilità di calcolare con lo stesso metodo via via i termini di grado più alto e di ottenere così la serie $g_{i}$.

Come applicazioni di questo teorema si possono, come ora vedremo: (1) determinare le unità di $\mathbb{P}_{n}$, (2) caratterizzare le trasformazioni analitiche invertibili.

\subsection{TeOREMA 4.}

Una serie formale $f$ di potenze di $\mathbb{P}_{n}$ è un'unità quando e soltanto quando il suo termine noto è un' unità di $\mathbb{P}$.

(a) La necessità della condizione segue dalla definizione di unità.

(b) Per la dimostrazione dell' inverso poniamo $f=\Delta+f_{1}$, in modo che $f_{1} \in \mathbb{P}_{n}$ e $\omega\left(f_{1}\right) \geq 1$. Applichiamo poi il Teorema 3 alla serie $F=\Delta y+(y+1) f_{1}$. Poiche ora $\Delta$ è per ipotesi un' unità di $\mathbb{P}$, esiste una serie $g_{1} \in \mathbb{P}_{n} x$ tale che $\Delta g_{1}+\left(g_{1}+1\right) f_{1}=0$. Come si verifica facilmente, posto $g=\Delta^{-1}\left(g_{1}+1\right)$, vale la relazione $f \cdot g=1$, e quindi $f$ è un' unità.

Il Teorema 4 implica che gli elementi di $\mathbb{P}_{n}$ aventi grado minimo $\geq 1$ non sono certamente unità di $\mathbb{P}_{n}$. Se $\mathbb{P}$ è un corpo, tali elementi sono tutte e sole le non-unità. Più in generale, dal Teorema 4 e da 1.4. segue il

Teorema 5. - La totalità delle non-unità dell'anello di serie di potenze $\mathbb{P}_{n}$ è un ideale in $\mathbb{P}_{n}$ se e soltanto se la totalità delle non-unità dell'anello $\mathbb{P}$ forma un ideale in $\mathbb{D}$. In questo caso sia $\mathbb{P}$ che $\mathbb{P}_{n}$ sono anelli con uno ed un solo ideale massimo, l'ideale delle non-unità.

La condizione del teorema è soddisfatta p. es. quando $\mathbb{P}$ è un corpo, perchè allora lo zero è l'unica non-unità di $\mathbb{P}$, efr. 1.4. Se $\mathbb{P}$ è un corpo, l'ideale massimo delle non-unita di $\mathbb{P}_{n}$ ha la base $x_{1}, x_{2}, \ldots, x_{n}$.

\subsection{TeOREMa 6.}

Una trasformazione analitica formale in $\mathbb{P}_{n} y$ ì invertibile in $\mathbb{P}_{n} x$ se $e$ soltanto se il suo determinante funzionale è un' unità in $\mathbb{P}$.

(a) Per la dimostrazione della sufficienza di questa condizione basta applicare il Teorema 3 alle serie formali di potenze $F_{i}(x, y)=g_{i}(y)_{n}-x_{i}$ $(i=1,2, \ldots, n)$, ofr. $3(1)$, per $m=n$.

(b) Poichè la trasformazione identica ha determinante funzionale 1, la necessità segue dalla formula $3(2)$.

Una trasformazione analitica formale invertibile si dice «formalmente pseudoconforme». Una trasformazione formalmente pseudoconforme genera un isomorfismo tra gli anelli $\mathbb{P}_{n} x$ e $\mathbb{P}_{n} y$. Le trasformazioni formalmente 
pseudoconformi formano un gruppo, efr. 3.3. E particolarmente importante la ricerca di quelle proprietà degli anelli di serie formali di potenze e dei loro ideali che sono invarianti rispetto a tutte le trasformazioni formalmente pseudoconformi. Le chiameremo «invarianti analitici».

\section{§ 4. - Ideali in un anello di serie di potenze.}

4.1. Come in ogni anello, anche in un anello di serie di potenze si possono introdurre i concetti di ideale, ideale primo, ideale primario. Il più importante teorema in quest'ordine di idee è il seguente teorema della base.

Teorema 7 (di Rückert), cfr. [17].

Sia $\mathbb{P}$ un anello con modulo. L'anello di serie di potenze $\mathbb{D}_{n}$ è noẹtheriano, se $\mathbb{P}$ è un anello noetheriano.

$\dot{\mathrm{E}}$ sufficiente provare il teorema nel caso $n=1$. Se infatti esso vale in quest'ipotesi, la proprietà di essere noetheriano si trasporta da $\mathbb{D}$ a $\mathbb{P}_{1}$, quindi da $\mathbb{P}_{1}$ a $\mathbb{P}_{2}$, ecc. Sia a un ideale di $\mathbb{P}_{1}=\mathbb{P}[[x]]$. Gli elementi di a aventi grado minimo $k$ hanno termine direttore $b x^{k}$. L'insieme dei loro coefficienti, completato con lo zero, forma un ideale $\mathfrak{b}_{k}$ in $\mathbb{P}$. Poichè $1 \cdot x \in \mathbb{P}_{1}$ si ha che $\mathfrak{b}_{k} \subset \mathfrak{b}_{k+1}$. Essendo $\mathbb{P}$ noetheriano, la catena di ideali $\mathfrak{b}_{0} \subset \mathfrak{b}_{1} \subset \mathfrak{b}_{2} \subset \ldots$ ammette $\mathbf{u} \mathbf{n}$ elemento massimo $\mathfrak{b}_{m}=\mathfrak{b}_{m+1}=\ldots$. Per $k=0,1,2, \ldots, m$ sia $\mathfrak{b}_{3}^{(k)}, \mathfrak{b}_{2}^{(k)}, \ldots, \mathfrak{b}_{\alpha_{k}}^{(k)}$ una base di $\mathfrak{b}_{k}$. Esiste allora nna serie di potenze $f_{j}^{(k)} \in \mathbb{P}_{1}$ avente termine direttore $\mathfrak{b}_{j}^{(k)} x^{k}$. Queste serie di potenze $f_{j}^{(k)}\left(j=1,2, \ldots, \alpha_{A}\right.$; $k=0,1,2, \ldots, m$ ) formano una base di a; sia infatti $g \in \mathfrak{a}$, e il termine direttore di $g$ sia $b x^{s}$. Consideriamo prima il caso:

(1) $s \leq m$. Allora $b \in \mathfrak{b}_{s}$ e con opportuni elementi $h_{j}^{(s)} \in \mathbb{P}$ si ottiene $b=\sum_{j}^{\alpha_{s}} h_{j}^{(s)} \mathfrak{b}_{j}^{(s)}$. La serie di potenze $g^{(2)}=g-\sum_{j}^{\alpha_{s}} h_{j}^{(s)} f_{j}^{(s)}$ ha grado minimo $s_{2} \geq s+1$. Se è ancora $s_{2} \leq m$ si opera su $g^{j(2)}$ come si è operato su $g$, e cosi via. Dopo un numero finito di passi si ottiene una successione di elementi $g, g^{(2)}, \ldots, g^{(\omega)}$ di a con gradi minimi crescenti $s=s_{1}<s_{2}<\ldots<s_{\text {, }}$ e tale che $s_{r-1} \leq m<s_{r}$. Inoltre :

$$
g^{(r)}=g-\sum_{\lambda=1}^{r} \sum_{j=1}^{\alpha_{s_{\lambda}}} h_{j}^{\left(g_{\lambda}\right)} f_{j}^{\left(g_{\lambda}\right)}, \quad h_{j}^{\left(s_{\lambda}\right)} \in \mathbb{P}
$$

La serie $g^{(r)}$ presenta il caso

(2) $s>m$. Il termine direttore di $g^{(r)}$ sia $b^{(r)} x^{s}{ }^{s}$. Puichè $g^{(r)} \in \mathfrak{a}$ sỉ ha $b^{(r)} \in \mathfrak{b}_{s_{r}}=\mathfrak{b}_{m}$. Esistono quindi in $\mathbb{P}$ certi elementi $h_{j}^{\left(s_{r}\right)}$ tali che $b^{(r)}=\sum_{j=1}^{\alpha_{m_{m}}} h_{j}^{\left(s_{r}\right)} b_{j}^{(m)}$. 
Allora il grado minimo della serie $g^{(r+1)}=g^{(r)}-\left(\sum_{j=1}^{\alpha_{m}} h_{j}^{\left(s_{r}\right.} f_{j}^{(m)}\right) x^{s_{r}-m}$ è almeno $s_{r}+1$. Allo stesso modo operiamo su $g^{(r+1)}$ e così via; otteniamo una successione $g^{(r)}, g^{(r+1)}, \ldots$ con :

$$
g^{(v+1)}=g^{(\nu)}-\left(\sum_{j=1}^{\alpha_{m}} h_{j}^{\left(s_{y}\right)} f_{j}^{(m)}\right) x^{s_{\nu}-m}
$$

ove $h_{j}^{\left(s_{y}\right)} \in \mathbb{P}$ e $\mathrm{i}$ gradi minimi $s_{y} ; s_{\nu+1}$ di $g^{(\nu)}, g^{(y+1)}$ soddisfanno alla disuguaglianza $s_{v+1}>s_{y}$. Mediante le

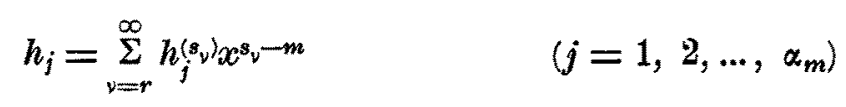

si definiscono certe serie formali di potenze di $\mathbb{P}_{1}$, per mezzo delle quali si può scrivere $g^{(r)}$ nella forma:

$$
g^{(r)}=\sum_{j=1}^{x_{m}} h_{j} f_{j}^{(m)}
$$

Da questa e dalla (1) si ottiene una rappresentazione di $g$ mediante gli ele. menti della base data.

4.2. Ricordando la definizione di anello locale (1.4., $d$ ), dai Teoremi 5 e 7 segue il

TeORema 8.

$L$ 'anello di serie di potenze $\mathbb{P}_{n}$ è un anello locale se tale è $\mathbb{P}$.

La condizione del teorema è p. es. soddisfatta se $\mathbb{D}$ è un corpo.

4.3. Come nella teoria generale degli ideali, si associa una dimensione ad ogni ideale primo di $\mathbb{D}_{n}: s i$ dice che un ideale primo $\mathfrak{p}$ di $\mathbb{P}_{n}\left(\mathfrak{p} \neq \mathbb{D}_{n}\right)$ ha dimensione $\rho$ se esiste una catena ascendente di ideali primi diversi:

$$
\mathfrak{p} \subset \mathfrak{p}_{1} \subset \ldots \subset \mathfrak{p}_{p}
$$

contenente $\rho+1$ termini e non ne esiste alcuna di lunghezza maggiore. Questa definizione si completa con la definizione di dimensione per ideali impuri : se $\mathfrak{a}$ è un ideale di $\mathbb{P}_{n}\left(\mathfrak{a} \neq \mathbb{P}_{n}\right)$ con la rappresentazione mediante ideali primari :

$$
\mathfrak{a}=\mathfrak{q}_{1} \frown \mathfrak{q}_{2} \frown \cdots \cap \mathfrak{q}_{r}
$$


e se $\mathfrak{p}_{i}$ è l'ideale primo associato all'ideale primario $\mathfrak{q}_{i}$, la dimensione di $\mathfrak{a}$ è per definizione la massima tra le dimensioni degli ideali primi $\mathfrak{p}_{i}$ $(i=1,2, \ldots, r)$.

Un'immediata conseguenza delle definizioni date è il seguente teorema, efr. 3.6.

Teorema 9.

La dimensione di un ideale in un anello di serie di potenze è un invariante analitico.

\section{§ 5. - Il lemma di Hensel.}

5.1. Per molte questioni sulla strnttura degli ideali di un anello di serie di potenze hanno grande interesse $i$ polinomi, con coefficienti in un tale anello. In quest' ordine di idee, fra i teoremi più notevoli, ricordiamo il lemma di Hensel e il teorema di preparazione di WeIERstrass.

Trorema 10 (Lemma di Hensel) (cfr. [20], pag. 52).

Sia $\mathbb{P}$ un corpo $e \mathbb{P}_{k}=\mathbb{P}\left[\left[x_{1}, x_{2}, \ldots, x_{k}\right]\right]$. Sia dato un polinomio $F\left(y \mid(x)_{k}\right)$ in $y$ dell'anello $\mathbb{P}_{k}[y]$ con coefficiente del termine di grado massimo uguale ad 1. Per $x_{1}=0, x_{2}=0, \ldots, x_{k}=0$ il polinomio $F$ sia il prodotto di due polinomi $g_{0}(y)$ e $h_{0}(y)$ di $\mathbb{P}[y]$ primi tra loro

$$
F\left(y \mid(0)_{k}\right)=g_{0}(y) \cdot h_{0}(y) .
$$

Essendo $m, p, q$ i gradi di $F, g_{0}, h_{0}$ risp., abbiamo $m=p+q$. Allora nell' $a$ nello $\mathbb{P}_{k}[y] F$ si scompone univocamente nel prodotto di due polinomi $G\left(y \mid(x)_{k}\right)$ di grado $p$, e $H\left(y \mid(x)_{k}\right)$ di grado $q$, tali che $G\left(y \mid(0)_{k}\right)=g_{0}(y)$ e $H\left(y \mid(0)_{k}\right)=h_{0}(y)$.

$$
F\left(y \mid(x)_{k}\right)=G\left(y \mid(x)_{k}\right) \cdot H\left(y \mid(x)_{k}\right),
$$

$G, H \in \mathbb{\mathfrak { p }}_{k}[y]$

Siano $X_{\lambda}^{(\nu)}, \lambda=1,2, \ldots, N_{\nu}^{\top}=\left(\begin{array}{c}\nu+k-1 \\ k-1\end{array}\right)$, i prodotti di potenze delle $x_{1}, x_{2}, \ldots, x_{k}$ di grado $\vee \geqq 1$. Ordiniamo $F\left(y \mid(x)_{k}\right)$ secondo questi prodotti di potenze:

$$
F\left(y \mid(x)_{k}\right)=g_{0}(y) \cdot h_{0}(y)+\sum_{\nu=1}^{\infty} \sum_{\lambda=1}^{N_{\nu}} X_{\lambda}^{(\nu)} f_{\lambda}^{(\nu)}(y),
$$


ove le $f_{\lambda}^{(\nu)}$ sono polinomi di $y$ in $\mathbb{P}[y]$ di grado $\leqq m-1$. Poniamo:

$$
\begin{aligned}
& G\left(y \mid(x)_{k}\right)=y_{0}(y)+\sum_{\nu=1}^{\infty} \sum_{\lambda=1}^{N_{\nu}} X_{\lambda}^{(\nu)} g_{\lambda}^{(\nu)}(y), \\
& H\left(y \mid(x)_{k}\right)=h_{0}(y)+\sum_{\nu=1}^{\infty} \sum_{\lambda=1}^{N_{\nu}} X_{\lambda}^{(\nu)} h_{\lambda}^{(\nu)}(y),
\end{aligned}
$$

e determiniamo le $g_{\lambda}^{(\nu)}, h_{\lambda}^{(\nu)}$ come polinomi in $y$ di gradi rispettivi $p-1$ e $q-1$, mediante un procedimento ricorrente. Perciò sia $g_{1}^{(0)}=g_{0}$, e $h_{1}^{(0)}=h_{0}$. Supponiamo che $g_{\lambda}^{(\nu)}$ e $h_{\lambda}^{(\nu)}$ siano già calcolati per $\nu=0,1, \ldots, r, \lambda=1,2, \ldots, s \leqq N_{\nu}$; per $\vee<r$ sia $s=N_{\nu}$. Distinguiamo i casi $s<N_{r}$, e $s=N_{,}$.

(1) Se $s<N_{r}$ determiniamo $g_{s+1}^{(r)}, h_{s+1}^{(r)}$ - secondo la seguente formula, che formalmente segue da $F=G \cdot H$ e dalle (2) e (3).

$$
g_{0} h_{s+1}^{(r)}+h_{0} g_{s+1}^{(r)}=f_{s+1}^{(r)}-\Sigma g_{\lambda}^{(\mu)} \cdot h_{\lambda^{\prime}}^{\left(\mu^{\prime}\right)}
$$

La somma a destra è estesa a tutti quei valori degli indici $\lambda, \mu, \lambda^{\prime}, \mu^{\prime}$, per i quali \& $X_{\lambda}^{(\mu)} \cdot X_{\left.\lambda \lambda^{\prime}\right)}^{\left(\mu^{\prime}\right)}=X_{s+1}^{(r)}$. Si ha inoltre $1 \leqq \mu \leqq r-1,1 \leqq \mu^{\prime} \leqq r-1$. Per la nostra premessa di induzione possiamo supporre noti tutti $i$ polinomi $g_{\lambda}^{(\mu)}, h_{\lambda^{\prime}}^{\left(\mu^{\prime}\right)}$ a secondo membro della (4).

(2) Nel caso $s=N_{r}, g_{1}^{(r+1)}$ e $h_{1}^{(r+1)}$ vengono espressi dalla relazione:

$$
g_{0} h_{1}^{(r+1)}+h_{0} g_{1}^{(r+1)}=f_{1}^{(r+1)}-\Sigma g_{\lambda}^{(\mu)} h_{\lambda^{\prime}}^{\left(\mu^{\prime}\right)}
$$

A secondo membro la somma è estesa a tutti i valori di $\lambda, \mu, \lambda^{\prime}, \mu^{\prime}$, per i quali ̀े $X_{2}^{(\mu)} \cdot X_{\lambda^{\prime}}^{\left(\mu^{\prime}\right)}=X_{1}^{(r+1)}$. Essendo $1 \leq \mu \leqq r, \quad 1 \leqq \mu^{\prime} \leqq r$, i polinomi corrispondenti sono noti.

Riassumendo: Nelle formule (4) e (5) i secondi membri sono polinomi noti di grado $\leq m-1$. La soluzione delle equazioni (4) $\Theta(5)$, per polinomi $g_{s+1}^{(r)}, h_{s+1}^{(r)}$ e $g_{1}^{(r+1)}, h_{1}^{(r+1)}$ di gradi rispettivi $p-1$ e $q-1$, equivale alla soluzione di un sistema di equazioni lineari, nel quale il determinante dei coefficienti è il risultante di $g_{0}$ e $h_{0}$. Ma questo risultante $\otimes$ diverso da 0 , perchè $g_{0}$ e $h_{0}$ non hanno un divisore comune. Cosicchè il calcolo dei polinomi $g_{s+1}^{(r)}, h_{s+1}^{(r)} \in g_{1}^{(r+1)}, h_{\mathrm{r}}^{(r+1)}$ è univocamente possibile. Abbiamo quindi un procedimento ricorrente per determinare tutti i polinomi $g_{\lambda}^{(\mu)}, h_{\lambda}^{(\mu)}$. Ordinando i secondi membri delle (2) $\Theta$ (3) per potenze di $y$, otteniamo i polinomi $G$ e $H$, che per $x_{1}=0, x_{2}=0, \ldots, x_{k}=0$ si riducono a $g_{0}$ e ad $h_{0}$. 
I $\S \S 3, " 4$ e 5 , contengono già risultati fondamentali per lo sviluppo della teoria. Ulteriori risultati dipendono dalla validita del teorema di preparazione di WeIERSTRass, del quale parleremo nel prossimo paragrafo.

\section{§ 6. - Il Teorema di preparazione di Welerstrass.}

6.1. Mediante il teorema di preparazione di Weierstrass si ottiene un' "algebrizzazione» dei problemi riguardanti le serie di potenze. $\grave{E}$ importante rilevare che la sua applicabilita resta subordinata ad un'ipotesi, nella quale una delle indeterminate comparisce in maniera diversa delle altre. Per mettere in evidenza questo fatto, denoteremo con $w$ tale indeterminata e con $z_{1}, z_{2}, \ldots, z_{k}$ le rimanenti. Il comportamento particolare dell'indeterminata $w$ è dato dalla seguente definizione:

(a) Sia $p\left(z_{1}, z_{2}, \ldots, z_{k}, w\right)$ una non-unità dell'anello $\mathbb{P}_{k+1}=\mathbb{P}\left[\left[z_{1}, z_{2}, \ldots\right.\right.$, $\left.\left.z_{k}, w\right]\right]$. Esiste un numero intero $s \geqq 1$ colla seguente proprietà. Se si ha:

$$
p=\sum_{\nu=0}^{\infty} p_{\nu}(z) w^{\nu}
$$

con $p_{y} \in \mathbb{P}_{k}=\mathbb{P}\left[\left[z_{1}, z_{2}, \ldots, z_{k}\right]\right]$, le serie $p_{0}, p_{1}, . .,, p_{s-1}$ abbiano grado minimo $\geq 1$; la serie $p_{s}$ abbia grado minimo 0 e il suo termine noto sia un' unità di ID. Diremo allora che $p$ è privilegiata rispetto a $w$.

(b) Un polinomio $\bar{p}$ dell'anello $\mathbb{D}_{k}[w]$ si dice privilegiato sopra $\mathbb{D}_{k}$ se si ha $($ con $m \geqq 1)$ :

$$
\bar{p}=w^{m}+a_{1}(z) w^{m-1}+\ldots+a_{m}(z)
$$

e i coefficienti $a_{i}(z)$ hanno grado minimo $\geq 1$. Per il polinomio privilegiato $\bar{p}$ usiamo il simbolo $\bar{p}(w \mid z)$.

Un polinomio in $w$, privilegiato sopra $\mathbb{p}_{k}$, $\doteq$ una (particolare) serie di potenze privilegiata rispetto a $w$ :

Possiamo anche considerare il caso in cui manchino le indeterminate $z_{i}$; denoteremo tale ipotesi con $k=0$.

In questo caso, una serie di potenze di $w$, di grado minimo $>1$ dovrà dirsi privilegiata rispetto a $w$ se e soltanto se il suo coeficiente del termine direttore è un' unita di $\mathbb{D}$; inoltre i polinomi privilegiati sopra $\mathbb{P}$ sono tutti e soli quelli della forma $w^{m}$, con $m \geq 1$.

6.2. Fondamentale per le successive ricerche è la così detta formula di Weingstrass, di cui ora dimostreremo la parte formale. 


\section{Teorema11 (Formula di Weiterstrass).}

Sia $p\left(z_{1}, z_{2}, \ldots . z_{k}, w\right)$ una serie di potenze dell'anello $\mathbb{P}_{k+1}=\mathbb{P}\left[\left[z_{1}, z_{2}, \ldots\right.\right.$, $\left.\left.z_{k}, w\right]\right]$; si supponga $p$ una non-unità privilegiata rispetto a $w$ e precisamente soddisfacente alle condizioni espresse in 6.1. (a). Consideriamo un'arbitraria serie di potenze di $\mathbb{p}_{k+1}$ :

$$
f=\sum_{\nu=0}^{\infty} f_{\nu}(z) w^{\nu}
$$

con $f_{\nu} \in \mathbb{P}_{k}=\mathbb{D}\left[\left[z_{1}, z_{2}, \ldots, z_{k}\right]\right]$. Esiste allora una serie di potenze $q$ in $\mathbb{P}_{k+1}$ e un polinomio $\bar{f}$ di $w$ con coefficienti in $\mathbb{P}_{k}$ e di grado $\leq s-1$ :

$$
\bar{f}=h_{0} w^{s-1}+h_{1} w^{s-2}+\ldots+h_{s-1}
$$

con $h_{\lambda}=h_{\lambda}(z) \in \mathbb{P}_{k}, \lambda=0,1,2, \ldots, s-1$, in modo tale che $i$ termini noti delle serie $h_{\lambda}$ e $f_{\lambda}(\lambda=0,1,2, \ldots, s-1)$ coincidono e si abbia

$$
p \cdot q+f=\bar{f} .
$$

Inoltre $q$ ed $\bar{f}$ sono univocamente determinati.

Poniamo:

$$
q=\sum_{\mu=0}^{\infty} q_{\mu}(z) w^{\mu}
$$

le nostre asserzioni seguono allora dall' univoca solubilità del sistema di equazioni :

$$
\sum_{\mu=0}^{m} q_{\mu} p_{m-\mu}+f_{m}=0 \quad \text { per } \quad m \geq s,
$$

che ora dimostreremo. Le serie di potenze considerate abbiano le seguenti rappresentazioni (cfr. 2.1):

$$
\begin{array}{ll}
p_{\nu}=\sum_{i=0}^{\infty} p_{v i}, & q_{\nu}=\sum_{i=0}^{\infty} q_{v i}, \\
f_{\nu}=\sum_{i=0}^{\infty} f_{v i}, & h_{\nu}=\sum_{i=0}^{\infty} h_{v i}
\end{array}
$$

In conseguenza delle nostre ipotesi valgono le : (2) $p_{00}=p_{10}=p_{20}=\ldots=p_{s-10}=0$ e $p_{s 0}=\mathfrak{g}$ è un'unita di $\mathbb{D}$. Allora le (1) forniscono: (3) $\sum_{\mu=0}^{m} \sum_{\nu=0}^{n} q_{\mu \nu} p_{m-\mu, n-\nu}+$ $+f_{m n}=0$ per $m \geq s, n \geq 0$. In particolare per $m=s, \stackrel{n=0}{n=0}=0$ si ottiene per le (2) la relazione:

$$
q_{00} \equiv+f_{80}=0
$$


Poichè per ipotesi $\mathfrak{I}$ è un'unità di $\mathbb{P}$, si ha :

$$
q_{00}=-f_{30} \Im^{-1}
$$

Attribuiamo ora al termine $q_{\mu \nu}$ l'indice intero $g(\mu, v)=\mu+v(s+1)$ e calco. liamo $i$ massimi indici dei termini di (3). Questi competono ai termini : $q_{m, n-1}, q_{m-s, n}, q_{m-s+1, n}, \ldots, q_{m n}$ e sono dunque $m+(n-1)(s+1)=$ $=m-s-1+n(s+1), m-s+n(s+1), \ldots, m+n(s+1)$. Per $j=1,2, \ldots, s$ nella (3) il termine $q_{n-s+j, n}$ è moltiplicato per $p_{s-j, 0}=0$. Supponiamo allora di conoscere tutte le $q_{\mu \nu}$ con $g(\mu, v)<m-s+n(s+1)$; la (3) permette di calcolare univocamente il termine $q_{m-s, n}$, che ha come coefficiente $p_{s 0}=\mathfrak{g}$. Poichè l'unico termine con indice 0 , ciò̀ $q_{00}$, è già stato ottenuto, abbiamo cosi un procedimento ricorrente per il calcolo dei termini $q_{\mu \nu}$ con indice $g(\mu, \nu)$ crescente, onde l'asserto.

\subsection{Una conseguenza della formula di WeIEnstrass è il}

Teorema 12 (Teorema di preparazione di Weierstrass).

Sia $p\left(z_{1}, z_{2}, \ldots, z_{k}, w\right)$ una serie di potenze dell'anello $\mathbb{D}_{k+1}=\mathbb{P}\left[\left[z_{1}, z_{2}, \ldots\right.\right.$, $\left.\left.z_{k}, w\right]\right]$. Inoltre $p$ sia una non-unità di $\mathbb{D}_{k+1}$, privilegiata rispetto $a w$ (cfr. 6.1.). Esistono allora un' unità $q$ dell'anello $\mathbb{D}_{k+1}$ e un polinomio privilegiato sopra $\mathbb{P}_{k} z$, sia esso $\bar{p}(w \mid z) \in \mathbb{P}_{k}[w]$, tali che si abbia

$$
p=\bar{p} \cdot q \text {. }
$$

Per la dimostrazione, applicando la formula di Wererstrass 6(4) con $f=-w^{x}$, si ha :

$$
q^{\prime} \cdot p-w^{*}=\bar{p}^{\prime}
$$

Per 6(5) il termine noto di $q^{\prime}$ è uguale a $\$^{-1}$. e, per il teorema 4 , $q^{\prime}$ e un'unità di $\mathbb{D}_{k+1}$. Se poniamo $\bar{p}=w^{s}+\bar{p}^{\prime}$, vale la $6(6)$ con $q=q^{\prime-1}$.

6.4. Una generalizzazione della formula di WeIERSTrass è data dal seguente :

\section{Teurema 13.}

Sia $\mathbb{P}_{k+i}$ l'anello di serie di potenze $\mathbb{P}\left[\left[z_{1}, z_{2}, \ldots, z_{k}, w_{1}, w_{2}, \ldots, w_{i}\right]\right]$ e $\left.\mathbb{P}_{k}=\mathbb{P} \mid\left[z_{1}, z_{2}, \ldots, z_{k}\right]\right]$. Per $i=1,2, \ldots, l$ sia dato un polinomio $p_{i}\left(w_{i} \mid z_{1}, z_{2}, \ldots, z_{k}\right.$, $\left.w_{1}, w_{s}, \ldots, w_{i-1}\right)$ di $w_{i}$, che sia privilegiato sopra $\mathbb{P}_{k+1-1}$. Allora ogni serie di potenze $f$ di $\mathbb{D}_{k+l}$ ammette una rappresentazione:

$$
f=q_{1} p_{1}+q_{2} p_{2}+\ldots+q_{l} p_{l}+\bar{f}
$$


nella quale $q_{j}$ appartiene all'anello $\mathbb{P}_{k+j}\left[w_{j+1}, w_{j+2}, \ldots, w_{l}\right](j=1,2, \ldots, l)$ e $\bar{f} \grave{e}$ un polinomio in $w_{1}, w_{2}, \ldots, w_{l}$ con coefficienti in $\mathbb{P}_{k}, \bar{f} \in \mathbb{P}_{k}\left[w_{1}, w_{2}, \ldots, w_{l}\right]$.

Per la formula di WeIersmass si ha $f=q_{l} p_{l}+f_{1}$, con $q_{l} \in \mathbb{D}_{k+l}$ e $f_{1} \in \mathbb{P}_{k+l-1}\left[w_{1}\right]$. Ammettiamo di aver già ottenuto per la $f$ una rappresentazione del tipo:

$$
f=q_{j+1} p_{j+1}+q_{j+2} p_{j+2}+\ldots+q_{l} p_{l}+f_{l-j},
$$

con $q_{\lambda} \in \mathbb{P}_{h+\lambda}\left[w_{\lambda+1}, w_{\lambda+2}, \ldots, w_{l}\right]$ e $f_{l-j} \in \mathbb{P}_{k+j}\left[w_{j+1}, w_{j+2}, \ldots, w_{l}\right]$. Come polinomio in $w_{j+1}, w_{j+2}, \ldots, w_{l}$, la $f_{l-j}$ ammette una rappresentazione del tipo:

$$
f_{l-1}=\sum_{y=1}^{n} a_{y} W_{y},
$$

ove $W_{\nu}$ ¿े un prodotto di potenze di $w_{j+1}, w_{j+2}, \ldots, w_{l}$ e $\mathrm{i}$ coefficienti $a_{v}$ appartengono a $\mathbb{P}_{k+j}$. Per la formula di Weirerstrass abbiamo:

$$
a_{v}=b_{\nu} p_{i}+\bar{a}_{v} \quad(v=1,2, \ldots, n)
$$

con $b_{y} \in \mathbb{P}_{k+j} \Leftrightarrow \tilde{a}_{y} \in \mathbb{P}_{k+j-1}\left[w_{j}\right]$. Ne segue :

$$
f_{l-j}=q_{j} p_{j}+f_{l-j+1},
$$

arendo posto $q_{j}=\sum_{\nu=1}^{n} b_{y} W_{\nu}$ e $f_{l-j+1}=\sum_{\nu=1}^{n} \tilde{a}_{\nu} W_{v}$. Si ha $q_{j} \in \mathbb{P}_{k+j}\left[w_{j+1}, w_{j+2}, \ldots, w_{l}\right]$ e $f_{l-j+1}$ è un polinomio dell'anello $\mathbb{P}_{k+i-1}\left[w_{j}, w_{j+1}, \ldots, w_{l}\right]$. Sostituendo (2) in (1) si ottiene $f=q_{j} p_{j}+q_{j+1} p_{j+1}+\ldots+q_{i} p_{l}+f_{l-j+1}$.

Abbiamo cosi un procedimento induttivo per il calcolo di $\bar{f}$, il quale procede per valori decrescenti di $j$ (cioè per $j=l-1, l-2, \ldots, 1,0$ ). Per $j=0$, dalla (l) si ottiene $\bar{f}=f_{l}$.

6.5. Molto importanti proprietà degli ideali di serie di potenze si ricavano mediante l'introduzione della cosi detta base polinomiale. All' nopo dimostriamo il seguente teorema:

\section{Teorema 14.}

Per $i \geq 1$ sia $\mathbb{P}_{k+i}$ l'anello di serie di potenze $\left.\mathbb{P}\left[z_{1}, z_{2}, \ldots, z_{k}, w_{1}, w_{2}, \ldots, w_{i}\right]\right]$, $e \mathbb{P}_{h}=\mathbb{P}_{k} z$. Sia $\mathfrak{a}$ un ideale in $\mathbb{P}_{k+l}, \mathfrak{a} \neq \mathbb{P}_{k+l}$, il quale ammetta base finita e goda della seguente proprietà: Per $i=1,2, \ldots, l, l$ 'ideale a contenga un polinomio $p_{1}\left(w_{i} \mid z_{1}, z_{2}, \ldots, z_{k}, w_{1}, w_{2}, \ldots, w_{i-1}\right)$ nella $w_{i}$, che sia privilegiato sopra $\mathbb{P}_{k+i-1}$. Allora a possiede una base nell'anello $\left.\mathbb{P}_{k} \mid w_{1}, w_{2}, \ldots, w_{1}\right]$, cioè nell'anello dei polinomi in $x_{1}, w_{2}, \ldots, w_{l}$ con coefficienti in $\mathbb{D}_{k} z$. 
Sia $f_{1}, f_{2}, \ldots, f_{r}$ una base di a. Per la formula di Wererstrass si ha:

$$
f_{\lambda}=q_{\lambda} p_{l}+\bar{f}_{\lambda} \quad(\lambda=1,2, \ldots, r)_{,}
$$

con $q_{\lambda} \in \mathbb{P}_{k+l}$ e $\bar{f}_{\lambda} \in \mathbb{P}_{k+l-1}\left[w_{l}\right]$. Di conseguenza $\bar{f}_{1}, \bar{f}_{2}^{-}, \ldots, f_{r}^{-}, p_{l}$ costituiscono una base di a in $\mathbb{\mathbb { P }}_{k+l-1}\left[w_{l}\right]$. Ammettiamo di aver già costruito una base $g_{1}, g_{2}, \ldots, g_{s}$ di a nell'anello $\mathbb{1}_{k+j}\left[w_{j+1}, w_{j+2}, \ldots, w_{l}\right]$. Come nella dimostrazione del Teorema 13 , si trovano certi elementi $h_{\lambda}$ di $\mathbb{P}_{k+j}\left[w_{j+1}, w_{j+2}, \ldots, w_{\ell}\right]$ e certi polinomi $\bar{g}_{\lambda} \in \mathbb{D}_{k+j-1}\left[w_{j}, w_{j+1}, \ldots, w_{l}\right]$ tali che:

$$
g_{\lambda}=h_{\lambda} p_{j}+\bar{g}_{\lambda} \quad(\lambda=1,2, \ldots, s)
$$

Gli elementi $\bar{g}_{1}, \bar{g}_{2}, \ldots, \bar{g}_{s}, p_{j}$ costituiscono una base di a nell' anello $\mathbb{D}_{k+j-1}\left[w_{j}, w_{j+1}, \ldots, w_{l}\right]$. Si costruisce cosi, in forma ricorrente rispetto all'indice $j$ decrescente, una base di a nell'anello $\mathbb{D}_{k}\left[w_{1}, w_{2}, \ldots, w_{l}\right]$.

Corollario. - Sotto le ipotesi del teorema 14, l'ideale a contiene per ogni $i=1,2, \ldots, l$ un polinomio $\bar{p}_{i}$ dell' anello $\mathbb{P}_{k}\left[w_{1}, w_{2}, \ldots, w_{t}\right]$, il quale come polinomio in $w_{i}$ è privilegiato sopra $\mathbb{P}_{k+i-1}$. (Si osservi che abbiamo soltanto supposto $p_{i} \in \mathbb{P}_{k+i-1}\left[w_{i}\right]$ ).

Sia

$$
p_{i}=w_{i}^{m}+\sum_{\lambda=0}^{m-1} a_{\lambda}\left((z)_{k}, w_{1}, \ldots, w_{i-1}\right) w_{i}^{\lambda}
$$

Se applichiamo il teorema 13 ai coefficienti $a_{\lambda}$ consegue:

$$
a_{\lambda}=\sum_{\mu=1}^{i-1} b_{\lambda \mu} p_{\mu}+\bar{a}_{\lambda}
$$

con $b_{\lambda \mu} \in \mathbb{D}_{k+\mu}\left[w_{\mu+1}, \ldots, w_{i-1}\right]$ e $\tilde{a}_{\lambda} \in \mathbb{P}_{k}\left[w_{1}, \ldots, w_{i-1}\right]$. Dalle (1) e (2) si trae

$$
p_{i}=\sum_{\mu=1}^{i-1} q_{\mu} p_{\mu}+\bar{p}_{i}
$$

$\operatorname{con} q_{\mu}=\sum_{\lambda=0}^{m-1} b_{\lambda \mu} v_{i}^{\lambda}$ e $\bar{p}_{i}=w_{i}^{m}+-\sum_{\lambda=0}^{m-1} \bar{a}_{\lambda} w_{i}^{\lambda} ;$ dunque $\bar{p}_{i} \in \mathbb{P}_{h}\left[w_{1}, w_{2}, \ldots, w_{i}\right]$. Se sostituiamo in (3) $z_{1}=0, z_{2}=0, \ldots, z_{k}=0, w_{1}=0, w_{2}=0, \ldots, w_{i-1}=0$, a destra $p_{1}, p_{2}, \ldots, p_{i_{-1}}$ si annullarano, e quindi anche la somma. Percio accanto a $p_{i}$, anche $\vec{p}_{i}$ eे privilegiato sopra $\mathbb{P}_{k+i-1}$. 


\section{§ 7. - Sulla decomposizione in fattori di serie di potenze e polinomi privilegiati.}

7.1. Il teorema di preparazione di WEIERSTRAss pone in evidenza la classe delle serie di potenze privilegiate e dei polinomi privilegiati; dedurremo qui talune loro proprietà.

TEOREMa 15.

Sia $\mathbb{P}$ un dominio d'integrità. (a) Se $p$ e $q$ sono due serie di potenze dell'anello $\mathbb{P}_{k+1}=\mathbb{P}\left[\left[z_{1}, z_{2}, \ldots, z_{k}, w\right]\right]$, privilegiate rispetto $a w$, allora anche $i l$ loro prodotto $h=p \cdot q$ è privilegiato rispetto $a w$. (b) Se $h$ è una serie di potenze dell'anello $\mathbb{P}_{k+1}$, privilegiata rispetto a $w$, e se $h=p \cdot q$, ove $p$ e $q$ appartengono $a \mathbb{P}_{k+1}$, allora anche $p$ e $q$ sono privilegiati rispetto $a w$.

Se in $h, p, q$ eseguiamo la sostituzione $z_{1}=0, z_{2}=0, \ldots, z_{k}=0$, si ha

$$
h(0, w)=p(0, w) \cdot q(0, w)
$$

Poichè $\mathbb{P}$ è un dominio di integrità, nessuna di queste serie si annulla sotto le ipotesi (a) o (b). I loro termini direttori siano rispettivamente $\rho w^{r}, \sigma w^{s}, \tau w^{t}$ $(r \geqq 1, s \geqq 1, t \geqq 1)$; si ha allora $r=s+t$ e $\rho=\sigma \tau$. Per quanto riguarda la parte $(a)$ del teorema, $\sigma$ e $\tau$ sono unita di $\mathbb{P}$, quindi lo è anche $\rho$. Per la parte (b), $\rho$ è supposto un' unità di $\mathbb{D}$; ne segue $1=\sigma \tau \rho^{-1}$, e da cio risulta che anche $\rho$ e $\sigma$ sono unità.

7.2. Accanto al teorema precedente, sulle serie di potenze privilegiate, poniamo il seguente teorema sui polinomi privilegiati:

Teorema 16.

Sia $\mathbb{P}$ un dominio d'integrità. (a) Siano $\bar{p}$ e $\bar{q}$ due polinomi in $w$, privilegiati sopra $\mathbb{P}_{k} z$; allora lo è anche $\bar{h}=\bar{p} \cdot \bar{q}$. (b) Sia $\bar{h}$ un polinomio in $w$, privilegiato sopra $\mathbb{P}_{k} z$; se si ha $\bar{h}=\bar{p} \cdot \bar{q}$, essendo $\bar{p}$ e $\bar{q}$ polinomi dell'anello $\mathbb{P}_{k}[w]$, allora questi si possono ridurre, mediante moltiplicazione per unità di $\mathbb{P}_{\mathrm{h}} z$, a polinomi privilegiati sopra $\mathbb{P}_{h} z$.

(a) La parte (a) segue immediatamente dalle definizioni.

(b) Poichè il prodotto dei coefficienti dei termini di grado massimo di $\bar{p}$ e $\bar{q}$ dà il coefficiente del termine di grado massimo di $\bar{h}$, il quale è 1 , i primi sono unità di $\mathbb{D}_{k}$. Mediante moltiplicazione per unità di $\mathbb{D}_{k}$ possiamo ridurli all'identita. Se sostituiamo, in $\bar{h}, z_{1}=z_{2}=\ldots=z_{k}=0$, otteniamo una potenza di $w$; quindi anche $p$ e $\bar{q}$ devono ridursi con questa sostituzione ad una potenza di $w$. A prescindere dal coefficiente del termine di grado massimo, i coefficienti di $\frac{p}{p}$ di $\bar{q}$ hanno perciò tutti grado minimo $\geq 1$. 
7.3. Dopo questi preliminari possiamo dimostrare taluni teoremi sulla decomposizione in fattori. In molti anelli, che si presentano spesso, vale il teorema dell'univoca decomponibilità in fattori: Se $a$ e $b$ sono non-unità dell'anello $\mathbb{R} ; a$ si dice divisibile per $b$ se esiste un elemento $c$ di tR tale che $a=b \cdot c ; b$ si dice divisore proprio di $a$ se anche $c$ è una non-unità; l'elemento $a \mathrm{di}$. $\mathbb{R}$ si dice irriducibile se non ammette divisori propri. Il teorema dell'univoca decomponibilità in fattori si enuncia come segue:

Ogni elemento di $\mathbb{R}$ è rappresentabile come prodotto di un numero finito di elementi irriducibili di tR. Questa decomposizione è univoca a meno di unità di $\mathbb{R}$.

Il problema della decomposizione in fattori negli anelli di serie di potenze è molto più complicato che negli anelli di polinomi, e non è ancora risoluto in generale. Negli anelli di polinomi vale, com'e noto, il teorema (cfr. [4], p. 6) :

Se tR è un'anello nel quale vale il teorema dell'nnivoca decomponibilità in fattori, tale teorema vale anche in ogni anello di polinomi $\mathbb{R}[x]$.

Nella dimostrazione dell' univocità interviene in maniera essenziale l'al. goritmo euclideo delle divisioni successive. Poichè negli anelli di serie di potenze non vale alcunchè di analogo, il teorema dell'univoca decomponibili à può essere dimostrato soltanto sotto opportune ipotesi restrittive per $\mathbb{P}$.

\section{Teorema 17.}

Sia $\mathbb{P}$ un dominio d'integrilà. Nell'anello di serie di potenze $\mathbb{P}_{k} z$ valga il tcorema dell'univoca decomponibilità in fattori. Allora ogni serie dell'anello $\mathbb{P}_{k+1}=\mathbb{P}\left[\left[z_{1}, z_{2} \ldots, z_{k}, w\right]\right]$ la quale sia privilegiala rispelto a $w$, aminette una decomposizione in faltori univoca.

La serie $p \in \mathbb{P}_{h+1}$ sia privilegiata rispetto a $w$; per il teorema di preparazione di Weierstrass essa è il prodotto di un'unita di $\mathbb{P}_{k+1}$ per un polinomio $\bar{p}$, privilegiato sopra $\mathbb{P}_{k}$. Per il Teorema 15 lo stesso fenomeno si verifica per ogni fattore di $p$ appartenente a $\mathbb{D}_{k}$. $_{1}$. Ad una decomposizione in fattori di $\boldsymbol{p}$ in $\mathbb{P}_{k+1}$ corrisponde dunque un' "naloga decomposizione di $\bar{p}$ in $\mathbb{P}_{k}[w]$, e da un fattore dell'una decomposizione si passa al corrispondente nell'altra mediante moltiplicazione p $\cdot r$ unita $d i \mathbb{P}_{k+1}$. Inversamente per il Teorema 16, ogni decomposizione in fattori di $\bar{p}$ in $\mathbb{P}_{k}[w]$ dà luogo ad una decomposizione di $p$ in $\mathbb{P}_{A+1}$. Il problema della decomposizione in fattori di $p$ è cosi ricondotto alla determinazione dei fattori irriducibili di $\bar{p}$ in $\mathbb{P}_{k}[\boldsymbol{w}]$. Dall'ipotesi dell'univoca decomponibilita in fattori in $\mathbb{P}_{k}$ segue quaindi, per il teorema di algebra sopra citato, l'analoga proprietà in $\mathbb{P}_{k}[w]$. In particolare $\bar{p}$ ammette in quest'anello una decomposizione univoca in fattori irriducibili, e quindi l'ammette anche $p$ in $\mathbb{D}_{k+1}$. 
7.4. Il Teorema 17 mostra chiaramente quale importanza abbia il teorema di preparazione di WEIERSTRASS nella teoria della decomposizione in fattori. Naturalmente sarebbe sufficiente che la serie considerata fosse privilegiata rispetto a un'altra delle indeterminate. La questione rimane percio aperta solo per le serie di potenze che non sono privilegiate rispetto ad alcuna delle indeterminate. In molti casi importanti possono essere d'aiuto, in tale circostanza, le considerazioni seguenti. Poniamo la seguente definizione:

Una trasformazione lineare omogenea $(y)_{,}=L(x)_{n}$ :

$$
y_{i}=a_{i 1} x_{1}+a_{i 2} x_{2}+\ldots+a_{i n} x_{n} \quad(i=1,2, \ldots, n)
$$

con coefficienti $a_{i j}$ in $\mathbb{P}$ e determinante dei coefficienti non nullo si chiama "D-trasformazione di coordinate».

TeOREMa 18.

Sia IP un corpo con infiniti elementi. Siano $f_{1}, f_{2}, \ldots, f_{m} m$ non-unità dell'anello $\mathbb{P}_{n} x$. Allora esiste una $\mathbb{P}$-trasformazione di ccordinate $7(1)$ tale che le serie trasformate $\bar{f}_{i}(y) \in \mathbb{P}_{n} y(i=1,2, \ldots, m)$ siano privilegiate rispetto all' indeterminata $y_{n}$.

Introducendo nuove indeterminate $u_{1}, u_{2}, \ldots, u_{n}$, eseguiamo in $f_{\lambda}(x)$ $(\lambda=1,2, \ldots, m)$ la sostituzione

$$
\begin{aligned}
& x_{i}=y_{i}+u_{i} y_{n} \quad(i=1,2, \ldots, n-1), \\
& x_{n}=\quad u_{n} y_{n}
\end{aligned}
$$

Se $\varphi_{r_{\lambda}}^{(\lambda)}$ è il termine direttore di $f_{\lambda}, \bar{f}_{\lambda}$ possiede un termine direttore di grado

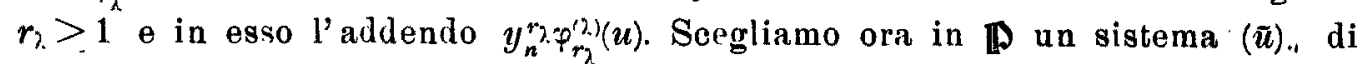
valori tale che si abbia $\bar{u}_{n} \varphi_{r_{1}}^{(1)}(\bar{u}) \cdot f_{r_{3}}^{(2)}(\tilde{u}) \cdot \ldots \cdot \varphi_{r_{m}}^{(m)}(\bar{u}) \neq 0$. Poichè $\mathbb{P}$ contiene infiniti e'ementi, un tale sistema esiste. Ponendo in (1) tali valuri ne segue l'asserto.

7.5. Sotto le ipntesi del Toorema 18 ogni non-unita di $\mathbb{P}_{n}$ può essere ricnndotta, con una $\mathbb{P}$-trasformazione di courdinate, ad una serie che sia privilegiata rispetto ad una delle indeterminate. Ora si può dunque dedurre senza limitazioni l'univoca decomponibilità in fattori in $\mathbb{P}_{n}$ dall'ipotesi che essa valga in $\mathbb{P}_{n-1}$. Ne segue:

Teorema 19.

Se $\mathbb{P}$ è un corpo con infiniti elementi, allora in ogni anello di serie di potenze $\mathbb{P}_{n}$ vale il teorema dell'univoca decomponibilità in fattori (cfr. 7.3.). 
Rimane aperta la questione della validità di tale teorema negli anelli di serie di potenze per i quali l'anello dei coefficienti sia più generale. Generalizzazioni di questo tipo non si possono però dominare senza indagare più profondamente nella struttura degli anelli l icali e degli anelli di valutazione. Anche qui le ricerche portano anzitutto a generalizzazioni del teorema di preparazione di Weierstrass. Lavori su questa questione trovano le loro origini in KRULL [10], [11] e COHEN [3].

Il problema dell' univoca decomposizione in fattori fornisce un esempio della complicazione che si incontra in quelle ricerche sulle serie di potenze, nelle quali non vale incondizionatamente il teorema di preparazione di Weierstrass. Non si può contestare il significato delle ricerche sugli anelli di serie di potenze con anelli di coefficienti di tipo generale; tale significato risulta tuttavia più evidente nella teoria dei numeri e nell'algebra che nella geometria algebrica, della quale ci occupiamo qui in particolare. Per quanto riguarda quest'ultima, il compito più importante è quello di precisare la struttura degli ideali negli anelli di serie di potenze. Questo compito si divide in due parti : (1) ricerche sulla struttura degli ideali primi, (2) spezzamento di un ideale nelle sue componenti primarie. L'idea conduttrice di queste ricerche è di risolvere $i$ problemi degli ideali di serie di potenze con i mezzi dell'algebra e principalmente mediante la teoria dell'eliminazione. Già la dimostrazione del teorema 17 è un esempio di come le relazioni tra un anello di serie di potenze e un anello di polinomi - nel nostro caso $\mathbb{P}_{k+1}$ e $\mathbb{P} \mathbb{P}_{k}[w]$ - possono essere utilizzate per giungere a teoremi per l'anello di serie di potenze. L'efficacia di questo metodo algebrico proviene dai teoremi di Weierstrass del $\S 6$ e dal teorema seguente, che nei casi più importanti permette di accertarsi della validità delle ipotesi in quei teoremi.

\subsection{Teorema 20.}

Sia ID un corpo con infiniti elementi. Sia a un ideale nell' anello di serie di potenze $\mathbb{D}_{n} x\left(\mathfrak{a} \neq \mathbb{D}_{n} x, \mathfrak{a} \neq(0)\right)$. Allora esiste una $\mathbb{D}$-trasformazione di coor. dinate $(y)_{n}=L(x)_{n}$ e inoltre un numero $k$ che, indicato con $\mathfrak{a}^{*}$ il trastormato di a mediante la $L$, gode delle seguenti proprietà : l'ideale $a^{*}$ contiene. per ogni $i=1,2, \ldots, n-k$ un polinomio $p_{k+i}$ in $y_{k+i}$, che è privilegiato sopra $\mathbb{D}_{k_{+i-1}} y$. $L^{\prime}$ intersezione di $\mathrm{a}^{*}$ con $\mathbb{\$}_{k} y$ è l'ideale nullo.

Supponiamo di aver trovato, per $r \geq 0$, un ideale $\mathfrak{a}^{(r)}$, proveniente da a mediante una $\mathbb{P}$-trasformazione di coordinate:

$$
\left(\xi^{(r)}\right)_{n}=L_{r}(x)_{n}
$$

e che per $i=1,2, \ldots, n-r$ contiene un polinomio $p_{r+i}$ nella $\xi_{r+i}^{(r)}$, che $\dot{\theta}$ privilegiato sopra $\mathbb{P}_{r+i-1} \xi^{(r)}$. Se l'intersenione di $\mathbb{P}_{r} \xi^{(r)}=\mathbb{P}\left[\left[\xi_{2}^{(r)}, \xi_{2}^{(r)}, \ldots, \xi_{r}^{(r)}\right]\right]$ 
con $\mathfrak{a}^{(r)}$ contiene soltanto l'elemento nullo, poniamo, per $k=r: \mathfrak{a}^{*}=\mathfrak{a}^{(r)} \Theta$ $y_{i}=\xi_{i}^{(r)}(i=1,2, \ldots, n)$. Altrimenti tale intersezione contiene una non-unita $f\left(\xi^{(r)}\right)_{r} \neq 0$.

Per il teorema 18 esiste allora una $\mathbb{P}$-trasformazione di coordinate:

$$
\left(\xi^{(r+1)}\right)_{r}=L_{r+1}^{\prime}\left(\xi^{(r)}\right)_{r},
$$

tale che la serie trasformata $f^{*}\left(\xi^{(r+1)}\right)$ r sia privilegiata rispetto a $\xi_{r}^{r+1}$. Per il teorema di preparazione di WEIERSTRASS si ha:

$$
f^{*}=p_{r}\left(\xi_{r}^{(r+1)} \mid\left(\xi^{(r+1)}\right)_{r-1}\right) \cdot e_{r}\left(\xi^{(r+1)}\right)_{r}
$$

ove $e_{r}$ è un' unità e $p$, un polinomio in $\xi_{r}^{(r+1)}$, privilegiato sopra $\mathbb{D}_{r-1} \xi^{(r+1)}$. La trasformazione $L^{\prime}{ }_{r+1}$ opera soltanto sulle $\xi_{1}^{(r)}, \xi_{2}^{(r)}, \ldots, \xi_{r}^{(r)}$. La completiamo ad una $\mathbb{P}$-trasformazione di coordinate $L_{r+1}^{*}$, in tutte le $n$ indeterminate $\xi_{i}(r)$, ponendo:

$$
\xi_{r+i}^{(r+1)}=\xi_{r+i}^{(r)} \quad(i=1,2, \ldots, n-r)
$$

Allora $L_{r+1}$ ¿े definita dalla considerazione simultanea di $L_{r+1}^{*}$ ed $L_{, *}$; dunque :

$$
\left(\xi^{(r+1)}\right)_{n}=L_{r+1}(x)_{n}=L_{r+1}^{*} L_{r}(x)_{n} .
$$

La $L_{r+1}^{*}$ porti $\mathfrak{a}^{(r)}$ in $\mathfrak{a}^{(r+1)}$, cosl che $\mathfrak{a}^{(r+1)}$ proviene da a mediante la $L_{r+1}$. Per le proprietà di $a^{(r)}$ e per la $(4), a^{(r+1)}$ contiene, per ogni $i=2,3, \ldots$, $n-r+1$, un polinomio $p_{r+i-1}$ in $\xi_{r+1-1}^{(r+1)}$, che è privilegiato sopra $\mathbb{D} r+i-2_{2} \xi^{(r+1)}$. Per la (3) anche $p$, appartiene a $a^{(r+1)}$, e tale condizione è percio soddisfatta anche per $i=1$. Si è cosl trovato un ideale $\mathfrak{a}^{(r+1)}$, il quale gode delle pro. prietà volute. Si pud̀ ripetere il procedimento su $\mathbf{a}^{(r+1)}$. Iniziando con $r=0$ e $\mathfrak{a}^{(0)}=\mathfrak{a}$ si ha la possibilità di costruire l'ideale $\mathfrak{a}^{*}$.

Nel teorema 20 si è incontrato il numero $k$, come il numero delle inde. terminate indipendenti di a dopo la $\mathbb{p}$-trasformazione di coordinate $L$. Cosi introdotto, esso potrebbe dipendere da $L$, ma, come si vedrà più tardi, esso coincide con la dimensione di a.

\section{§ 8. - Estensione dagli ideali di polinomi agli ideall di serie di potenze.}

8.1. Il più importante teorema di estensione si enuncia;

Teorema 21, cfr. [4], p. 149 e [7], p. 23.

Sia $R$ un dominio $d$ integrità noetheriano con modulo. Siano 
$R_{l}=R\left[\left[w_{1}, w_{2}, \ldots, w_{l}\right]\right]$ ed $\bar{R}_{l}=R\left[w_{1}, w_{2}, \ldots, w_{l}\right]$. Sia $\bar{w}$ l'ideale $\left(w_{1}, w_{2}, \ldots, w_{l}\right)$ in $\bar{R}_{l}$. Sia $\overline{\mathfrak{q}}$ un ideale primario in $\bar{R}_{l}$ e $\mathfrak{q}=R_{l} \overline{\mathfrak{q}}$ la sua estensione in $R_{l}$, cfr. 1.j. Se l'ideale congiungente $(\overline{\mathfrak{q}}, \overline{\mathbf{w}})$ è diverso da $\bar{R}_{l}$, vale la relazione:

$$
\mathfrak{q} \cap \bar{R}_{l}=\overline{\mathfrak{q}} \text {. }
$$

(a) Sia $\bar{f}_{1}, \bar{f}_{2}, \ldots, \bar{f}_{\text {, }}$ una base di $\bar{q}$. Ogni elemento $f^{\prime}$ di $\mathfrak{q}^{\prime}=\mathfrak{q} \cap \bar{R}_{\boldsymbol{l}}$ possiede una rappresentazione:

$$
f^{\prime}=\mathfrak{t}_{1} \bar{f}_{1}+\mathfrak{t}_{2} \bar{f}_{2}+\ldots+\mathfrak{t}_{r} \bar{f}_{r}
$$

con $\mathfrak{f}_{i} \in R_{\ell}$ per $i=1,2, \ldots, r$. Prefissato ad arbitrio un intero $\rho \geq 0$, vale, per $i=1,2, \ldots, r$, l' ugaaglianza:

$$
\mathfrak{f}_{i}=\bar{p}_{i}+\mathfrak{f}_{i}^{\prime}
$$

dove $\bar{p}_{i}$ è un polinomio in $\bar{R}_{l}$ di grado $\leq \rho$ e $\mathrm{f}_{i}^{\prime}$ una serie di $R_{l}$ con un grado minimo $\geq p+1$. Da ciò segue:

$$
f^{\prime}=\bar{p}_{1} \bar{f}_{1}+\bar{p}_{2} \bar{f}_{2}+\ldots+\bar{p}, \bar{f}_{r}+\mathfrak{f}_{1}^{\prime} \bar{f}_{1}+\mathfrak{f}_{2}^{\prime} \bar{f}_{2}+\ldots+\mathfrak{f}_{r}^{\prime} \bar{f}_{n}
$$

Ora $f^{*}=f^{\prime}-\vec{p}_{1} \bar{f}_{1}-\bar{p}_{2} \bar{f}_{2}-\ldots-\bar{p}_{r} \bar{f}_{r}$ è un polinomio di $\bar{R}_{l}$ con grado minimo $\geq p+1$ ed appartiene percio a $\bar{W}^{\rho}$. Quindi è $f^{\prime} \in\left(\overline{\mathrm{q}} \cdot \bar{W}^{\circ}\right)$ per ogni $\rho \geq 0$. L'intersezione di tutti $i(\overline{\mathfrak{q}}, \overline{\mathcal{W}})$ per $\rho \geq 0$ è un ideale $\bar{\delta}$ di $\bar{R}_{l}$, ed è $\mathfrak{q}^{\prime} \subset \bar{\delta}$.

(b) Consideriamo ora l'ideale $(\overline{\mathfrak{w}} \cdot \overline{\boldsymbol{\delta}}, \overline{\mathfrak{q}})$ di $\bar{R}_{l}$. Da $\overline{\mathfrak{w}} \cdot \bar{\delta} \subset \overline{\boldsymbol{\delta}}$ e $\overline{\mathfrak{q}} \subset \bar{\delta}$ segue $(\overline{\mathfrak{W}} \cdot \bar{\delta}, \overline{\mathfrak{q}}) \subset \bar{\delta}$. Mostreremo che in questa relazione vale il segno di nguaglianza. A questo scopo sia:

$$
(\overline{\mathbf{w}} \cdot \overline{\mathbf{\delta}}, \overline{\mathfrak{q}})=\overline{\mathfrak{q}}_{\mathrm{i}} \cap \ldots \cap \overline{\mathfrak{q}}_{\mathrm{s}}
$$

una rappresentazione mediante componenti primarie. Per $\lambda=1,2, \ldots, s$ l'ideale primo $\overline{\mathfrak{p}}_{\lambda}$ appartenga a $\overline{\mathbf{q}}_{\lambda}$. Distinguiamo due easi :

(1) $w$ non giaccia in $\overline{\mathfrak{p}}_{\lambda}$. Poichè $\overline{\mathbf{w}} \cdot \bar{\delta} \subset \overline{\mathbf{q}}_{\lambda}$, ne segue, per la proprieta fondamentale degli ideali primari, che $\bar{\delta} \subset \overline{\mathfrak{q}}_{\lambda}$.

(2) $\overline{\mathbf{w}} \subset \overline{\mathbf{p}}_{\lambda}$. Per un esponente $\rho$ abbastanza grande vale allora $\overline{\mathbf{w}}_{\rho} \subset \overline{\mathbf{q}}_{\lambda}$, e per la definizione di $\bar{\delta}$ è $\bar{\delta} \subset(\bar{W}, \overline{\mathfrak{q}})$. Poichè è anche $\mathfrak{q} \subset(\overline{\mathfrak{W}} \cdot \overline{\boldsymbol{\delta}}, \overline{\mathfrak{q}}) \subset \mathfrak{q}_{\lambda}$, otteniamo di nuovo $\bar{\delta} \subset \overline{q_{\lambda}}$. Questa relazione è quindi vera per ogni $\lambda$. Di qui segue:

$$
\bar{\delta} \subset \overline{\mathfrak{q}}_{1} \cap \overline{\mathfrak{q}}_{2} \cap \ldots \cap \overline{\mathfrak{q}}_{s}=(\overline{\mathfrak{w}} \cdot \overline{\boldsymbol{d}}, \overline{\mathfrak{q}})
$$

e come affermato :

$$
\overline{\boldsymbol{\delta}}=(\overline{\mathbf{w}} \cdot \overline{\boldsymbol{\delta}}, \overline{\mathbf{q}})
$$



tazioni :

(c) Sia $\bar{d}_{1}, \bar{d}_{2}, \ldots, \bar{d}_{t}$ una base per $\bar{\delta}$. Dalla (1) seguono le rappresen.

$$
\bar{d}_{i}=\sum_{j=1}^{t} \bar{\omega}_{i_{j}} \bar{d}_{j}+\bar{q}_{i}, \quad i=1,2, \ldots, t,
$$

con $\bar{\omega}_{i j}$ elementi di $\bar{w}$ e $\bar{q}_{i}$ di $\overrightarrow{\mathfrak{q}}$. Vale allora:

$$
\sum_{j=1}^{t}\left(\delta_{i j}-\bar{\omega}_{i j}\right) \bar{d}_{j}=\bar{q}_{i}, \quad i=1,2, \ldots, t,
$$

con $\delta_{i j}=1$ per $i=j$ e $\delta_{i j}=0$ per $i \neq j$. Indicando con $\Delta$ il determinante $\left\|\delta_{i j}-\bar{\omega}_{i j}\right\|$, si ha:

$$
\Delta \bar{d}_{i} \in \overline{\mathfrak{q}}, \quad i=1,2, \ldots, t .
$$

D'altra parte è $\Delta=1+\bar{\omega}$ con $\bar{\omega} \in \bar{W}$, e perciò anche $\Delta^{n}$ è della stessa forma, per ogni esponente $n \geq 1: \Delta^{n}=1+\bar{\omega}_{n}, \bar{\omega}_{n} \in \overline{\mathcal{W}}$. Se ora $\Delta^{n}$ fosse in $\overline{\mathbf{q}}$, sarebbe $1 \in \overline{\mathfrak{q}}, \overline{\mathfrak{W}})$, e $(\overline{\mathfrak{q}}, \overline{\mathfrak{w}})=\overline{R_{l}}$, cosa che noi abbiamo escluso. Poichè $\overline{\mathfrak{q}} \grave{e}$ primario e non contiene alcuna potenza di $\Delta$, deve perciò essere $d_{i} \in \overline{\mathfrak{q}}$ per $i=1,2, \ldots, t$. E dunque $\bar{\delta} \subset \overline{\mathfrak{q}}$. Essendo in $(a)$ stato dimostrato che $\overline{\mathrm{q}} \subset \mathfrak{q} \subset \overline{\mathrm{d}}$, ne segue l'affermazione $\mathfrak{q}^{\prime}=\overline{\mathfrak{q}}$.

8.2. Il teorema 21 si può generalizzare al caso di ideali arbitrari. A questo scopo dimostriamo dapprima, adoperando le stesse notazioni di 8.1., un complemento $(a)$ ed un lemma $(b)$.

(a) Sia $\overline{\mathbf{a}}$ un ideale in $\bar{R}_{l}$ con $(\overline{\mathfrak{a}}, \overline{\mathfrak{w}})=\bar{R}_{l}$. Allora ò l'estensione $\mathfrak{a}=R_{l} \cdot \overline{\mathfrak{a}}=R_{l}$.

Dall'ipotesi segue per 1 la rappresentazione $1=\bar{a}+\bar{\omega}$ con $\bar{a} \in \overline{\mathfrak{a}}$ e $\bar{\omega} \in \overline{\mathfrak{w}}$. Per il teorema $4 \vec{a}$ è una unità in $R_{l}$ e perciò $\mathfrak{a}=R_{l}$.

(b) Siano $\overline{\mathfrak{a}}$ e $\overline{\mathfrak{b}}$ ideali di $\bar{R}_{l}$, tali che $(\overline{\mathfrak{a}}, \overline{\mathfrak{w}})=\bar{R}_{l}$ e $(\overline{\mathfrak{b}}, \overline{\mathfrak{w}})=\bar{R}_{l}$. Allora $\dot{\theta}$ anche $(\overline{\mathfrak{a}} \cap \overline{\mathfrak{b}}), \overline{\mathbf{w}})=\bar{R}_{l}$.

Dalle ipotesi seguono per 1 le rappresentazioni $1=\bar{a}+\bar{\omega}_{1}, \quad 1=\bar{b}+\bar{\omega}_{2}$, con $\bar{a} \in \overline{\mathfrak{a}}, \bar{b} \in \mathfrak{b}$ e $\bar{\omega}_{1}, \bar{\omega}_{2} \in \overline{\mathfrak{w}}$. La moliplioazione di queste due formule porge $1=\bar{a} \bar{b}+\bar{\omega}$ con $\bar{\omega} \in \bar{w}$. Da $\bar{a} \cdot b \in \overline{\mathbf{a}} \cap \overline{\mathbf{b}}$ segue quindi l' affermazione.

8.3. Thorema 22, efr. [4], p. 149 .

Sia $R$ un dominio d'integrità noetheriano con modulo. Sia $R_{l}=R\left[\left[w_{1}, w_{2}, \ldots, w_{l}\right]\right]$ e $\bar{R}_{l}=R\left[w_{1}, w_{2}, \ldots, w_{l}\right]$. Sia $\bar{w}$ l'ideale $\left(w_{1}, w_{2}, \ldots, w_{l}\right)$ in $\bar{R}_{l}$. Un ideale $\overline{\mathrm{a}}$ in $R_{l}$ è intersezione degli ideali $\overline{\mathrm{b}}$ e $\overline{\mathrm{c}}$, dove $\overline{\mathrm{b}}$ non contiene alcuna componente primaria $\overline{\mathfrak{q}}$ con $(\overline{\mathfrak{q}}, \overline{\mathfrak{w}})=\bar{R}_{l}$ e $(\overline{\mathcal{c}}, \overline{\mathbf{w}})=\bar{R}_{l}$. Essendo 
a l'ideale esteso $R_{l} \cdot \overline{\mathfrak{a}}$ di $\overline{\mathfrak{a}}$ in $R_{l}$, vale la relazione:

$$
\mathfrak{a} \cap \bar{R}_{l}=\overline{\mathfrak{b}} \text {. }
$$

(a) Sia $\overline{\mathfrak{a}}=\overline{\mathfrak{q}}_{1} \cap \overrightarrow{\mathfrak{q}}_{2} \cap \ldots \cap \overline{\mathfrak{q}}_{r}$ una rappresentazione con componenti primarie di $\overline{\mathfrak{a}}$. Per gli ideali primari $\overline{\mathfrak{q}}_{i_{1}}, \overline{\mathfrak{q}}_{i_{2}}, \ldots, \overline{\mathfrak{q}}_{i_{s}}$ sia $\left(\overline{\mathfrak{q}}_{i_{\lambda}}, \overline{\mathfrak{w}}\right) \neq R_{l}$, mentre per i rimanenti $\overline{\mathfrak{q}}_{j_{1}}, \overline{\mathfrak{q}}_{j_{2}}, \ldots, \overline{\mathfrak{q}}_{j_{t}}$ valga $:\left(\overline{\mathfrak{q}}_{j_{\mu}}, \overline{\mathbb{W}}\right)=\bar{R}_{l}$. Posto:

$$
\overline{\mathfrak{b}}=\overline{\mathfrak{q}}_{i_{1}} \cap \overline{\mathfrak{q}}_{i_{2}} \cap \ldots \cap \overline{\mathfrak{q}}_{i_{s}} \quad \text { e } \quad \overline{\mathbf{c}}=\overline{\mathfrak{q}}_{j_{1}} \cap \overline{\mathfrak{q}}_{j_{2}} \cap \ldots \cap \overline{\mathfrak{q}}_{j_{t}}
$$

è $\overline{\mathbf{a}}=\overline{\mathbf{b}} \cap \overline{\mathbf{c}}$, e, per la 8.2. (b), $(\overline{\mathbf{c}}, \overline{\mathbf{w}})=\bar{R}_{l}$. Con ciò è stabilita la voluta rappresentazione di $\overline{\mathfrak{a}}$.

(b) Dalla 1.3. (c) segue:

$$
\mathfrak{a}=R_{l} \cdot \overline{\mathfrak{a}} \subset\left(R_{l} \cdot \overline{\mathfrak{b}}\right) \cap\left(R_{l} \cdot \overline{\mathrm{c}}\right)=R_{l} \cdot \overline{\mathfrak{b}}
$$

poichè per la 8.2. (a) è $R_{l} \cdot \overline{\mathrm{c}}=R_{l}$. Posto $\mathfrak{q}_{i_{\lambda}}=R_{l} \cdot \overline{\mathfrak{q}}_{i_{\lambda}}$, per $\lambda=1,2 \ldots, s$, per il teorema 21 è

$$
\mathfrak{q}_{i_{\lambda}} \cap \bar{R}_{l}=\overline{\mathfrak{q}}_{i_{\lambda}}
$$

Inoltre dalla 1.3. (c) segue:

$$
R_{l} \cdot \overline{\mathfrak{b}} \subset \mathfrak{q}_{i_{1}} \cap \mathfrak{q}_{i_{2}} \cap \ldots \cap \mathfrak{q}_{i_{\varepsilon}},
$$

e anche:

$$
\mathfrak{a} \subset \mathfrak{q}_{i_{1}} \cap \mathfrak{q}_{i_{2}} \cap \ldots \cap \mathfrak{q}_{i_{\delta}}
$$

Da cio segue, per le 1.3. (a) e (1):

$$
\mathfrak{a} \cap \bar{R}_{l} \subset \overline{\mathfrak{b}}
$$

(c) D'altra parte è $\bar{b} \cdot \bar{c} \subset \overline{\mathfrak{a}}$ e per la 1.3. (c):

$$
R_{l} \cdot(\overline{\mathfrak{b}} \cdot \overline{\mathfrak{c}})=\left(R_{l} \cdot \overline{\mathfrak{b}}\right) \cdot\left(R_{l} \cdot \overline{\mathfrak{c}}\right)=R_{l} \cdot \overline{\mathfrak{b}} \subset \mathfrak{a} .
$$

Facendo l'intersezione con $\bar{R}_{l}$ si ha naturalmente $\overline{\mathfrak{b}} \subset\left(R_{l} \cdot \overline{\mathfrak{b}}\right) \cap \bar{R}_{l}$. Da ciò segue $\bar{b} \subset \mathfrak{a} \cap \bar{R}_{l}$. Questa relazione e la (2) porgono l'affermazione.

8.4. In un importante caso particolare si dimostra, a complemento del teorema 21, che l'estensione di un ideale primario è primario. 


\section{Teorema 23.}

Sia $\mathbb{P}$ un dominio d'integritò noetheriano con modulo, e $\mathbb{P}_{k+i}$ l'anello delle serie di potenze $\mathbb{P}\left[\left[z_{1}, z_{2}, \ldots, z_{k}, w_{1}, w_{2}, \ldots, w_{i}\right]\right], i \geq 1, \mathbb{P}_{k}=\mathbb{P}_{k} z$. Sia inoltre $\overline{\mathbb{D}}_{k, i}$ l'anello di polinomi $\mathbb{P}_{k}\left[w_{1}, w_{2}, \ldots, w_{i}\right]$. Sia $\overline{\mathfrak{q}}$ un ideale primario in $\overline{\mathbb{D}}_{k, l}$ con le seguenti proprietà : (a) per ogni valore $i=1,2, \ldots, l, \overline{\mathfrak{q}}$ contenga un polinomio $\bar{p}_{i}$ dell' anello $\overline{\mathbb{D}}_{k, i}$, che come polinomio in $w_{i}$ sia privilegiato sopra $\mathbb{P}_{k+i-1}$. (b) essendo $\overline{\mathbb{W}}$ l'ideale $\left(w_{1}, w_{2}, \ldots, w_{l}\right)$ in $\overline{\mathbb{P}}_{k, l}$, sia $(\overline{\mathfrak{q}}, \overline{\mathbf{W}}) \neq \overline{\mathbb{P}}_{k, l}$. Allora l'i leale esteso $\mathfrak{q}=\mathbb{D}_{k+l} \cdot \overline{\mathfrak{q}}$ di $\overline{\mathfrak{q}}$ in $\mathbb{\mathfrak { P }}_{k+l}$ è un ideale primario in $\mathbb{\mathbb { P }}_{k+l}$ e vale la relazione:

$$
\overline{\mathfrak{q}}=\overline{\mathbb{p}}_{k, l} \cap \mathfrak{q} .
$$

La formula $8(3)$ è una conseguenza dell'ipotesi (b) e del teorema 21. Sia $f$ una serie in $\mathfrak{q}$ ed $f=g \cdot h$ con $g, h \in \mathbb{P}_{k+l} ; h$ non appartenga a $\mathfrak{q}$. Dobbiamo dimostrare che una potenza di $g$ appartiene a $\mathfrak{q}$. Per il teorema $13 \grave{e}: g=q_{1}+\bar{g}, h=q_{2}+\bar{h}$ con $q_{1}, q_{2} \in \mathfrak{q}$ e $\bar{g}, \bar{h} \in \overline{\mathbb{D}}_{k, i}$, da cui segue :

$$
f=g \cdot h=q_{1} \cdot q_{2}+q_{1} \cdot \bar{h}+q_{2} \cdot \bar{g}+\bar{g} \cdot \bar{h}
$$

Posto $\bar{f}=f-q_{1} q_{2}-q_{1} \bar{h}-q_{2} \bar{g}$, si ha $\bar{f}=\bar{g} \cdot \bar{h} \in \mathfrak{q} \cap \overline{\mathbb{D}}_{k, l}=\overline{\mathfrak{q}}$. Inoltre $\bar{h}$ non $\dot{\theta}$ un elemento di $\overline{\mathfrak{q}}$, poichè altrimenti $h$ dovrebbe appartenere a $\mathfrak{q}$. Poiche $\overline{\mathfrak{q}} \dot{\theta}$ un ideale primario in $\overline{\mathbb{D}}_{k, l}$, una potenza $\bar{g}^{p}$ appartiene a $\overline{\mathfrak{q}} ;$ e da $\bar{g}^{p}=\left(g-q_{1}\right) p \in \overline{\mathfrak{q}}$ segue ora $g^{\circ} \in \mathfrak{q} \cdot \mathfrak{q}$ è dunque un ideale primario in $\mathbb{P}_{k+l}$.

Corollario 1.

Se $\mathfrak{q}$ è un ideale primo in $\overline{\mathbb{D}}_{k, l}$, nelle ipotesi del teorema 23, allora $\mathfrak{q}=\mathbb{P}_{k+l} \cdot \overline{\mathfrak{q}} \dot{\mathrm{e}}$ un ideale primo in $\mathbb{\mathbb { P }}_{k+l}$.

Nella precedente dimostrazione ora è $\rho=1$. Dalle definizioni segue il:

Conollario 2.

Se, nelle ipotesi del teorema 23 l'ideale primo $\overline{\mathfrak{p}}$ appartiene all'ideale primario $\overline{\mathfrak{q}}, \mathfrak{q}=\mathbb{p}_{k+l} \cdot \overline{\mathfrak{q}}$ 㝴 un ideale primario dell'ideale primo $\mathfrak{p}=\mathbb{p}_{k+l} \cdot \overline{\mathfrak{p}}$.

\section{§ 9. - La struttura di un ideale primo.}

9.1. In conformità del programma di cui al n. 7.5. esaminiamo ormai più esattamente la costruzione degli ideali primi dell'anello di serie di potenze $\mathbb{P}$.. . A fondamento di tutte queste considerazioni poniamo come anello dei coefficienti $\mathbb{D}$ un corpo con infiniti elementi. 
Teorema 24.

Sia $\mathbb{P}$ un corpo con infiniti elementi; $\mathfrak{p}$ un ideale primo nell'anello di serie di potenze $\mathbb{P}_{n}(\mathfrak{p} \neq \mathbb{P}$.). Esiste allora una $\mathbb{P}$-trasformazione di coordinate $(y)_{n}=L(x)_{n}$, tale che l'ideale trasformato possiede una base nell'anello di polinomi $\quad \overline{\mathbb{D}}_{k, n-k}=\mathbb{P}\left[\left[y_{1}, y_{2}, \ldots, y_{k}\right]\right]\left[y_{k+1}, y_{k+2}, \ldots, y_{n}\right]$ e vale la relazione $\mathbb{1}_{k} y \cap \mathfrak{p}=(0)$. Questa base contiene, per $i=1,2, \ldots, n-k$, un polinomio irriducibile $\vec{p}_{k+i}$ in $y_{k+1}, y_{k+2}, \ldots, y_{k+i}$ che come polinomio in $y_{k+i} \grave{e}$ privilegiato sopra $\mathbb{D}_{k_{+i-1}} y$. In queste condizioni noi parliamo di una base di polinomi in $\mathbb{\mathbb { P }}_{k, n-k}$. Il numero $k$ è uguale alla dimensione di $\mathfrak{p}$, cfr. 4.3. Essendo $f$ una arbitraria serie di $\mathbb{P}_{n}$, esiste un elemento $p$ di $\mathfrak{p}$ e un polinomio $\bar{f} d i \overline{\mathbb{D}}_{k, n-k}$, tali che:

$$
f=\bar{f}+p
$$

Indicando con $\overline{\mathfrak{p}}$ l'ideale $\mathfrak{p} \cap \overline{\mathbb{p}}_{k, n-k}, \overline{\mathfrak{p}}$ è un ideale primo in $\overline{\mathbb{D}}_{k, n-k}$. Viceversa $\mathfrak{p}$ è l'estensione di $\overline{\mathfrak{p}}$ in $\mathbb{D}_{n}$. L' anello delle classi reslo $\mathbb{D}_{n} / \mathfrak{p}$ è isomorfo all' anello algebrico delle classi resto $\overline{\mathbb{p}}_{k, n-k} / \overline{\mathbf{p}}$.

I precedenti risultati sulla base di polinomi sono conseguenza dei teoremi 20 e 14. La rappresentazione $9(1)$ segue del teorema 13. Le relazioni fra $\mathfrak{p}$ e $\overline{\mathfrak{p}}$ seguono dal teorema 23 . L'isomorfismo tra l'anello $\mathbb{P}_{n} / \mathfrak{p}$ e l'anello $\overline{\mathbb{D}}_{k, n-k} / \bar{p}$ sussiste: in forza della formula $9(1)$, cosicchè la classe resto di $\bar{f}$ in $\overline{\mathbb{D}}_{k, n-k} / \overline{\mathfrak{p}}$ è l'immagine della classe resto di $f$ in $\mathbb{P}_{n} / \mathfrak{p}$. L'affermazione circa il valore di $k$ potrà essere dimostrata soltanto più tardi, in 9.7.

9.2. Come segue dal teorema 23, vale una inversione del teorema 24 , nel senso seguente:

\section{Corollario 1.}

Sia $\overline{\mathfrak{p}}$ un ideale primo dell'anello di polinomi $\overline{\mathbb{D}}_{k, n-k}$, e sia $\overline{\mathfrak{p}}, y_{k+1}, y_{k+2}$, $\left.\ldots, y_{n}\right) \neq \overline{\mathbb{D}}_{k, n-k}$. In $\overline{\mathbf{p}}$ siano contenuti dei polinomi $\bar{p}_{k+i}$ con le proprieta descritte nel teorema 24 . Allora l'ideale esteso $\mathfrak{p}$ di $\bar{p}$ in $\mathbb{P}_{n}$ è primo, ed è $\mathfrak{p} \cap \overline{\mathbb{P}}_{k, n-k i}=\overline{\mathbf{p}}$.

L'altima asserzione del teorema 24 si rivela un importante mezzo per dedarre ulteriori proprietà dell'ideale primo $\mathfrak{p}$; essa infatti riconduce l'analisi di $\mathbb{P}_{n} / \mathfrak{p}$ al problema algebrico dello studio di un'estensione algebrica; precisamente vale il:

\section{Corollario 2.}

L'anello delle classi resto $\mathbb{D}_{n} / \mathfrak{p}$ è isomorfo ad una estensione algebrica intera di un anello di serie di potenze $\mathbb{D}_{k} \operatorname{con} k<n$. 
Indicando infatti, per $i=1,2, \ldots, n-k$. con $\eta_{k+i}$ la classe resto di $y_{k+i} \bmod \overline{\mathfrak{p}}, \mathbb{P}_{n} / \mathfrak{p}$ \& isomorfo all'anello $\mathbb{P}_{k}\left[\eta_{k+1}, \eta_{k+2}, \ldots, \eta_{n}\right]$ estensione algebrica di $\mathbb{D}_{k}$, e questo è algebrico intero sopra $\mathbb{D}_{k}$, perchè $\overline{\mathfrak{p}}$ contiene i polinomi $\bar{p}_{k+i}$.

Cónollakio 3.

Sia $f$ una serie di $\mathbb{P}_{n}$. Se $f$ non appartiene a $p$, esiste un polinomio di $f$ :

$$
f^{m}+c_{1}(y)_{k} f^{m-1}+\ldots+c_{m-1}(y)_{k} f+c_{m}(y)_{k}
$$

con coefficienti in $\mathbb{D}_{k}$ e $c_{m} \neq 0$ che appartiene a $\mathfrak{p}$.

Essendo il polinomio $\bar{f}$ definito secondo la $9(1), \vec{f}$ individua nna classe resto $\bar{\varphi}$ di $\overline{\mathbb{P}}_{k, n-k} \overline{\mathbf{p}}$. Ora $\bar{\varphi} \dot{\rho}$ algebrico intero sopra $\mathbb{P}_{k}$ e soddisfa perciò un'equazione algebrica irriducibile:

$$
\bar{\varphi}^{m}+\gamma_{1} \bar{\rho}^{m-1}+\ldots,+\gamma_{m}=0 .
$$

dove le $\gamma_{i}$ sono classi resto di elementi di $\mathbb{D}_{k}$ in $\overline{\mathbb{P}}_{k, n-k}$ e $\gamma_{m} \neq 0$. E queste classi resto possiamo senz'altro identificarle con elementi $c_{i}(y)_{k}$ di $\mathbb{D}_{k}$. Ritor. nando dalle classi resto all'anello $\overline{\mathbb{D}}_{k, n-k}$ questa equazione significa:

$$
\bar{f}^{m}+c_{1}(y)_{k} \bar{f}^{m-1}+\ldots+c_{m}(y)_{k} \equiv 0 \bmod \overline{\mathfrak{p}} .
$$

Sostituendo qui $\bar{f}$ con $f-p$, si ottiene la proposizione voluta 9(2).

La serie $c_{n} \in \mathbb{D}_{k}$ si chiama *Norma》 di $f$ rispetto a p. Questa norma non è una unità di $\mathbb{P}_{k}$, se $f$ non è una unità di $\mathbb{P}_{n}$.

9.3, Per le estensioni algebriche finite si dimostra nell'algebra il teorema dell' elemento primitivo (cfr. [4], pag. 29):

Ognj estensione algebrica finita $\mathfrak{U}=K\left(u_{1}, u_{2}, \ldots, u_{n}\right)$ di un corpo perfetto $K$ con infiniti elementi può essere generata con l'aggiunta di un cosidetto elemento primitivo $w$. Si ottiene $w$ nella forma:

$$
w=\alpha_{1} u_{1}+\alpha_{2} u_{2}+\ldots+\alpha_{n} u_{n}
$$

con coefficienti $\alpha_{i}$ in un arbitrario sottocorpo $A$ di $K$ con infiniti elementi.

Se $K$ e il corpo quoziente del dominio d'integrita $R$ ed $R$ è integralmente chiuso in $K$, si dimostra inoltre, ofr. [4], pag. 132: Se $u_{1}, u_{2}, \ldots, u_{n}$ sono interi algebrici sopra $R$, allora $w$ può essere scelto intero algebrico sopra $R$. Se $w$ soddisfa l'equazione irriducibile $p(w)=0, p \in R[w]$, ogni elemento intero algebrico $g$ di $\mathbb{U}$ è rappresentabile nella forma :

$$
g=G(w) / D
$$

dove $G \in R[w]$ e $D$ indica il discriminante di $p$. 
Questi teoremi vanno applicati con $R=\mathbb{D}_{k}$ e $A=\mathbb{D}$. Che $\mathbb{D}_{k}$ sia integra]mente chiuso nel suo corpo quoziente, si dimostra esattamente come per gli anelli di polinomi. Questa proprietà segue facilmente dal fatto che $\mathbb{P}_{k}$ è un dominio d'integrità con decomposizione univoca in fattori. efr. [7]. prg. $76 \mathrm{e}$ teorema 19. In Itre si dovrebbe supporre che $\mathbb{D}_{x}$ sia perfetto. Qunndo la caratteristica di $\mathbb{P}$ è un numero primo, questa proprietá, com'è noto, non segue dall'essere $\mathbb{D}$ perfetto.

Esauriamo dapprina il caso di caratteristica 0.

\subsection{TEOREMA 25.}

Sia $\mathbb{P}$ un corpo di caratteristica 0 . Sia p un ideale primo nell'anello delle serie di potenze $\mathbb{P}_{n}\left(\mathfrak{p} \neq \mathbb{D}_{n}\right)$. Esiste allora una $\mathbb{P}$-trasformazione di coordinate $(z)_{n}=L(x)_{n}$, tale che l'ideale trasformato contiene elementi del tipo seguente:

(a) Per $z_{k+1}$ un polinomio irriducibile $F\left(z_{k+1} !(z)_{k}\right)$ dell'anello $\mathbb{P}_{k}\left[z_{k+1}\right]$. che è privilegiato sopra $\mathbb{\mathbb { P }}_{k}$.

(b) Per $i=2,3, \ldots, n-k$ un polinomio lineare in $z_{k+i}$ della forma:

$$
D(z)_{k} \cdot z_{k+i}-G_{k+i}\left(z_{k+1} ;(z)_{k}\right)
$$

dove $G_{k+i}$ è un polinomio dell' anello $\mathbb{1}_{k}\left[z_{k+1}\right]$ e $D$ il discriminante di $F$.

Per la dimostrazione stabiliamo anzitutto, dopo una $\mathbb{P}$-trasformazione di coordinate $\left(y_{n}=L_{1}(x)_{n}\right.$, una base dei polinomi di $\mathfrak{p}$, appartenente all'anello $\overline{\mathbb{D}}_{k, n-k}$, ofr. Teorema 24 , e sia $\overline{\mathfrak{p}}=\mathfrak{p} \cap \overline{\mathbb{D}}_{k, n-k}$. Dopo ciò costruiamo un elemento primitivo come combinazione lineare:

$$
z_{k+1}=\beta_{k+1} y_{k+1}+\beta_{k+2} y_{k+2}+\ldots+\beta_{n} y_{n}
$$

con $\beta_{k+i} \in \mathbb{P}$. Sia $\eta_{k+i}$ la classe resto di $y_{k+i} \bmod \bar{p}$ e

$$
\xi_{k+1}=\beta_{k+1} \eta_{k+1}+\beta_{k+2} \eta_{k+2}+\ldots+\beta_{n} \eta_{n} .
$$

Sia poi $F$ il polinomio irriducibile appartenente a $\mathbb{D}_{k}\left[z_{k+1}\right]$ con la radice $\xi_{k+1}$. Allora sussistono le rappresentazioni :

$$
\eta_{k+i}=G_{k+i}\left(\xi_{k+1}\right) / D
$$

con $G_{k+i} \in \mathbb{P}_{k}\left[z_{k+i}\right]$, essendo $D$ il discriminante di $F$.

Queste equazioni dicono pertanto che le quantità $D y_{k+i} G_{k+i}$ appartengono a $\bar{p}$ e perciò a $\mathfrak{p}$. Senza venir meno alla generalita possiamo supporre $\beta_{k+1} \neq 0$; ciò si può infatti ottenere con una opportuna numerazione d lle $y_{k+i}$. Definiamo allora mediante la $9(4)$ e mediante le $z_{i}=y_{i}$ per $i=1,2, \ldots$, $k, k+2, \ldots, n$ una $\mathbb{P}$-trasformazione di coordinaie $L_{2}$. Con $(z)_{n}=L_{2} \cdot L_{1}(x)_{n}$ si soddisfa allora l'enunciato del teorema. 
9.5. Nel caso della caratteristica $p>0$ si puo procedere analogamente al caso algebrico. cfr. [12]. pag. 74. Il fondamento di questo trasporto consiste nel seguente teorema:

Sia $\mathbb{P}$ un corpo perfetto di caratteristica $p$, e sia $f$ una serie dell' anello delle serie di potenze $\mathbb{P}_{n}$. In $f$ facciamo la sostituzione $x_{i}=\xi_{i}^{p l}$ per $i=1,2$, $\ldots, n$; si può allora dalla serie trasformata estrarre la radice $p^{l}$.

Se infatti $f=\Sigma a_{v_{1} v_{2} \ldots v_{n}} x_{1}^{\nu_{1}} x_{2}^{\nu_{2}} \ldots x_{n}^{v_{n}}$, sarà

$$
(f)^{1 / p l}=\Sigma\left(a_{v_{1} \nu_{2} \ldots \nu_{n}}\right)^{1 / p l \xi_{1}^{\nu_{1}}} \cdot \xi_{2}^{v_{2}} \ldots \xi_{n^{n}}^{v^{n}}
$$

Nella 9(4) considerando $\beta_{k+1}, \beta_{k+2}, \ldots, \beta_{n}$ come indeterminate, $\xi_{k+1}$ e radice di un polinomio irriducibile $\Phi\left(z_{k+1} /(y)_{k}, \beta_{k+1}, \beta_{k+2}, \ldots, \beta_{n}\right)$ dell' anello $\mathbb{P}_{k}\left[\beta_{k+1}, \beta_{k+2}, \ldots, \beta_{n}, z_{k+1}\right]$. Se $\partial \Phi / z_{z_{+1}} \neq 0$, si possono applicare le ordinarie considerazioni che conducono alla costruzione di un elemento primitivo. In questo caso vale il teorema 25. Essendo $\partial \Phi / \partial z_{k+1}=0$, esiste una potenza massima $z_{k+1}^{p^{l}}$, tale che $\Phi$ é un polinomio di $z_{k+1}^{p^{l}}$. Allora si può dimostrare che $\Phi$ è anche polinomio di $\beta_{k+1}^{p^{l}}, \beta_{k+2}^{p^{l}}, \ldots, \beta_{n}^{p}$. Introduciamo in $\Phi$ :

$$
y_{i}=Y_{i}^{p l}, \quad i=1,2, \ldots, k .
$$

Per mezzo del nostro lemma si può dimostrare facilmente che $\Phi$ è potenza $p^{\ell}$ di un polinomio irriducibile $\Psi\left(z_{k+1}(Y)_{k}, \beta_{k+1}, \beta_{k+2}, \ldots, \beta_{n}\right)$ dell' anello $\mathbb{P}_{k}^{\prime}\left[\beta_{k+1}, \beta_{k+2}, \ldots, \beta_{n}, z_{k+1}\right]$, con $\mathbb{P}_{k}^{\prime}=\mathbb{P}_{k} Y$. Definiamo l'ideale $\mathfrak{p}^{\prime}$ dell' anello $\mathbb{D}_{k, n-k}^{\prime}=\mathbb{P}_{k}^{\prime}\left[y_{k+1}, y_{k+2}, \ldots, y_{n}\right]$ mediante la seguente posizione: un polinomio $\rho$ di quest'anello appartiene a $\mathfrak{p}^{\prime}$, se $\wp^{p l} \in \mathfrak{p}$. Si dimostra facilmente che $\mathfrak{p}^{\prime} \grave{e}$ primo e che $\mathfrak{p}^{\prime} \cap \mathbb{D}_{k}^{\prime}=(0)$. Indicando con il simbolo $H_{k+i}$ la classe resto di $y_{k+i} \bmod \mathfrak{p}^{\prime}$ in $\overline{\mathbb{P}}_{k, n-k}^{\prime}$ è

$$
z_{k+1}=\beta_{k+1} H_{k+1}+\beta_{k+2} H_{k+2}+\ldots+\beta_{n} H_{n}
$$

una radice di $\Psi$. Inoltre è ora $\partial \Psi / \partial z_{k+1} \neq 0$, cosicchè si può applicare a $\mathfrak{p}^{\prime}$ il teorema 25. Con opportuna specializzazione di $\beta_{k+1}, \beta_{k+2}, \ldots, \beta_{n}$ in $\mathbb{P}$ vale quanto segue: $(a)$ esiste in $\mathfrak{p}^{\prime}$ un polinomio irriducibile $F^{\prime}\left(z_{k+1} /(Y)_{k}\right)$ dell'anello $\mathbb{P}_{k}^{\prime}\left[z_{k+1}\right]$ che è privilegiato sopra $\mathbb{P}_{k}^{\prime}$. (b) In $\mathfrak{p}^{\prime}$ esiste, per $i=2,3, \ldots$, $n-k$, un polinomio lineare $D^{\prime}(Y)_{k} y_{k+i}-G_{k+i}^{\prime}\left(z_{k+1} ;(Y)_{k}\right)$ con $G^{\prime} k_{+i}$ in $\mathbb{P}_{k}^{\prime}\left[z_{k+1}\right]$ e il discriminante $D^{\prime}$ di $F^{\prime}$. Per la definizione di $\mathfrak{p}^{\prime}, \mathfrak{p}$ contiene $(a)$ un polinomio $\left(F^{\prime}\right)^{p l}=F^{p}\left(z_{k_{+1}} /(y)_{k}\right)$ dell'anello $\mathbb{P}_{k}\left[z_{k+1}^{p^{l}}\right],(b)$ per $i=2,3, \ldots, n-k$ un polinomio $D(y)_{k} \cdot y_{k-i}^{p^{l}}-G_{k+i}\left(z_{k+1}:(y)_{k}\right)$ con $G_{k+i} \in \mathbb{P}_{k}\left[z_{k+1}^{p^{l}}\right]$. Pertanto $D \in \mathbb{P}_{k}$ non è piu il discriminante di $F$. Definendo allora le $\mathbb{P}$-trasformazioni di coordinate $L_{2}$ ed $L$ come prima, vale in luogo del teorema 25 il: 


\section{Teorema 26.}

Sia $\mathbb{P}$ un corpo perfetto di caratteristica $p$ con infiniti elementi. Nell'anello delle serie di potenze $\mathbb{D}_{n} x$ sia $\mathfrak{p}$ un ideale primo $\left(\mathfrak{p} \neq \mathbb{D}_{n} x\right)$. Esiste allora una D-trasformazione di coordinate $(z)_{n}=L(x)_{n}$ tale che l'ideale trasformato, per una opportuna scelta di $l \geqq 0$ contiene elementi del tipo seguente:

(a) Per $z_{k+1}$ un polinomio $F\left(z_{k+1} /(z)_{k}\right)$ irriducibile in $\mathbb{D}_{k}\left[z_{k+1}\right]$ déll' anello $\mathbb{D} k\left[z_{k+1}^{p l}\right]$ che è privilegiato sopra $\mathbb{P}_{k}$.

(a) Per $i=2,3, \ldots, n-k$ un polinomio "monoidale» in $z_{k+1}$ della forma:

$$
D(z)_{k} z_{k+i}^{p^{i}}-G_{k+i}\left(z_{k+1} ;(z)_{k}\right)
$$

dove $D \in \mathbb{D}_{k z}$ e $G_{k+i} \in \mathbb{D}_{k}\left[z_{k+1}^{p^{l}}\right]$

9.6. Sui teoremi 25 e 26 poggia la cosidetta rappresentazione monoidale di una varietà analitica. A questo proposito dimostriamo il seguente teorema, cfr. [12], pag. 58 .

\section{Thorema 27.}

Sia $\mathbb{D}$ un corpo perfetto con infiniti elementi; e sia $\mathfrak{p}$ un ideale primo nell' anello delle serie di potenze $\mathbb{P}_{n}\left(\mathfrak{p} \neq \mathbb{P}_{n}\right)$. Con le notazioni del teorema 25 (risp. 26) sia $\mathfrak{q}$ l'ideale in $\mathbb{P}_{n}$ con la seguente base:

(a) nel caso di caratteristica 0 :

$$
F, D z_{k+i}-G_{k+i}, \quad i=2,3, \ldots, n-k,
$$

(b) nel caso di caratteristica $p$ :

$$
F, D z_{k+i}^{p^{l}}-G_{k+i}, \quad i=2,3, n-k .
$$

Una serie $f$ di $\mathbb{P}_{a}$ appartiene a $\mathfrak{p}$ allora e soltanto allora che per una potenza $D^{x}$ di $D$ vale:

(a) nel caso di caratteristica 0 :

$$
D^{x} \cdot f \in \mathfrak{q}
$$

(b) nel caso di caratteristica $p$ :

$$
D^{*} \cdot f^{p l} \in \mathfrak{q} .
$$


Basta considerare il caso $p>0$, perchè il caso $p=0$ si ottiene formalmente da quello, ponendo $l=0$.

(1) Poichè $\mathfrak{q} \subset \mathfrak{p}$, da $D^{\alpha} \cdot f^{p l} \in \mathfrak{q}$, segue anche $D^{\alpha} f^{p l} \in \mathfrak{p}$. E poichè peraltro $D$, come elemento di $\mathbb{p}_{k}$, non appartiene a $\mathfrak{p}$, e $\mathfrak{p}$ è primo, $f$ appartiene a $\mathfrak{p}$.

(2) Per dimostrare il viceversa, basta riferirsi agli elementi di una base di $\mathfrak{p}$; prendiamo quindi la base dei polinomi in $\overline{\mathbb{p}}_{k, \mathfrak{n}-k}$. Sia $f \in \bar{p}=\mathfrak{p} \cap$ $\cap \overline{\mathbb{P}}_{k, n-k}$. Allora $f^{p^{l}}$ appartiene a $\mathbb{P}_{k}\left[z_{k+1}^{p^{l}}, z_{k+2}^{p^{l}}, \ldots, z_{n}^{\left.p^{l}\right]}\right.$. Da $D z_{k+i}^{p^{l}} \equiv G_{k+i} \bmod \mathbf{q}$ segue ora che, per un opportuno $\alpha$, il polinomio $D^{x} \bar{f}^{p l} \bmod \mathfrak{q} \otimes$ congruo ad un polinomio $\Gamma$ dell'anello $\mathbb{D}_{k}\left[z_{k+1}^{p^{l}}\right]$. Siccome $\mathfrak{q}$ è contenuto in $\overline{\mathfrak{p}}, \Gamma$ appartiene a $\bar{p} \cap \mathbb{P}_{k}\left[z_{k+1}\right]$. Questo ideale è peraltro un ideale principale e viene generato da $F \in \mathfrak{q}$. Perciò $\Gamma$ e quindi anche $D^{x} \bar{f}^{p l}$ è un elemento di $\mathfrak{q}$.

9.7. Ci occuperemo infine delle relazioni tra ideali primi diversi, allo scopo di dimostrare l'ugnaglianza del numero $k$ con la dimensione di un ideale primo nell' anello $\mathbb{D}_{n}$, come si è affermato nel teorema 24. Questo numero $k$ viene calcolato per l'ideale primo $\mathfrak{p}$ come nella dimostrazione del teorema 20 dopo aver eseguito la $\mathbb{P}$-trasformazione di coordinate $L$, che, per il teorema 24, è anche una premessa per la costruzione della base di polínomi in $\overline{\mathbb{P}}_{k, n-k}$ (cfr. teor. 24). Chiamiamo $L$ «trasformazione di coordinate di $\mathfrak{p}$ ». Il numero $k$, calcolato mediante $L$, sia indicato con $k(\mathfrak{p}, L)$ per significare, che non è finora esclusa la sua dipendenza da $L$. Per la dimensione di $\mathfrak{p}$ adoperiamo la notazione $\rho(\mathfrak{p})$. La nostra affermazione si esprime allora:

$$
k(\mathfrak{p}, L)=\rho(\mathfrak{p}) .
$$

1. Risultato Partale. - Siano $\mathfrak{p}$ e $\mathfrak{q}$ ideali primi in $\mathbb{P}_{n}\left(\mathfrak{q} \neq \mathbb{P}_{n}\right)$. Sia $\mathfrak{p}$ una parte propria di $\mathfrak{q}$. Sia $L$ una trasformazione di coordinate di $p$. Allora esiste una trasformazione di coordinate $L^{\prime}$ di $\mathfrak{q}$, tale che :

$$
k\left(\mathfrak{q}, L^{\prime}\right)<k(\mathfrak{p}, L)
$$

Sia $f$ un elemento di $\mathfrak{q}$ non appartenente a $p$. Secondo la 9.2. sussiste una congruenza :

$$
f^{m}+c_{1} f^{m-1}+\ldots+c_{m-1} f+c_{m} \equiv 0 \bmod \mathfrak{p}
$$

$c_{i} \in \mathbb{P}_{k}(k=k(\mathfrak{p}, L))$ per $i=1,2, \ldots, m, c_{m} \neq 0$. Da $f \in \mathfrak{q}$ e $\mathfrak{p} \subset \mathfrak{q}$ segne $c_{m} \in \mathfrak{q}$. Dunque $\mathfrak{q} \cap \mathbb{P}_{k}$ \&े diversu dall'ideale nullo. Il metodo seguente per la dimostrazione del teorema 20 si puo applicare nei primi $n-k$ passi anche per $q$ Siccome tuttavia $\mathfrak{q} \cap \mathbb{D}_{k} \neq(0)$, per $q$ seguirà ancora almeno un $(n-k+1)$-mo passo. Da ciò segue (1). 
2. Risultato parzialee. - Per ogni trasformazione di coordinate $L$ di $\mathfrak{p}$ vale la relazione

$$
p(\mathfrak{p}) \leqq k(\mathfrak{p}, L)
$$

Posto $\rho=\rho(\mathfrak{p})$, sia $\mathfrak{p} \subset \mathfrak{p}_{1} \subset \ldots \subset \mathfrak{p}_{\rho}$ una catena crescente di ideali primi con $p+1$ terinini. Per la (1) vale, con opportune trasformazioni di coordinate $L_{1}, L_{2}, \ldots, L_{\beta}$ :

$$
0 \leqq k\left(\mathfrak{p}_{\rho}, L_{\rho}\right)<k\left(\mathfrak{p}_{\rho-1}, L_{\rho-1}\right)<\ldots<k\left(\mathfrak{p}_{1}, L_{1}\right)<k(\mathfrak{p}, L) .
$$

Una tale catena di $\rho+1$ disuguaglianze tra interi porta come conseguenza la (2).

Per dimostrare l'inversa della (2), costruiamo una catena crescente di ideali primi con $k(\mathfrak{p}, L)+1$ termini.

3. Risultato Parzialie. - Sia $L$ una trasformazione di ooordinate di $\mathfrak{p}$ e $k=k(\mathfrak{p}, L)$. Con le notazioni del teorema 24 sia $\mathfrak{a}=\left(\mathfrak{p}, y_{k}\right)$. Vale allora la relazione:

$$
\mathbf{a}^{*}=\mathfrak{a} \cap \mathbb{p}_{k-1} y=(0) .
$$

(a) Diamo una dimostrazione indiretta. Sia $h(y)_{k-1} \in \mathfrak{a}^{*}$. Allora $h$ possiede una lappresentazione:

$$
h^{\prime}=p+y_{k} g
$$

con $p \in \mathfrak{p}$ e $g \in \mathbb{P}_{n}$. Per il teorema 24 è $g \equiv \bar{g} \bmod \mathfrak{p}$ con $\bar{g} \in \overline{\mathbb{P}}_{k, n-k}$. Da ciò segue $h \equiv y_{k} \bar{g} \bmod \mathfrak{p}$ e perciò anche $\bmod \overline{\mathfrak{p}}$, poiche $h-y_{k} \bar{g} \in \overline{\mathbb{P}}_{k, n-k}$ e $\mathfrak{p} \cap \overline{\mathbb{p}}_{k, n-k}=\overline{\mathfrak{p}}$. Quando $h-y_{k} \bar{g}$ è indipendente da $y_{n}$, tralasciamo la seguente parte $(b)$ della dimostrazione e proseguiamo con $(c)$.

(b) Costruiamo il risultante $\bar{R}$ di $h-y_{k} \bar{g}$ e $\overline{p_{n}}$ rispetto ad $y_{n}$ (cir. teorema 24). Essendo $\bar{p}_{n}$ irriducibile, questo risultante può annullarsi soltanto quando $h-y_{k} \bar{g}$ è divisibile per $\bar{p}_{n}$. Una relazione $h-y_{k} \bar{g}=\bar{p}_{n} \bar{q}$ con $\underline{q} \in \overline{\mathbb{P}}_{k, n-k}$ porge pertanto una formula falsa quando si ponga $y_{k}=0$. Essendo $\bar{p}_{n}$ e $h-y_{k} \bar{g}$ elementi di $\bar{p}, \bar{R}$ appartiene a $\mathfrak{p}^{-} \cap \overline{\mathbb{p}}_{k, n-k-1}$ con $\overline{\mathbb{P}}_{k, n-k-1}=$ $=\mathbb{P}_{k}\left[y_{k+1}, y_{k+2}, \ldots, y_{n-1}\right]$. Nella rappresentazione di $\vec{R}$ mediante determinante di SyLvester si legge facilmente che $\bar{R}=h^{m}-y_{k} \overline{\mathfrak{p}}_{1}$, dove $m$ è il grado di $\bar{p}_{n}$ e $\overline{\mathfrak{p}}_{1} \in \overline{\mathbb{P}}_{k, n-k-1}$. Esiste quindi un elemento $h_{1}=h^{m}$ in $\mathfrak{a}^{*}$, che $\dot{\mathrm{e}} \neq 0$ ed è congruo mod $\overline{\mathbf{p}}$ ad un polinomio $y_{k} \overline{\mathfrak{p}}_{2}$ con $\overline{\mathfrak{p}}_{1} \in \overline{\mathbb{P}}_{k, n-k-1}$.

(c) Poichè ora $h_{1}-y_{k} \overline{\mathbf{p}}_{1}$ è indipendente da $y_{,}$, ripetiamo l'argomentazione $(b)$ sostituendo $\bar{p}_{n}$ con $\bar{p}_{n-1}$. Nello stesso modo eliminiamo $y_{n-2}, \ldots, y_{k+1}$. Nell'ultimo passo troviamo una serie $h_{n-k} \in \mathfrak{a}^{*}$, che $\dot{\theta} \neq 0$ ed è congrua $\bmod \overline{\mathfrak{p}}$ ad un elemento $y_{k} \overline{\mathfrak{p}}_{n-k}$ con $\overline{\mathfrak{p}}_{n-k} \in \mathbb{P}_{k}$. Ora $h_{n-k}(y)_{k-1}-y_{k} \overline{\mathfrak{p}}_{n-k}(y)_{k}$ dovrebbe 
stare $\operatorname{in}_{\alpha}^{\leftarrow} \mathbb{P}_{k} \cap \overline{\mathbf{p}} ;$ ma questa intersezione è $(0)$, e $h_{n-k}-y_{k} \overline{\mathbf{p}}_{n-k} \otimes$ certo $\neq 0$, poichè $h_{n-k}$ non dipende da $y_{k}$. Questa contradizione dimostra il risultato parziale 3.

4. Risultato parziale, - Tra le componenti primarie di a ce n'è almeno una, la cui intersezione con $\mathbb{D}_{k-1}$ è l'ideale nullo.

Sia $\mathfrak{a}=\mathfrak{q}_{1} \cap \mathfrak{q}_{2} \cap \ldots \cap \mathfrak{q}_{\mathbf{r}}$ una rappresentazione di a mediante componenti primarie. Se ora fosse, per $i=1,2, \ldots, r, q_{i} \neq 0$ un elemento di $\mathfrak{q}_{i} \cap \boldsymbol{p}_{k-1}$, sarebbe $q_{1} \cdot q_{2} \ldots q_{r} \in \mathfrak{a} \cap \mathbb{D}_{k-1}$ in contradizione con il risultato parziale 3 .

Ђ. Risultato Panziale. - Vale la relazione:

$$
k(\mathbf{p}, L) \leqq \mathfrak{p}
$$

Sia $\mathfrak{q}_{1}$ una componente primaria di a con $\mathfrak{q}_{1} \cap \mathbb{p}_{k-1}=(0)$. Essendo $\mathfrak{p}_{1}$ l'ideale primo di $\mathfrak{q}_{1}$, vale anche $\mathfrak{p}_{1} \cap \mathbb{p}_{k-1}=(0)$, perchè per una potenza $\mathfrak{p}_{1}{ }^{\sigma}$ di $\mathfrak{p}_{1}$ vale $\mathfrak{p}_{1}{ }^{\circ} \subset \mathfrak{q}_{1}$. D'altra parte $\mathfrak{p}_{1}$ contiene l'ideale $a$ quindi anche $\mathfrak{p}$. Per questo $\mathrm{i}$ polinomi $\bar{p}_{k+i}, i=1,2, \ldots, n-k$, stanno in $\mathrm{p}_{1}$, e inoltre $y_{k} \in \mathfrak{p}_{1}$. Essendo $\mathfrak{p}_{1} \cap \mathbb{P}_{k-2}=(0)$ segue ora :

$$
k\left(\mathfrak{p}_{1}, L\right)=k(\mathfrak{p}, L)-1
$$

Applicando la stessa argomentazione a $\mathfrak{p}_{1}$ ecc., si trova una catena crescente di ideali primi :

$$
\mathfrak{p} \subset \mathfrak{p}_{1} \subset \mathfrak{p}_{2} \subset \ldots \subset \mathfrak{p}_{k(\mathfrak{w}, L)},
$$

tale che $k\left(\mathfrak{p}_{i}, L\right)=k(\mathfrak{p}, L)-i$. Questa catena ha $k(\mathfrak{p}, L)+1$ termini. Da ciò segue (4).

Da (2) e (4) si ottiene l'affermazione $9(8)$.

9.8. Il risultato $9(8)$, dimostrato dapprima soltanto per gli ideali primi, vale per gli ideali arbitrari.

Teorema 28.

Sia $\mathbb{P}$ un corpo con infiniti elementi, e sia a un ideale nell'anello delle serie di potenze $\mathbb{P}_{n}\left(a \neq \mathbb{P}_{\text {..) }}\right.$. Dopo una $\mathbb{P}$-trasformazione di coordinate a possegga una base di polinomi nell'anello $\overline{\mathbb{P}}_{k, n-k}$ tale che a $\cap \mathbb{D}_{k}=(0)$. Allora $k$ è pari alla dimensione di a, cfr. 4.3.

La base di polinomi esiste in forza dei teoremi 20 e 14. Essendo $\mathfrak{a} \cap \mathbb{D}_{k}=(0)$, segne come nel risultato parziale 4 l'esistenza di almeno una componente di $\mathbf{a}$, arente dimensione $\geqq k$. $\dot{\mathrm{E}}$ dunque $\rho \geqq k$. Se ora fosse $\rho>k$, esisterebbe una componente primaria $q$ di $a$ con $q \cap \boldsymbol{p}_{k+1}=(0)$. E per essere a $\subset \mathfrak{q}$ vale la relazione $a \cap \mathbb{p}_{k+1}=(0)$. Ma questa formala è falsa : i dunque $\rho=k$. 
9.9. Dai risultati parziali 1 e 5 segue il teorema:

Teorema 29.

(a) Sia $\mathfrak{p}$ un ideale primo nell'anello delle serie di potenze $\mathbb{P}_{n}$ della dimensione $p \geq 1$. Allora esiste un ideale primo $\mathfrak{p}_{1}$ in $\mathbb{P}_{n}$, che contiene $\mathfrak{p}$ ed ha la dimensione $p-1$. (b) Siano $\mathfrak{p}$ e $\mathfrak{p}_{1}$ ideali grimi in $\mathbb{D}_{n}\left(\mathfrak{p}_{1} \neq \mathbb{D}_{n}\right)$. Da $\mathfrak{p} \subset \mathfrak{p}_{1}$ $e . p \neq \mathfrak{p}_{1}$, segue che la dimensione di $\mathfrak{p}_{1}$ è minore della dimensione di $\mathfrak{p}$.

Questi teoremi verranino approfonditi nel seguito.

\section{\& 10. - Varietà Algebroldi.}

10.1. Dopo avere illustrato la struttura di un ideale primo nell'anello delle serie di potenze, deve essere esaminato il medeșimo problema perideali generali. Come nel paragrafo precedente prendiamo come anello dei coefficienti $1 \mathrm{P}$ un corpo. 11 problema si enuncia: Sia dato un ideale a nell' anello delle serie di potenze $\mathbb{P}_{n}$. Si calcolino gli ideali primari $\mathfrak{q}_{t}$ in na rappresentazione $: \mathfrak{a}=\mathfrak{q}_{1} \cap \mathfrak{q}_{2} \cap \ldots \cap \mathfrak{q}_{r}$. Eccezion fatta per alcuni semplici casi speciali, poco si può dire in generale sulla soluzione di questo problema. Cerchiamo quindi di delimitare il problema. Pensando alle necessità della geometria algebrica, $\otimes$ immediato richiedere soltanto la determinazione delle componenti isolate di $\mathfrak{a}$. La componente $\mathfrak{q}_{i}$ si dice isolata se non esiste alcuna $\mathfrak{q}_{j}$ con $j \neq i$, in modo che per gli ideali primi $\mathfrak{p}_{i}$ e $\mathfrak{p}_{j}$ di $\mathfrak{q}_{i}$, risp. $\mathfrak{q}_{j}$, valga la relazione: $\mathfrak{p}_{j} \subset \mathfrak{p}_{i}$. Una componente non isolata si dice immersa.

Il calcolo delle componenti isolate viene ricondotto al corrispondente problema algebrico per gli ideali di polinomi. Per la sua soluzione l'algebra applica il metodo dell' eliminazione; metodo che non fornisce in generale le componenti isolate primarie, ma soltanto i loro ideali primi. Perciò è opportuno circoscrivere ancor più il nostro compito della decomposizione e richiedere semplicemente il calcolo di quegli ideali primi $\mathfrak{p}_{i}$ che appartengono alle componenti isolate $\mathfrak{q}_{i}$ di $a$. A questa delimitazione del nostro problema si addice una particolare terminologia, precisamente la terminologia delle « varieta algebroidi ». L'impiego delle varietà algebroidi porta, in ultima analisi, che sono da esaminare soltanto gli ideali primi di componenti isolate di un ideale di serie di potenze. Definiremo dapprima questi concetti.e, nel prossimo paragrato, analizzeremo il problema della decomposizione.

10.2. Sia a un ideale dell' anello delle serie di potenze $\mathbb{P}_{n}$. Se $\mathfrak{a} \neq \mathbb{P}_{n}$, associamo ad $\mathfrak{a}$ un insieme $d \dot{i}$ "punti», che chiamiamo "varietà algebroide di a » indichiamo con $M(\mathfrak{a})$. I «punti » di $M(\mathfrak{a})$ saranno ideali primi di $\mathbb{P}_{n}$. L'ideale primo $\mathfrak{p}$ di $\phi_{n}$ definirk un punto di $M(\mathfrak{a})$ allora e soltanto allora che a appartiene a $\mathfrak{p}$. La dimensione di $M(\mathfrak{a})$ sarà uguale alla dimensione di a. 
Il punto $\mathfrak{p}$ di $M(\mathfrak{a})$ si dirà *punto generale» di $M(\mathfrak{a})$ se $M(\mathfrak{a})=M(\mathfrak{p})$. Ogni ideale primo possiede un punto generale.

10.3. Valgono $i$ seguenti teoremi :

(a) $D a$ a $\subset \mathfrak{b}$ segue $M(\mathfrak{b}) \subset M(\mathfrak{a})$.

La dimostrazione segue subito delle definizioni.

(b) $S e \mathfrak{a}=\mathfrak{b} \cap \mathfrak{c}$, risulta $M(\mathfrak{a})=M(\mathfrak{b}) \cup M(\mathfrak{c})$.

Da $\mathfrak{a} \subset \mathfrak{p}$ segue $\mathfrak{b} \cdot \mathfrak{c} \subset \mathfrak{p}$. Poichè $\mathfrak{p}$ è un ideale primo, si ha $\mathfrak{b} \subset \mathfrak{p}$ oppure $\mathfrak{c} \subset \mathfrak{p}$, e quindi è $\mathfrak{p} \in M(\mathfrak{b}) \cup M(\mathfrak{c})$. Se è viceversa $\mathfrak{p} \in M(\mathfrak{b}) \cup M(\mathfrak{c})$, risulta $\mathfrak{p} \in M(\mathfrak{b})$ o $\mathfrak{p} \in M(\mathcal{C})$. Nel primo caso è $\mathfrak{b} \subset \mathfrak{p}$ e quindi anche $\mathfrak{a} \subset \mathfrak{p} ;$ nel secondo caso è $\mathfrak{C} \in \mathfrak{p}$ e cosi pure $\mathfrak{a} \in \mathfrak{p}$.

(c) Se $\mathfrak{a}=(\mathfrak{b}, \mathfrak{c})$, risulta $M(\mathfrak{a})=M(\mathfrak{b}) \cap M(\mathfrak{c})$.

Da $\mathfrak{a} \subset \mathfrak{p}$ segue $\mathfrak{b} \subset \mathfrak{p}$ e $\mathfrak{C} \subset \mathfrak{p}$, e quindi $\mathfrak{p} \in M(\mathfrak{b}) \cap M(\mathcal{c})$. Se viceversa 8 $\mathfrak{p} \in M(\mathfrak{b}) \cap M(\mathcal{c})$, risulta $\mathfrak{p} \in M(\mathfrak{b})$ e $\mathfrak{p} \in M(\mathfrak{c})$, e quindi $\mathfrak{b} \subset \mathfrak{p}$ e $\mathfrak{c} \subset \mathfrak{p}$. Vale percio anche $\mathfrak{a} \subset \mathfrak{p}$.

(d) Se $\mathfrak{q}$ è un ideale primario con l'ideale primo $\mathfrak{p}$ risulta $M(\mathfrak{q})=M(\mathfrak{p})$.

Essendo $\mathfrak{q} \subset \mathfrak{p}$, si ha subito $M(\mathfrak{p}) \subset M(\mathfrak{q})$. D'altro canto da $\mathfrak{q} \subset \mathfrak{p}_{1}$ segue anche $\mathfrak{p} \subset \mathfrak{p}_{1}$; percio è $M(\mathfrak{q}) \subset M(\mathfrak{p})$. Anche un ideale primario possiede quindi un punto generale.

(e) Se l'ideale a possiede le componenti primarie isolate $\mathfrak{q}_{i}$ con $i$ corri. spondenti ideali primi $\mathfrak{p}_{i}, i=1,2, \ldots, r$, vale la relazione:

$$
M(\mathfrak{a})=M\left(\mathfrak{p}_{1}\right) \cup M\left(\mathfrak{p}_{2}\right) \cup \ldots \cup M\left(\mathfrak{p}_{r}\right)
$$

La formula segue da (a), (b) e (d).

(f) Se l'ideale a possiede la base $f_{1}, f_{2}, \ldots, f_{s}$, vale la:

$$
M(\mathfrak{a})=M\left(f_{1}\right) \cap M\left(f_{2}\right) \cap \ldots \cap M\left(f_{s}\right) .
$$

Segue da (c).

10.4. Una varieta algebroide $M(\mathfrak{a})$ si dice riducibile, quando $M(\mathfrak{a})$ əे l'unione di due varietà algebroidi, nessuna delle quali contiene l'altra. Se non esiste alouna decomposizione di $M(\mathfrak{a})$ di questo tipo, la varietà $M(\mathfrak{a})$ si dice irriducibile.

Teorema 30.

Sia $\mathbb{D}$ un corpo. Per gli ideali a dell' anello delle serie di potenze $\mathbb{D}_{n}$ 
$\mathfrak{a} \neq \mathbb{P}_{n}$ sussiste la proposizione: La varietà algebroide $M(\mathfrak{a})$ è irriducibile allora e soltanto allora che essa possegga un punto generale, cfr. 10.2.

(a) Sia $M(\mathfrak{a})=M(\mathfrak{b}) \cup M(\mathfrak{c})=M(\mathfrak{p})$, dove $\mathfrak{a}, \mathfrak{b}, \mathfrak{c}$ sono ideali e $\mathfrak{p}$ è un ideale primo. Per 10.3. (b) si ha $M(\mathfrak{b} \cap \mathfrak{c})=M(\mathfrak{p})$, onde $\mathfrak{b} \cap \mathfrak{c} \subset \mathfrak{p}$ e quindi $\mathfrak{b} \cdot \mathfrak{c} \subset \mathfrak{p}$. Poichè $\mathfrak{p}$ è un ideale primo, o $\mathfrak{b}$ o $c$ appartiene a $\mathfrak{p}$. Supponiamo che sia $\mathfrak{b} \subset \mathfrak{p}$; risulta allora $M(\mathfrak{p}) \subset M(\mathfrak{b})$. Inoltre è $M(\mathcal{C}) \subset M(\mathfrak{p})$, da cui segue $M(\mathfrak{c}) \subset M(\mathfrak{b})$. Se $M(\mathfrak{a})=M(\mathfrak{p})$, ogni decomposizione ha cosi come conseguenza che una delle varietà parziali fa parte dell'altra.

(b) Se nella $10(1)$ è $r \geq 2$, poniamo $\mathfrak{b}=\mathfrak{p}_{1}$ e $c=\mathfrak{p}_{2} \cap \mathfrak{p}_{3} \cap \ldots \cap \mathfrak{p}_{r}$. 亡̀ allora $M(\mathfrak{a})=M(\mathfrak{b}) \cup M(\mathfrak{c})$. Da $M(\mathfrak{b}) \subset M(\mathfrak{c})$ seguirebbe $\mathcal{C} \subset \mathfrak{p}_{1}$; e per almeno un $i \geqq 2$ dovrebbe essere $\mathfrak{p}_{i} \subset \mathfrak{p}_{1}$. Ma ciò è escluso, trattandosi di componenti isolate. Viceversa, da $M(\mathfrak{c}) \subset M(\mathfrak{b})$ si otterrebbe $M\left(\mathfrak{p}_{i}\right) \subset M\left(\mathfrak{p}_{1}\right)$ per $i \geqq 2$, e quindi $\mathfrak{p}_{1} \subset \mathfrak{p}_{i} ;$ ma ciò è impossibile per il medesimo motivo. Nel caso $r \geq 2$ la varietà $M(\mathfrak{a})$ è dunque riducibile.

Nel caso $r=1 M(a)$ è irriducibile, come è stato dimostrato in (a). In pari tempo abbiamo trovato il seguente teorema:

TEorema 31.

Sia ID un corpo. La varietà algebroide di un qualunque ideale nell' anello delle serie di potenze $\mathbb{\Phi}_{n}$ è rappresentabile in modo unico come unione di varietà algebroidi irriducibili.

10.5. La serie $f$ dell' anello delle serie di potenze $\mathbb{D}_{n}$ si dirà nulla nel punto $\mathfrak{p}$, se $f$ appartiene a $\mathfrak{p}$. La serie $f$ si dirà nulla sulla varietà algebroide $M(\mathfrak{a})$, se $f$ gi annulla in tutti $\mathbf{i}$ punti di $M(\mathfrak{a})$.

Teorema 32. (Teorema degli zeri di HilberT).

Nell'anello delle serie di potenze $\mathbb{P}_{n}$ sia a un ideale $\neq \mathbb{P}_{n}$ ed $M(\mathfrak{a})$ la sua varietà algebroide. Se la serie $f d i \mathbb{P}_{n}$ si annulla su $M(\mathfrak{a})$, esiste una potenza $f^{\sigma}$ di $f$ cppartenente ad a.

Se $\mathfrak{a}=\mathfrak{q}_{1} \cap \mathfrak{q}_{2} \cap \ldots \cap \mathfrak{q}_{r}$ è una rappresentazione di a mediante ideali primari, e $\mathfrak{p}_{i}$ l'ideale primo di $\mathfrak{q}_{i}(i=1,2, \ldots, r)$, la $f$ si annulla in particolare nei punti $\mathfrak{p}_{i}$, cioè $f \in \mathfrak{p}_{i}$ per $i=1,2, \ldots, r$. Per le proprietà degli ideali primari esiste una potenza $f \sigma_{i}$ appartenente a $\mathfrak{q}_{i}$; se $\sigma$ è il massimo dei $\sigma_{1}$, $\sigma_{2}, \ldots, \sigma$, vale la relazione $f^{\sigma} \in \mathfrak{q}_{i}$ per $i=1,2, \ldots, r$, e quindi $f^{\sigma} \in \mathfrak{a}$.

\section{§ 11. - Decomposizione degli ideali di serie di potenze.}

11.1. Sotto determinate ipotesi peI l'anello di serie di potenze a il calcolo delle componenti irriducibili della varietà algebroide $M(\mathfrak{a})$ si può conseguire con mezzi algebrici. 
Teorema 33.

(a) Notazioni ed ipotesi.

Sia $\mathbb{P}$ un corpo, e $\mathbb{P}_{k+i}$ l'anello delle serie di potenze $\mathbb{P}\left[\left[z_{1}, z_{2}, \ldots, z_{k}\right.\right.$, $\left.w_{1}, w_{2}, \ldots, w_{i}\right], i \geq 1, \mathbb{P}_{k}=\mathbb{P}_{k} z ;$ sia inoltre $\overline{\mathbb{P}}_{k, l}$ l'anello dei polinomi $\mathbb{P}_{k}\left[w_{1}, w_{2}\right.$, $\left.\ldots, w_{l}\right]$. In $\mathbb{D}_{k+l}$ sia dato un ideale $\mathfrak{a}\left(\neq \mathbb{D}_{k+l}\right)$ con la seguente proprietà : per $i=1,2, \ldots, l$, a contenga un polinomio $p_{i} \in \mathbb{P}_{k+i-1}\left[w_{i}\right]$, che sia privilegiato sopra $\mathbb{P}_{k+i-1}$.

Per il teorema 14 a possiede una base $\left(\bar{a}_{1}, \bar{a}_{2}, \ldots, \bar{a}_{r}\right)$ nell'anello $\overline{\mathbb{D}}_{k, l}$. Sia $\overline{\mathfrak{a}}$ l'ideale con la base $\left(\bar{a}_{1}, \bar{a}_{2}, \ldots, \bar{a}_{r}\right)$ in $\overline{\mathbb{P}}_{k, l}$. Poichè la base di $\overline{\mathfrak{a}}$ è anche base per $\mathfrak{a}, \mathrm{l}$ 'ideale esteso di $\overline{\mathfrak{a}}$ in $\mathbb{P}_{k+l}$ coinoide con $\mathfrak{a}: \mathfrak{a}=\mathbb{P}_{k+l} \cdot \overline{\mathfrak{a}}$.

Sia $\bar{w}$ l'ideale $\left(w_{1}, w_{2}, \ldots, w_{l}\right)$ in $\overline{\mathbb{P}}_{k, l}$. Per il teorema 22 l'ideale $\overline{\mathfrak{a}}$ pos. siede una decomposizione in ideali primari :

$$
\overline{\mathfrak{a}}=\overline{\mathfrak{q}}_{\mathbf{1}} \cap \overline{\mathfrak{q}}_{2} \cap \ldots \cap \overline{\mathfrak{q}}_{8} \cap \overline{\mathbf{c}}
$$

con le seguenti proprietà : per $i=1,2, \ldots, s$ vale la relazione $\left(\overline{\mathfrak{q}}_{i}, \overline{\mathbf{W}}\right) \neq \overline{\mathbb{P}}_{k, l}$, $\Theta$ inoltre $(\overline{\mathfrak{c}}, \overline{\mathfrak{W}})=\overline{\mathbb{D}}_{k, l}$. Sia infine $\mathfrak{q}_{i}$ (per $i=1,2, \ldots, s$ ) l'ideale esteso $\mathbb{P}_{k+l} \cdot \overline{\mathfrak{q}}_{i}$ di $\overline{\mathfrak{q}}_{i}$ in $\mathbb{D}_{k+l}$.

(b) Tesi.

(1) Gli ideali $\mathfrak{q}_{i}, i=1,2, \ldots, s$, sono primari; inoltre vale la relazioue:

$$
\mathfrak{q}_{i} \cap \overline{\mathbb{P}}_{k, l}=\overline{\mathfrak{q}}_{i}, \quad i=1,2, \ldots, s .
$$

(2) L'ideale a possiede una decomposizione in ideali primari:

$$
\mathfrak{a}=\mathfrak{q}_{1} \cap \mathfrak{q}_{2} \cap \ldots \cap \mathfrak{q}_{8} \cap \mathfrak{q}^{\prime} \cap \mathfrak{q}^{\prime \prime} \cap \ldots \cap \mathfrak{q}^{(t)} .
$$

Ciascuna delle componenti aggiuntive $\mathfrak{q}^{\prime}, \mathfrak{q}^{\prime \prime}, \ldots, \mathfrak{q}^{(t)} \dot{e}$ immersa in una delle componenti $\mathfrak{q}_{1}, \mathfrak{q}_{2}, \ldots, \mathfrak{q}_{s}$, ciò̀ se l'ideale primo $\mathfrak{p}^{(j)}$ appartiene all'ideale primario $\mathfrak{q}^{(j)}$, esiste un ideale primario $\mathfrak{q}_{i}$ con l'ideale primo $\mathfrak{p}_{i}$, tale che $\mathfrak{p}_{i} \subset \mathfrak{p}^{(j)}$.

(a) Il corollario al teorema 14 mostra che a contiene un polinomio $\bar{p}_{i}$ $(i=1,2, \ldots, l)$ dell'anello $\mathbb{D}_{k}\left[w^{1}, w_{2}, \ldots, w_{i}\right]$, il quale, come polinomio in $w_{i} \grave{e}$ privilegiato sopra $\mathbb{D}_{k+i-1} . D a \bar{a} \subset \overline{\mathfrak{q}}_{h}$ segue mediante estensione in $\mathbb{P}_{k+l}: \mathfrak{a} \subset \mathfrak{q}_{h}$; l'ideale $\mathfrak{q}_{h}$ contiene percio anche $i$ polinomi $\bar{p}_{i}$. Il teorema 21 , applicato per $\bar{R}_{l}=\overline{\mathbb{D}}_{k, l}$ e $R_{l}=\mathbb{\mathbb { D }}_{k+l}$ porge allora la $11(2)$; e quindi questi polinomi appar. tengono anche a $\overline{\mathfrak{q}}_{h}$. Dal teorema 23 segue ora che $\mathfrak{q}_{h}$ è un ideale primario dell' anello $\mathbb{P}_{k+l}$. Oon cio resta dimostrata la prima affermazione.

(b) Dalle 11(1), 8.2. (a) e 1.3. (c) segue:

$$
\mathbf{a}=\mathbb{p}_{k+l} \cdot \overline{\mathbf{a}} \subset \mathfrak{q}_{1} \cap \mathfrak{q}_{2} \cap \ldots \cap \mathfrak{q}_{\mathbf{l}} .
$$


Consideriamo gli ideali primari aggiuntivi $\mathfrak{q}^{(j)}, j=1,2, \ldots, t$, dell'ideale $11(3)$. Poichè $\mathfrak{q}^{(j)}$ contiene l'ideale a e quindi i polinomi $\bar{p}_{i}$, $\mathfrak{q}^{(j)}$ possiede, per il teorema 14, una base in $\overline{\mathbb{P}}_{k, l}$; base che appartiene anche a $\overline{\mathfrak{q}}^{(j)}=\overline{\mathbb{P}}_{k, l} \cap \mathbf{q}^{(j)}$, per cui vale la relazione $\mathfrak{q}^{(j)}=\mathbb{P}_{k+l} \cdot \overline{\mathfrak{q}}^{(j)}$. Per quanto visto in 1.3. $\left\langle b_{j}, \overline{\mathfrak{q}}^{(j)}\right.$ è un ideale primario in $\overline{\mathbb{P}}_{k, l}$. Per $1.3(a)$ segue dalle $11(3)$ e 11(2):

$$
\overline{\mathbb{P}}_{k, l} \cap \mathfrak{a}=\overline{\mathfrak{q}}_{\mathbf{2}} \cap \overline{\mathfrak{q}}_{2} \cap \ldots \overline{\mathfrak{q}}_{s} \cap \overline{\mathfrak{q}}^{\prime} \cap \overline{\mathfrak{q}}^{\prime \prime} \cap \ldots \cap \overline{\mathfrak{q}}^{(t)} .
$$

Facendo l'intersezione con $\overline{\mathfrak{c}}$, segue dalla $11(1)$ e da $\overline{\mathfrak{a}} \subset \overline{\mathbb{D}}_{k, l} \cap$ a la relazione:

$$
\overline{\mathfrak{a}}=\overline{\mathfrak{q}}_{1} \cap \overline{\mathfrak{q}}_{2} \cap \ldots \cap \overline{\mathfrak{q}}_{s} \cap \overline{\mathfrak{q}}^{\prime} \cap \overline{\mathfrak{q}}^{\prime \prime} \ldots \cap \overline{\mathfrak{q}}^{(t)} \cap \overline{\mathbf{c}} .
$$

Confrontiamo ora le formule $11(1)$ e (3), la seguente considerazione essendo valida per $j=1,2, \ldots, t$. Dalle $11(1)$ e (3) segue :

$$
\overline{\mathfrak{q}}_{1} \cap \overline{\mathfrak{q}}_{2} \cap \ldots \cap \overline{\mathfrak{q}}_{s} \cap \overline{\mathfrak{c}} \subset \overline{\mathfrak{q}}^{(j)} \text {. }
$$

Per la proprietà fondamentale degli ideali primari, $\overline{\mathfrak{q}}^{(j)}$ contiene allora una potenza di uno degli ideali $\overline{\mathfrak{q}}_{1}, \overline{\mathfrak{q}}_{2}, \ldots, \overline{\mathfrak{q}}_{s}$ o $\overline{\mathbf{c}}$. Escludiamo dapprima la possibilità $\widetilde{C}^{p} \subset \overline{\mathbf{q}}^{(j)}$ per ogni $\rho$. Accanto a $\bar{W}$ definiamo gli ideali $\mathfrak{3}=\left(z_{1}, z_{2}, \ldots, z_{k}\right)$ in $\mathbb{P}_{k+l}, \mathfrak{W}=\left(w_{1}, w_{2}, \ldots, w_{l}\right)$ in $\mathbb{P}_{k+l}$, e $\overline{\mathfrak{z}}=\left(z_{1}, z_{2}, \ldots, z_{k}\right)$ in $\overline{\mathbb{P}}_{k, l}$. Allora $\mathrm{e}$ $\mathfrak{q}^{\prime j)} \subset(\mathbf{3}, \mathfrak{W})$ perchè $(\xi, \mathfrak{W})$ è l'ideale massimo di $\mathbb{D}_{k+l}$ (efr. 3.5.), e di qui segue $\overline{\mathfrak{q}}^{(j)} \subset(\overline{\mathbf{3}}, \overline{\mathbf{w}})$. Se ora per un certo $\rho$ vale la relazione $\overline{\mathfrak{c}}^{\circ} \subset \overline{\mathfrak{q}}^{(j)}$, se ne deduce:

$$
\left.(\overline{\mathbf{C}} p, \overline{\mathbf{w}}) \subset\left(\overline{\mathbf{q}^{(i)}}, \overline{\mathbf{w}}\right) \subset \overline{(3}, \overline{\mathbf{w}}\right) .
$$

Essendo $(\overline{\mathcal{C}}, \overline{\mathbb{W}})=\overline{\mathbb{D}}_{k, l}$ esistono elementi $\bar{c} \in \overline{\mathrm{C}}$ e $\overline{\boldsymbol{\omega}} \in \overline{\mathrm{W}}$, tali che $1=\bar{c}-\bar{\omega}$, e allora $\dot{\theta}(1+\bar{\omega}) \rho \in \overline{\mathcal{C}} \rho$ e quindi $1 \in(\overline{\bar{C}}, \bar{W})$. Dovrebbe così essere $1 \in(\overline{3}, \bar{w})$, ciò che è assurdo. Rimane quindi soltanto la possibilità che $\overline{\mathbf{q}}^{(j)}$ contenga una potenza di un ideale primario $\overrightarrow{\mathfrak{q}}_{i}$. Dalla terza formula in 1.3. (c) segue $\mathfrak{q}_{i} \subset \mathfrak{q}^{(j)}$, e di qui la relazione per il relativo ideale primo: $\mathfrak{p}_{i} \subset \mathfrak{p}^{(j)}$, che appunto si voleva dimostrare.

11.2. Nelle ipotesi del teorema 33 si ottengono gli ideali primi delle componenti isolate dell' ideale delle serie di potenze a mediante estensione degli ideali primi delle componenti isolate dell'ideale di polinomi $\overline{\mathfrak{a}}$. $\dot{\mathrm{E}}$ interessante ed importante che in questo calcolo si può essere partiti da una base arbitraria di a in $\overline{\mathbb{D}}_{k, l}$. Un cambiamento in questa base comporta soltanto dei cambiamenti nelle componenti immerse di $\bar{a}$ oppure nell'ideale $\bar{c}$. La scelta più favorevole di questa base sarebbe evidentemente quella per cui $\overline{\mathbf{a}}=\overline{\mathbf{p}}_{k, l} \cap \mathfrak{a}$; in tal caso manca nella $11(1)$ l'ideale $\overline{\mathbf{c}}$, come segue immediatamente dalla 11(3). Nei singoli casi concreti non si potrà tuttavia trovare facilmente una base di $\overline{\mathbf{p}}_{k, l} \cap \mathfrak{a}$. 
Usando le notazioni del $\S 10$ possiamo stabilire:

Nelle ipotesi del teorema 33, la determinazione della varietà algebroide $M(\mathfrak{a})$ è ricondotta alla determinazione della varietà algebrica $\bar{M}(\overline{\mathfrak{a}})$ appartenente all'ideale di polinomi $\overline{\mathfrak{a}}$. La decomposizione di $\bar{M}(\overline{\mathfrak{a}})$ nelle sue componenti irriducibili si realizza con il metodo dell' eliminazione. Non pud qui essere nostro compito il presentare le diverse possibilità che si presentano; perciò si rimanda in particolare al chiaro metodo di VAN DER WAERDEN in [20], pag. 116, i risultati più importanti per noi sono:

(1) Dal calcolo scaturiscono anzitutto le componenti irriducibili $\bar{M}(\overline{\mathbf{p}})$, per cui è $\overline{\mathfrak{p}} \cap \mathbb{D}_{k}=(0)$. Le dimensioni (algebriche) di queste componenti (sul corpo quoziente di $\mathbb{P}_{k}$ ) sono perciò $\geq 0$.

(2) Il calcolo porge inoltre un sistema di elementi $b_{1}, b_{2}, \ldots, b_{\beta}$ di $\mathbb{D}_{k}$ con la seguente proprietà : Sia $\mathfrak{b}$ l'ideale $\left(\mathfrak{a}, b_{1}, b_{2}, \ldots, b_{\beta}\right)$. Poichè $\mathfrak{b} \cap \mathbb{D}_{k} \neq(0)$, la varieta algebroide $M(\mathfrak{b})$ contiene soltanto componenti irriducibili $M(\mathfrak{p})$ con $\mathfrak{p} \cap \mathbb{p}_{k} \neq 0$. Tra esse sono tutte le componenti irriducibili isolate di $M(\mathfrak{a})$ con questa proprietà. Oltre quelle $M(\mathfrak{b})$ può avere anche altre componenti irriducibili; le quali appartengono allora anche a $M(\mathfrak{a})$, essendo $M(\mathfrak{b}) \subset M(\mathfrak{a})$ in quanto $\mathfrak{a} \subset \mathfrak{b}$.

11.3. Il metodo algebrico per il calcolo di $M(\mathfrak{a})$ è applicabile soltanto per ideali che contengono i polinomi dati nel teorema 33. Nel più importante caso particolare, quello in cui $\mathbb{P} \grave{e}$ un corpo con infiniti elementi, si possono soddisfare queste ipotesi per $\mathfrak{a}$ secondo il teorema 20 dopo una $\mathbb{P}$-trasformazione di coordinate e allora anche per $\mathfrak{b}$ dopo l'eliminazione (cfr. 11.2). In questo caso il metodo algebrico di eliminazione rende quindi possibile la determinazione di tutte le componenti irriducibili della varietà algebroide.

\section{§ 12. - La teoria della funzione norma.}

12.1. La teoria della funzione norma rende possibile un intimo approfondimento nella conoscenza delle varietà algebroidi.

\section{Definizione della funzione norma.}

$R$ sia un dominio d'integrità noetheriano con modulo; ed $\bar{R}_{t}$ sia l'anello di polinomi $R\left[w_{1}, w_{2}, \ldots, w_{l}\right]$. Sia ora $\overline{\mathfrak{p}}$ un ideale primo in $\bar{R}_{l}$ con le seguenti proprietà :

$$
\overline{\mathfrak{p}} \cap R=(0)
$$

(b) Per $j=1,2, \ldots, l, \overrightarrow{\mathfrak{p}}$ contenga un polinomio $\bar{p}_{j}$ nelle $w_{1}, w_{2}, \ldots, w_{j}$, il quale, come polinomio in $w_{j}$ abbia il coefficiente di grado massimo uguale ad 1. 
Indichiamo con $\omega_{j}$ la classe resto di $w_{j} \bmod \bar{p}$. Fssendo $u_{1}, u_{2}, \ldots, u_{l}$ indeterminate, poniamo:

$$
z=u_{1} w_{1}+u_{2} w_{2}+\ldots+u_{l} w_{l}
$$

$\Theta$

$$
\zeta=u_{1} \omega_{1}+u_{2} \omega_{2}+\ldots+u_{l} \omega_{l} .
$$

$\zeta$ è intero algebrico sopra l'anello $R\left[u_{1}, u_{2}, \ldots, u_{l}\right]$ e radice di un polinomio irriducibile:

$$
\Phi=\Phi(\overline{\mathbf{p}})=\Phi\left(z, u_{1}, u_{2}, \ldots, u_{l}\right)
$$

dell' anello $R\left[u_{1}, u_{2}, \ldots, u_{l}, z\right]$, efr. [4], pag. 131, [19], e [20], pag. 122. Come polinomio in $z, \Phi$ ha il coefficiente del termine di grado massimo nguale ad 1. Chiameremo questo polinomio $\Phi(\overline{\mathfrak{p}})$ funzione norma dell'ideale $\overline{\mathfrak{p}}$.

12.2. Unitamente parliamo ora di un metodo di calcolo di $\Phi$, ofr. [19].

\section{Relazioni tra risultante e funzione norma.}

Sia

$$
\bar{f}_{1}, \bar{f}_{2}, \ldots, \bar{f}_{r}
$$

una base di $\bar{p}$. Agginngiamo alla base i polinomi $\bar{p}_{j}, j=1,2, \ldots, l$, nel caso che essi non siano già presenti in essa.

Si calcolerà il sistema risultante di KRONECKER (cfr. [4], pag. 45) rispetto a $w_{l}$ di $\bar{f}_{1}, \overline{f_{2}}, \ldots, \bar{f}_{r}, \bar{p}_{l}$ e:

$$
z-u_{1} w_{1}-u_{2} w_{2}-\ldots-u_{l} w_{l} .
$$

Avendo $\bar{p}_{l}$, come polinomio in $w_{l}$ il coefficiente del termine di grado mas. simo uguale ad 1, si può eseguire questa eliminazione, cfr. [4], nota a piè di pag. 45. Il risultato $\dot{A}$ un sistema di polinomi dell'anello $R\left[u_{1}, u_{2}, \ldots\right.$, $\left.u_{l}\right]\left[w_{1}, w_{2}, \ldots, w_{l-1}\right]$ :

$$
\bar{g}_{1}, \bar{g}_{2}, \ldots, \bar{g}_{s}
$$

Da questi polinomi e da $\bar{p}_{l-1}$ eliminiamo con lo stesso metodo $w_{l-1}$ ecc. Dopo l'eliminazione di $w_{l}, w_{l-1}, \ldots, w_{1}$, si ottiene un sistema di polinomi

$$
\bar{h}_{1}, \bar{h}_{2}, \ldots, \bar{h}_{t}
$$


dell'anello $R\left[u_{1}, u_{2}, \ldots, u_{l}, z\right]$. Poichè il sistema $\left(\zeta, \omega_{1}, \omega_{2}, \ldots, \omega_{l}\right) \grave{e}$ uno zero di $12(3)$ e $12(4)$, esso annulla 12(5) ece. e alla fine anche 12(6), e percio tntti i polinomi $\bar{h}_{i}$ sono divisibili per $\Phi(\overline{\mathfrak{p}})$ :

$$
\bar{h}_{i}=(\Phi(\overline{\mathfrak{p}}))^{v_{i}} \cdot \bar{\varphi}_{i}, \quad v_{i} \geq 1, i=1,2, \ldots, t .
$$

essendo $\Phi(\overline{\boldsymbol{p}})$ e $\bar{\varphi}_{i}$ senza fattori comuni.

A questa dedazione si può obbiettare che uno dei sistemi risultanti $\bar{g}_{i}, \ldots, \bar{h}_{j}$ si potrebbe annullare identicamente. Ma dalla proprietà di questo sistema risultante seguirebbe, che $\overrightarrow{\mathfrak{p}}$ avrebbe un zero di grado di trascendenza $\geqq 1$ in un ampliamento algebrico del corpo quoziente $K$ di $R$. Tuttavia la dimensione algebrica di $\overline{\mathfrak{p}}$ sopra $K$ è 0 , perchè $\overline{\mathfrak{p}}$ contiene $\mathrm{i}$ polinomi $\bar{p}_{j}$ $(j=1,2, \ldots, t)$. Poichè $\overline{\mathbf{p}}$ è primo, i polinomi $\bar{\varphi}_{i}, i=1,2, \ldots, t$, posseggono al massimo divisori comuni appartenenti all' anello $K\left[u_{1}, u_{2}, \ldots, u_{l}\right]$.

12.3. Alla funzione norma $\Phi(\overline{\mathfrak{p}})$ è collegato il seguente ideale dell'anello $\bar{R}$ :

\section{L'ideale di annullamento della funzione norma.}

Introduciamo in $\Phi(\overline{\mathfrak{p}})$ per $z$ l'espressione $12(1)$ e ordiniamo secondo i prodotti $U_{y}$ di $u_{1}, u_{2}, \ldots, u_{l}$. Si ha allora:

$$
\begin{gathered}
\Phi\left(u_{1} w_{1}+u_{2} w_{2}+\ldots+u_{l} w_{l}, u_{1}, u_{2}, \ldots, u_{l}\right)= \\
=\sum_{y=1}^{N} \bar{a}_{y}\left(w_{1}, w_{2}, \ldots, w_{l}\right) U_{y} .
\end{gathered}
$$

I coefficienti $\bar{a}_{\nu}$ sono polinomi dell'anello $\bar{R}_{l}$. L'ideale a di $\bar{R}_{l}$ con la base $\bar{a}_{1}, \bar{a}_{2}, \ldots, \bar{a}_{N}$ si chiamerà «l'ideale di annullamento della funzione norma $\Phi(\overline{\mathfrak{p}})$ ».

12.4. Dimostriamo alcune proprietà di $\bar{a}$.

(a) $\grave{E} \overline{\mathfrak{a}} \subset \overline{\mathfrak{p}}$.

Poichè $\zeta$ è uno zero di $\Phi$, il sistema $\left(\omega_{1}, \omega_{2}, \ldots, \omega_{\ell}\right)$ è uno zero di $\bar{a}$. È quindi $\overline{\mathbf{a}} \equiv 0 \bmod \overline{\mathfrak{p}}$ e ciò significa ohe $\overline{\mathbf{a}} \subset \overline{\mathfrak{p}}$.

(b) $\overline{\mathbf{a}}$ contiene per $j=1,2, \ldots, l$, un polinomio $\bar{q}_{j} d i w_{j}$ con coefficienti in $R$ e coefficiente del termine di grado massimo uguale ad 1.

In un ampliamento algebrico opportunamente scelto $\Phi$ si decompone nel prodotto di fattori lineari che sono coniugati sopra il corpo quoziente di $\mathrm{R}\left[u_{1}, u_{2}, \ldots, u_{l}\right]$ :

$$
\Phi=\prod_{\nu=1}^{m}\left(z-u_{1} \omega_{1}^{(\nu)}-u_{2} \omega_{2}^{(\nu)}-\ldots-u_{l} w_{l}^{(\nu)}\right)
$$


Dimostriamo l' affermazione seguente:

Tutte le quantità $\omega_{j}^{(\nu)}, v=1.2, \ldots, m, j=1,2, \ldots, l$ sono indipendenti dalle $u_{1}, u_{2}, \ldots, u_{l}$.

Per $j=1$ le $\omega_{1}{ }^{(\nu)}, \nu=1,2, \ldots, m$, sono zeri del polinomio $\bar{p}_{1}\left(w_{1}\right)$ e perciò indipendenti dalle $u_{1}, u_{2}, \ldots, u_{l}$. Supponiamo già dimostrata l'indipendenza delle $\omega_{1}^{(v)}, \omega_{2}^{(v)}, \ldots, \omega_{j-1}^{(v)}(j \geq 2)$ dalle $u_{1}, u_{2}, \ldots, u_{l}$. La medesima indiṕendenza delle $\omega_{j}^{(v)}$ segue dal fatto, che $\omega_{j}^{(v)}$ è uno zero del polinomio $\bar{p}_{j}\left(w_{j}, \omega_{1}{ }^{(v)}, \omega_{2}{ }^{(v)}\right.$, $\ldots, \omega_{j-2}^{(v)}$ ). Pur $i=l$ si ottiene la nostra affermazione, cfr. [20], pag. 120.

Nel caso inseparabile non è necessario che i fattori lineari di $\Phi$ in 12(9) siano tutti distinti. Sostituendo qui $z$ con $12(1)$, si riconosce che tra i polinomi $\bar{a}_{i}$ vi sono anche i polinomi:

$$
\bar{q}_{j}=\prod_{\nu=1}^{m}\left(w_{j}-\omega_{j}^{(\nu)}\right), \quad j=1,2, \ldots, l,
$$

e questi hanno la proprietà richiesta. Del resto $\bar{q}_{j}$ è una potenza del polino. mio irriducibile con la radice $\omega_{j}$ perchè le $\omega_{j}^{(v)}$ sono coniugati sopra il corpo quoziente di $R$.

(c) Se $\overline{\mathfrak{q}}$ è un ideale primo in $\bar{R}_{l}$, da $\overline{\mathbf{a}} \subset \overline{\mathfrak{q}}$ segue $\overline{\mathfrak{p}} \subset \overline{\mathfrak{q}}$.

$\overline{\mathfrak{a}}$ possiede quindi una sola componente isolata, e il suo ideale primo è $\overline{\mathfrak{p}}$. Questa proprietà si può anche formulare al modo seguente :

Esiste un intero $m \geq 1$, tale che

$$
\overline{\mathbf{p}}^{m} \subset \overline{\mathbf{a}} .
$$

Se infatti $\overline{\mathfrak{a}}=\bar{g}_{1} \cap \bar{g}_{2} \cap \ldots \cap \bar{g}_{\text {, }}$ è la decomposizione di $\overline{\mathbf{a}}$ in ideali primari e $\overline{\mathfrak{p}}_{i}$ ə l'ideale primo di $\bar{g}_{i}$, da (c) segue $\overline{\mathfrak{p}} \subset \overline{\mathfrak{p}}_{i}$ per $i=1,2, \ldots$, r. Per le proprietà degli ideali primari una potenza $\overline{\mathfrak{p}}^{m_{i}}$ appartiene a $\bar{g}_{i}$; e se $m$ è il massimo dei numeri $m_{1}, m_{2}, \ldots, m_{r}$; vale la $12(11)$.

L'affermazione (c) sarà dimostrata più tardi, cfr. 12.8. Si osservi da ultimo che, naturalmente, la funzione norma $\Phi(\overline{\mathfrak{p}})$ rimane univocamente determinata da $\bar{p}$; ebbene il risultato (c) moistra ohe anche viceversa la funzione norma individua anivocamente l'ideale primo $\bar{p}$.

12.5. L'utilità della funzione norma si manifesta in teoremi di specializzazione.

Sia $\mathfrak{i}$ un ideale primo nell'anello $R$; $\Theta$ sia $\overline{\mathfrak{i}}$ l'ideale esteso di $\mathfrak{i}$ nell'anello di polinomi $\bar{R}_{l}=R\left[w_{1}, w_{2}, \ldots, w_{l}\right]$. Indichiamo con $R^{*}$ l'anello delle classi resto $R / \mathfrak{i}$. Il passaggio alle classi resto mod $\mathfrak{i}$ genera un omomorfismo di $R$ su $R^{*}$, che si estende all'anello dei polinomi sopra $R$ sostituendo ai coefficienti dei polinomi le loro classi resto mod î. Così l'anello $\bar{R}_{l}^{*}=R^{*}\left[w_{1}, w_{2}, \ldots, w_{l}\right]$ 
proviene da $\bar{R}_{l}$. Nelle indagini seguenti il risultato di questo omomorfismo verrà contrassegnato con un asterisco.

12.6. Premettiamo alcuni lemmi, per cui si veda [7], pag. 27.

(a) Se $\overline{\mathfrak{q}} \grave{e}$ un ideale primo di $\bar{R}_{l}$ con $\mathfrak{i} \subset \overline{\mathfrak{q}}$, allora $\mathfrak{q}^{*} \dot{e}$ un ideale primo $d i \bar{R}_{l}^{*}$. Se inoltre si suppone $\overline{\mathfrak{q}} \cap R=\mathfrak{i}$, risulta $\mathfrak{q}^{*} \cap R^{*}=(0)$.

Da $f^{*} g^{*} \in \mathfrak{q}^{*}$ segue, per due qualunque rappresentanti $\bar{f}, \bar{g}$ delle classi resto $f^{*}$ risp. $g^{*}$, la relazione $\bar{f} \cdot \bar{g} \in(\overline{\mathfrak{q}}, \overline{\mathfrak{i}})=\overline{\mathfrak{q}}$. Siccome $\overline{\mathfrak{q}} \dot{e}$ un ideale primo, si ha $\bar{f} \in \overline{\mathfrak{q}}$ oppure $\bar{g} \in \overline{\mathfrak{q}}$; nel primo caso risulta $f^{*} \in \mathfrak{q}^{*}$, nel secondo $g^{*} \in \mathfrak{q}^{*}$. $\mathfrak{q}^{*}$ è dunque primo. Nell' ipotesi $\mathfrak{q} \cap R=\mathfrak{i}$ sia $t^{*} \in \mathfrak{q}^{*} \cap R^{*}$. Esistono allora degli elementi $\bar{f} \in \overline{\mathfrak{q}}, \bar{b} \in \overline{\mathfrak{f}}$ e $a \in R$, in modo che $\bar{f}=a+\bar{b}$ è un rappresentante di $f^{*}$. È dunque $a \in(\overline{\mathfrak{q}}, \overline{\mathfrak{i}}) \cap R=\overline{\mathfrak{q}} \cap R=\mathfrak{i}$, da cui segue $f^{*}=0$.

(b) In $\bar{R}_{l}^{*}$ sia $\mathfrak{q}^{*}$ l'ideale primo con la base $q_{1}^{*}, q_{2}^{*}, \ldots, q_{\alpha^{*}}^{*}$; e sia $\mathfrak{q}^{*} \cap R^{*}=(0)$. Per $i=1,2, \ldots, \alpha$ scegliamo un rappresentante $\overline{q_{i}}$ di $q_{i}^{*}$ in $\overline{R_{l}}$. L'ideale $\mathfrak{q}^{-}=\left(\overline{q_{1}}, \overline{q_{2}}, \ldots, \overline{q_{x}}, \overline{\mathfrak{i}}\right) \grave{e}$ allora primo in $\overline{R_{l}}$ e vale la relazione $\overline{\mathfrak{q}} \cap R=\mathfrak{t}$.

Da $\bar{f} \bar{g} \in \overline{\mathfrak{q}}$ segue $f^{*} g^{*} \in \mathfrak{q}^{*}$. Essendo $\mathfrak{q}^{*}$ un ideale primo, risulta $f^{*} \in \mathfrak{q}^{*}$ oppure $g^{*} \in \mathfrak{q}^{*}$. Nel primo caso si ha $\bar{f} \in(\overline{\mathfrak{q}}, \overline{\mathfrak{i}})=\overline{\mathfrak{q}}$ e nel secondo $\bar{g} \in(\overline{\mathfrak{q}}, \overline{\mathfrak{i}})=\overline{\mathfrak{q}}$; $\overrightarrow{\mathbf{q}}$ risulta perciò un ideale primo. Se $\bar{h} \in \overline{\mathfrak{q}} \cap R$, si ha $h^{*} \in \mathfrak{q}^{*} \cap R^{*}=(0)$. Tatti gli elementi di $R$ che mediante l'omomorfismo vengono rappresentati nello 0 , appartengono ad $\mathfrak{i}$ perciò $\bar{h} \in \mathfrak{i}$; e quindi $\overline{\mathfrak{q}} \cap R=\mathfrak{i}$.

(c) Siano $p(x)$ e $q(x)$ polinomi dell'anello $\bar{R}_{1}=R[x]$ con coefficiente del termine di grado massimo uguale ad $1 ;$ e sia $H$ il loro risultante. Allora $H^{*}$ $\grave{e}$ il risullante di $p^{*}(x)$ e $q^{*}(x)$.

Questo teorema segue, per es., dalla rappresentazione del risultante mediante il determinante di Sylvester. Il teorema vale anche per più di due polinomi relativamente al sistema risultante di KRONECKER.

12.7. Segue il fondamentale teorema sulle funzioni norma, cfr. [19].

Teorema 34.

Sia $R$ un dominio d'integrità noetheriano con modulo. Nell' anello di polinomi $\bar{R}_{l}=R\left[w_{1}, w_{2}, \ldots, w_{l}\right]$ sia $\overrightarrow{\mathfrak{p}}$ un ideale primo con le proprietà (a) $e(b)$ di 12.1. e con la funzione norma $\Phi(\overline{\mathfrak{p}})$. Sia $\mathfrak{i}$ un ideale primo di $R$ ed $\bar{R}_{l} \rightarrow \bar{R}_{l}^{*}$ l'omomorfismo descritto in 12.5. L'ideale primo $\overrightarrow{\mathfrak{p}}$ viene allora rap. presentato sull'ideale primo $\mathfrak{p}^{*}$ di $\bar{R}_{l}^{*} ;$ e la funzione norma $\Phi(\overline{\mathfrak{p}})$ va nel polinomio $\Phi^{*}(\overline{\mathfrak{p}}, \mathfrak{i})$.

(a) Sia $\overline{\mathfrak{q}}$ un ideale primo di $\bar{R}_{l}$ tale che $\overline{\mathfrak{p}} \subset \overline{\mathfrak{q}}$ e $\overline{\mathfrak{q}} \cap R=\mathfrak{i}$. Se $\mathfrak{q}^{*} \dot{e}$ l'immagine di $\mathfrak{q}$, risulta $\mathfrak{p}^{*} \subset \mathfrak{q}^{*}$ e $\mathfrak{q}^{*} \cap R^{*}=(0)$.

L'ideale primo $\mathfrak{q}^{*}$ possiede una funzione norma $\Phi^{*}\left(\mathfrak{q}^{*}\right)$, e questa $\grave{e}$ un divisore irriducibile $d i \bar{i} \Phi^{*}(\overline{\mathfrak{p}}, \mathfrak{i})$. 
(b) Se $\Phi_{1}{ }^{*}$ è un divisore irriducibile di $\Phi^{*}(\overline{\mathbf{p}}, \mathfrak{i})$, esiste un ideale primo $\overline{\mathfrak{q}}$ di $\bar{R}_{l}$ per cui $\overline{\mathfrak{p}} \subset \overline{\mathfrak{q}}$ e $\overline{\mathfrak{q}} \cap R=\mathfrak{i}$, tale che $\Phi_{1}{ }^{*} \dot{e}$ la funzione norma dell' im. magine $\mathfrak{q}^{*}$ di $\overline{\mathfrak{q}}$.

(a) Le affermazioni contenute in (a) seguono da 12.6. (a), eccettnata l'nltima affermazione. Per dimostrare quest' ultima consideriamo l'ideale $\mathfrak{a}^{*}$, che è l'immagine dell'ideale di annullamento $\overline{\mathfrak{a}}$ di $\Phi(\overline{\mathfrak{p}})$, cfr. 12.3. Sia $\omega_{i}^{*}$ la classe resto di $w_{j} \bmod \mathfrak{q}^{*}$ nell'anello delle classi resto $\bar{R}_{l}^{*} \mathfrak{q}^{*}$ (per $\left.j=1,2, \ldots, l\right)$. Dato che $\Phi^{*}\left(\mathfrak{q}^{*}\right)$ è il polinomio irriducibile con lo zero $\zeta^{*}=u_{1} \omega_{1}^{*}+u_{i} \omega_{2}^{*}+\ldots$ $+u_{l} \omega_{l}^{*}$, è sufficiente dimostrare che $\Phi^{*}(\overline{\mathfrak{p}}, \cdot \mathfrak{i})$ si annulla per $\zeta^{*}$. Ora ciò si ottiene facilmente dal collegamento di $\Phi^{*}(\overline{\mathfrak{p}}$. $\mathfrak{i})$ con $a^{*}$ e dalla relazione $\mathfrak{a}^{*} \subset \mathfrak{p}^{*} \subset \mathfrak{q}^{*}$ che segue da 12.4. (a).

(b) Per dimostrare le affermazioni contenute in (b) applichiamo il lemma 12.6. (c) alla proprietà risultante della funzione norma (cfr. 12.3.) e l'omomorfismo $\bar{R}_{l} \rightarrow \bar{h}_{l}^{*}$. Si tratta anzitutto dell'eliminazione di $w_{l}$ da $12(4)$ e $f_{1}^{*}, f_{2}^{*}, \ldots, f_{r}^{*}, p_{l}^{*}$, cfr. $12(3)$; il risultato $\dot{e} g_{1}^{*}, g_{2}{ }^{*}, \ldots, g_{s}^{*}$, cfr. 12(5). Da questi polinomi e da $p_{l-1}^{*}$ si eliminerà $w_{l-1}$ ecc. Dopo aver eliminato $w_{l}, w_{l-1}$, $\ldots, w_{1}$ si ottiene alla fine $h_{1}^{*}, h_{2}^{*}, \ldots, h_{t}^{*}$, cfr. $12(6)$; e questi polinomi hanno il divisore $\Phi^{*}(\overline{\mathfrak{p}}, \mathfrak{i})$ e percio anche $\Phi_{1}{ }^{*}$. Sia ora $\zeta^{*}$ uno zero di $\Phi_{1}^{*}$ in un ampliamento algebrico opportunamento scelto del corpo quoziente di $R^{*}\left[u_{1}, u_{2}, \ldots, u_{l}\right]$. Per la proprietà risultante del sistema $h_{1}^{*}, h_{2}^{*}, \ldots, h_{t}^{*}$ e dei precedenti sistemi risultanti possiamo calcolare a partire da $\zeta^{*}$, le quantita $\partial_{1}^{*}, \partial_{2}^{*}, \ldots, \partial_{l}^{*}$, che annullano questi sistemi. Come in 12.4. (b) si mostra, che le quantità $\partial_{j}^{*}$ sono indipendenti dalle $u_{i}$ perchè sono zeri dei polinomi $p_{j}^{*}$ che non contengono alcuna delle $u_{i}$. Da 12(4) e 12(3) segue allora :

(1): $\zeta=u_{1} \partial_{1}{ }^{*}+u_{2} \partial_{2}{ }^{*}+\ldots+u_{l} \partial_{l}^{*}$ e (2): Il sistema $\partial^{*}=\left(\partial_{1}{ }^{*}, \partial_{2}{ }^{*}, \ldots, \partial_{l}{ }^{*}\right)$ è uno zero di $f_{1}^{*}, f_{2}^{*}, \ldots, f_{r}^{*}$, e quindi di $p^{*}$. Sia ora $q^{*}$ la totalita di tutti $i$ polinomi di $\bar{R}_{l}^{*}$ che si annullano per $\partial^{*}$; naturalmente $\mathfrak{q}^{*}$ è un ideale primo ed è $\mathfrak{q}^{*} \cap R^{*}=(0)$. Come è stato ora dimostrato, è anche $\mathfrak{p}^{*} \subset \mathfrak{q}^{*}$. Poichè allora $\mathrm{q}^{*}$ contiene il polinomio $p_{j}^{*}$, per $j=1,2, \ldots, l$, e poichè $\mathfrak{q}^{*} \cap R^{*}=(0)$, è definita la funzione norma di $\mathfrak{q}^{*}$. Essa è il polinomio irriducibile con lo zero $\zeta^{*}$ e coincide quindi con $\Phi_{1}{ }^{*}$. Definito ora $\overline{\mathfrak{q}}$ esattamente come in 12.6. (b), segue che $\overline{\mathfrak{q}}$ è un ideale primo di $\bar{R}_{l}$, ed è $\overline{\mathfrak{q}} \cap R=\mathbf{i}$. Da $\mathfrak{p}^{*} \subset \mathfrak{q}^{*}$ segue inoltre $\overline{\mathbf{p}} \subset \overline{\mathbf{q}}$. Con ciò è dimostrata anche l'ultima parte del teorema 34 .

12.8. Unitamente si può dimostrare l'affermazione 12.4. (c) sull'ideale di annullamento $\overline{\mathfrak{a}}$ della funzione norma $\Phi(\overline{\mathfrak{p}})$. Sia $\overline{\mathfrak{q}}$ un ideale primo di $\bar{R}_{l}$ per cai $\overline{\mathbf{a}} \subset \overline{\mathfrak{q}}$; posto $\mathfrak{i}=\overline{\mathfrak{q}} \cap R$, consideriamo, come in 12.5., l'omomorfismo $\overleftarrow{R}_{l} \rightarrow \breve{R}_{l}^{*}$. Per 12.6. (a) $\mathfrak{q}^{*}$ è un ideale primo, ed è $\mathfrak{q}^{*} \cap R^{*}=(0)$; di qui e da 12.4. (b) segue che è definita la funzione norma $\Phi^{*}\left(\mathfrak{q}^{*}\right)$. Se $\omega_{j}^{*}$ (per $j=1$, $2, \ldots, l)$ è la classe resto di $w_{j} \bmod \mathfrak{q}^{*}$ nell' anello delle elassi resto $R_{l}^{*} / \mathfrak{q}^{*}$, allora $\Phi^{*}\left(\mathbf{q}^{*}\right)$ ¿ il polinomio irriducibile con lo zero $\zeta^{*}=u_{1} \omega_{1}{ }^{*}+u_{2} \omega_{2}{ }^{*}+\ldots$ 
$\ldots+u_{l} \omega_{l}^{*}, \mathrm{E}$ siccome il sistema $\left(\omega_{1}^{*}, \omega_{2}^{*}, \ldots, \omega_{l}^{*}\right)$ è uno zero di $\mathfrak{q}^{*}$ ed $\mathfrak{a}^{*}$ appartiene a $\mathfrak{q}^{*}$, a eansa di $\mathfrak{a} \subset \mathfrak{q}, \zeta^{*}$ è anche zero di $\Phi^{*}(\overline{\mathfrak{p}}, \mathfrak{i})$, cfr. 12.7.; perciô $\Phi^{*}(\overrightarrow{\mathfrak{p}}, \mathfrak{i})$ è divisibile per $\Phi^{*}\left(\mathfrak{q}^{*}\right)$. L'affermazione $\overrightarrow{\mathfrak{p}} \subset \overline{\mathfrak{q}}$ segue ora dal teorema 34 (b) ove sia $\Phi_{1}^{*}=\Phi^{*}\left(\mathfrak{q}^{*}\right)$.

12.9. Trasportiamo queste definizioni e teoremi al caso, che a noi interessa, degli anelli di serie di potenze.

TEOREMA 35.

(1) Sia. $\mathbb{P}$ un corpo con infiniti elementi, e p un ideale primo nell'anello delle serie di potenze $\mathbb{P}_{n} \mathfrak{p} \neq \mathbb{P}_{n}$. Dopo la $\mathbb{P}$-trasformazione di coordinate $(y)_{n}=L(x)_{n}, \mathfrak{p}$ possegga una base di polinomi nell'anello di polinomi $\overline{\mathbb{p}}_{k, n-k}$, cfr. teorema 24. Essendo $\overline{\mathfrak{p}}=\mathfrak{p} \cap \overline{\mathbb{p}} k, n-k$, esiste allora la funzione norma $\Phi(\overline{\mathfrak{p}})$ dell' ideale primo $\bar{p}$. Posto:

$$
z=u_{k+1} y_{k+1}+u_{k+2} y_{k+2}+\ldots+u_{n} y_{n},
$$

$\Phi(\bar{p})$ risulta un polinomio dell'anello $\mathbb{P}_{k}\left[u_{k+1}, u_{k+2}, \ldots, u_{n}, z\right]$.

(2) $\overrightarrow{\mathfrak{a}}$ sia l'ideale di annullamento della funzione norma $\Phi(\vec{p})$ (cfr. 12.3.), ed a il suo estensione in $\mathbb{P}_{n}$. Risulta allora $\mathfrak{a} \subset \mathfrak{p} ;$ e le varietà algebroidi $M(\mathfrak{a})$ di a ed $M(\mathfrak{p})$ di $\mathfrak{p}$ coincidono. Per una potenza $\mathfrak{p}^{m}(m \geq 1)$ vale la relazione $\mathfrak{p}^{m} \subset$ a.

(3) Sia $\mathfrak{i}$ un ideale primo dell'anello di serie di potenze $\mathbb{D}_{k}, \mathfrak{i} \neq \mathbb{D}_{k}$, e $\mathbb{D}^{*}$ il dominio d'integrità delle classi resto $\mathbb{D}_{k} / \mathbf{i}$. Mediante l'omomorfismo $\mathbb{D}_{k} \rightarrow \mathbb{D}^{*}$, da $\overline{\mathbb{P}}_{k, n-k}$ nasce l'anello $\mathbb{P}_{n-k}^{*}=\mathbb{p}^{*}\left[y_{k+1}, y_{k+2}, \ldots, y_{n}\right]$. Inoltre $\overline{\mathfrak{p}}$ sia rappresentato su $\mathfrak{p}^{*}$ e $\Phi(\overline{\mathfrak{p}}) s u \Phi^{*}(\overline{\mathfrak{p}}, \mathfrak{i})$.

(3a) Sia $\mathfrak{q}$ un ideale primo di $\mathbb{p}_{n}$, tale che $\mathfrak{p} \subset \mathfrak{q}$ e $\mathfrak{q} \cap \mathbb{p}_{k}=\mathfrak{i}$. Allora $\overline{\mathbf{q}}=\mathfrak{q} \cap \overline{\mathbb{D}}_{k, n-k}$ è ideale primo di $\overline{\mathbb{D}}_{k, n-k}$, e l'ideale esteso di $\overline{\mathbf{q}}$ in $\mathbb{\mathbb { P }}_{n}$ coincide con $\mathfrak{q}$. Se $\mathfrak{q}$ viene rappresentato sopra $\mathfrak{q}^{*}$, risulta $\mathfrak{q}^{*} \cap \mathbb{p}^{*}=(0)$ e $\mathfrak{p}^{*} \subset \mathfrak{q}^{*} . L a$ funzione norma $\Phi^{*}\left(\mathfrak{q}^{*}\right)$ di $\mathfrak{q}^{*} \grave{e}$ allora un divisore irriducibile di $\Phi^{*}(\overline{\mathbf{p}}, \mathfrak{i})$.

(3b) Sia $\Phi_{1}{ }^{*}$ un divisore irriducibile di $\Phi *(\overline{\mathfrak{p}}, \mathfrak{i})$. Esiste allora un ideale primo $\mathfrak{q}$ di $\mathbb{P}_{n}$ per cui $\mathfrak{p} \subset \mathfrak{q}$ e $\mathfrak{q} \cap \mathbb{P}_{k}=\mathfrak{i}$, tale che $\Phi_{1}^{*}$ è la funzione norma dell'immagine $\mathfrak{q}^{*}$ di $\overline{\mathfrak{q}}=\mathfrak{q} \cap \overline{\mathbb{P}}_{k, n-k}$

(1) La primd affermazione segue dal teorema 24 e da 12.1.

(2) Sarà dimostrato che $M(\mathfrak{a})=M(\mathfrak{p})$, una volta che siano stabilite le due seguenti affermazioni: (2a) $a \subset \mathfrak{p}$, e (2b): Se $\mathfrak{q}$ è un ideale primo di $\mathbb{p}_{n}$ ed è $\mathfrak{a} \subset \mathfrak{q}$, è anche $\mathfrak{p} \subset \mathfrak{q}$. Da esse si ottiene, come in 12.4., anche l'ultima formula in (2). L'affermazione (2a) segue da 12.4(a). Per dimostrare la (2b) osserviamo: Per la 1.3. (b) $\overline{\mathfrak{q}}=\mathfrak{q} \cap \overline{\mathbb{D}}_{k, n-k}$ è ideale primo di $\overline{\mathbb{D}}_{k, n-k}$. Da $\mathfrak{a} \subset \mathfrak{q}$ segue $\overline{\mathfrak{a}} \subset \overline{\mathfrak{q}}$; e perció, per la 12.4 (c) risulta $\mathfrak{p} \subset \overline{\mathfrak{q}}$. L'ideale primo $\overline{\mathfrak{q}}$ contiene perciò i polinomi $\bar{p}_{k+j}, j=1,2, \ldots, n-k$, efr. teorema 24 . Per il 
teorema 14 l'ideale primo $\mathfrak{q}$ possiede una base in $\overline{\mathbf{p}}_{k, n-k}$, e quindi l'estensione di $\overline{\mathfrak{q}}$ in $\mathbb{P}_{n}$ coincide con $\mathfrak{q}$. L'affermazione $(2 b)$ segue ora per estensione da $\overrightarrow{\mathfrak{p}} \subset \overline{\mathbf{q}}$.

$3(\mathfrak{a}) \overline{\mathfrak{q}}$ è ideale primo per la 1.3. (b). La dimostrazione che $\mathfrak{q}=\mathbb{P}_{n} \overline{\mathfrak{q}}$, è contenuta nella dimostrazione di (2b). Le altre affermazioni esposte in (3a) conseguono del teorema $34(a)$. Nel caso (3b) il medesimo teorema 34 fornisce un ideale primo $\overline{\mathfrak{q}}$ in $\overline{\mathbb{p}}_{k, n-k}$ per cui $\overline{\mathfrak{p}} \subset \overline{\mathfrak{q}}$ e $\overline{\mathfrak{q}} \cap \mathbb{p}_{k}=\mathfrak{i}$. Sia allora $\mathfrak{q}$ l'ideale esteso di $\overline{\mathfrak{q}}$ in $\mathbb{P}_{,}$. Per dimostrare l'uguaglianza $\mathfrak{q} \cap \overline{\mathbb{P}}_{k, n-k}=\overline{\mathfrak{q}}$ applichiamo il teorema 23 (insieme con il corollario 1). L'ipotesi (a) di questo teorema è soddisfatta, in quanto, per essere $\overline{\mathfrak{p}} \subset \bar{q}$, $\mathfrak{q}$ contiene i polinomi $\bar{p}_{k+j}, j=1,2$, $\ldots, n-k$. Rimane da verificare l'ipotesi (b) del teorema 23.

Dimostriamo anzitutto che : se $m$ è l'ideale massimo $\left(y_{1}, y_{2}, \ldots, y_{n}\right)$ di $\mathbb{D}_{n}$, $\mathrm{m}^{*}=\left(y_{1}{ }^{*}, y_{2}{ }^{*}, \ldots, y_{n}{ }^{*}\right)$ è un ideale primo di $\mathbb{P}_{n-k}^{*}$. Infatti l'ideale $\overline{\mathfrak{m}}=\left(y_{1}, y_{2}\right.$, $\left.\ldots, y_{n}\right)$ di $\overline{\mathbb{D}}_{k, n-k} \dot{\text { è }}$ un ideale primo ed essendo $\mathfrak{i} \neq \mathbb{P}_{k}$, si ha anche $\mathfrak{i} \subset \overline{\mathfrak{m}}$. Da 12.6. (a) segue allora quanto asserito per $\mathrm{m}^{*}$. Proveremo ora che è soddisfatta l'ipotesi (b) del teorema 23: $L^{\prime}$ ideale $\left(\overline{\mathfrak{q}}, y_{k+1}, y_{k+2}, \ldots, y_{n}\right)$ e diverso da $\overline{\mathbb{D}}_{k, n-k}$. Da a $\subset \mathfrak{p}$ segue che a appartiene all'ideale massimo $\left(y_{1}, y_{2}, \ldots, y_{n}\right) \mathrm{di}$ $D_{n}$. Anche $\overline{\mathfrak{a}}$ appartiene all'ideale di $\overline{\mathbb{D}}_{k, n-k}$ avente questa base; l'immagine $a^{*}$ appartiene percio all'ideale primo $\mathrm{m}^{*}=\left(y_{1}{ }^{*}, y_{2}{ }^{*}, \ldots, y_{n}{ }^{*}\right)$ di $\mathbb{D}_{n-k}^{*}$. Sostituendo quindi in $\Phi^{*}(\overline{\mathfrak{p}}, \mathfrak{i})$ ai coefficienti le classi resto $\bmod \mathfrak{m}^{*}$, si ottiene una potenza $z^{m}$. Perche $\mathfrak{m}^{*}$ è un ideale primo $\mathbb{R}_{n-k}^{*} / \mathfrak{m}^{*}$ è un dominio d'integrita. Quindi anche $\Phi_{1}{ }^{*}$ deve arere la medesima proprietà di $\Phi^{*}(\overline{\mathfrak{p}}$, $\mathfrak{i})$, vale a dire $i$ coefficienti di $\Phi_{1}{ }^{*}$ - eccettuato il coefficiente del termine di grado massimo che è $1-$ sonó $\equiv 0 \bmod \mathfrak{m}^{*}$. Indicando con $\mathfrak{b}^{*} l^{\prime}$ ideale di annullamento di $\Phi^{*}{ }_{1}$ si ha $b^{*} \subset m^{*}$. Possiamo ora applicare a $b^{*}$ e $m^{*}$ il risnltato 12.4. (c), ottenendo $\mathfrak{q}^{*} \subset \mathfrak{m}^{*}$. Da ciò segue $\overline{\mathfrak{q}} \subset \overline{\mathrm{m}}$ e quindi $\left(\overline{\mathfrak{q}}, y_{k+1}, y_{k+2}, \ldots\right.$, $\left.y_{n}\right) \subset \overline{\mathfrak{m}}$. Dunque eे $\left(\bar{q}, y_{k+1}, y_{k+2}, \ldots, y_{n}\right) \neq \overline{\mathbb{p}}_{k, n-k}$.

Dal teorema 23 segue $\mathfrak{q} \cap \overline{\mathbb{P}}_{k, n-k}=\overline{\mathfrak{q}}$ e di qui $\mathfrak{q} \cap \mathbb{P}_{k}=\mathfrak{i}$, inoltre $\mathfrak{q}$ è un ideale primo.

Il teorema 35 resta con ciò dimostrato.

\section{§ 13. - Alcuni teoremi di intersezione.}

13.1. Come prima applicazione della teoria della funzione norma, dimostriamo alcuni teoremi di intersezione.

Terorema 36 .

Sia un corpo con infiniti elementi. Supponiamo che l'ideale primo $\mathfrak{p}$ nell'anello di serie di potenze $\mathbb{P}_{n}$ possegga una base di polinomi nell'anello di polinomi $\overline{\mathbb{D}}_{k, n-k}$, cfr. il teorema 24 . Se $\mathfrak{i}\left(\neq \mathbb{D}_{k}\right)$ è un ideale primo nell'anello 
delle serie di potenze $\mathbb{D}_{k}$ e $j$ il suo ideale esteso in $\mathbb{P}_{n}$, allora la varietà algebroide $M(\mathfrak{p}, j) d i(\mathfrak{p}, j)$ consiste di componenti irriducibili $M\left(\mathfrak{q}_{\lambda}\right), \lambda=1,2, \ldots, r$ con la propriet⿳亠丷厂 $\mathfrak{q}_{\lambda} \cap \mathbb{p}_{k}=\mathfrak{i}$. La dimensione di ogni ideale primo $\mathfrak{q}_{2}$ coincide con la dimensione $d i \mathfrak{i}$.

Per il teorema 35 (3) i fattori irridncibili della funzione norma $\Phi(\overline{\mathbf{p}})$ di $\overline{\mathfrak{p}}$ fissano delle componenti irriducibili di $M(\mathfrak{p}, j)$ con la proprietà richiesta. Rimane ancora da dimostrare che $M(\mathfrak{p}, j)$ non ne possiede altre. Sia $\mathfrak{q}$ un punto di $M(\mathfrak{p}, j)$ e $\mathfrak{q} \cap \mathbb{P}_{k}=w$; sia inoltre $\overline{\mathfrak{q}}=\mathfrak{q} \cap \overline{\mathbb{P}}_{k, n-k}$. Essendo $\overline{\mathfrak{p}} \subset \overline{\mathfrak{q}}$, per i teoremi 24 e $14 \overline{\mathfrak{q}}$ possiede una base in $\overline{\mathbb{p}}_{k, n-k}$; l'estensione di $\overline{\mathfrak{q}}$ in $\mathbb{p}$, coincide percio con $\mathfrak{q}$. $\mathrm{Da}(\mathfrak{p}, j) \subset \mathfrak{q}$ segue $\mathfrak{i} \subset w$. Consideriamo uno accanto all'altro gli omomorfismi delle classi resto $\mathbb{D}_{k} \rightarrow \mathbb{D}^{*}=\mathbb{D}_{k} / \mathbf{i} \in \mathbb{p}_{k} \rightarrow \mathbb{D}^{* *}=\mathbb{P}_{k} / w$, e le loro estensioni negli anelli di polinomi. Il secondo si puo eseguire in due tempi. Poichè per il primo $w$ va nell' ideale primo $w^{*}$ (cfr. 12.6. (a)), $\mathbb{P}^{* *}$ è isomorfo a $\mathbb{D}^{*} / w^{*}$. Se ora le immagini di $\Phi(\overline{\mathfrak{p}})$ sono i polinomi $\Phi^{*}(\overline{\mathfrak{p}}, \mathfrak{i})$ risp. $\Phi^{* *}(\overline{\mathfrak{p}}, w)$. allora $\Phi^{* *}$ nasce da $\Phi^{*}$ mediante l'omomorfismo $\mathbb{p}^{*} \rightarrow \mathbb{p}^{* *}$. Ora per il teorema $35 \Phi^{*}(\overrightarrow{\mathfrak{p}}, \mathfrak{i})$ è il prodotto delle funzioni norma : $\Phi^{*}(\mathfrak{q} ; *), \lambda=1,2, \ldots, r$ :

$$
\Phi^{*}(\overline{\mathfrak{p}}, \mathfrak{i})=\prod_{\lambda=1}^{r} \Phi^{*}\left(\mathfrak{q}_{\lambda}^{*}\right)
$$

Applicando l'omomorfismo $\mathbb{P}^{*} \rightarrow \mathbb{P}^{* *}$ ne viene:

$$
\Phi^{* *}(\overline{\mathbf{p}}, w)=\prod_{\lambda=1}^{r} \Phi^{* *}\left(\mathbf{q}_{2}^{*}, w^{*}\right)
$$

Indichiamo con $\mathfrak{q}^{* *}$ l'immagine di $\overrightarrow{\mathfrak{q}}$. La funzione norma $\Phi^{* *}\left(\mathfrak{q}^{* *}\right)$ per il teorema 35 è allora divisore di $\Phi^{* *}(\overline{\mathfrak{p}}, w)$ e divide percio uno dei fattori del prodotto a secondo membro della (2), sia p. es. $\Phi^{* * *}\left(\mathfrak{q}_{1}{ }^{*}, w^{*}\right)$. E allora $\dot{\mathbf{\theta}}$ $\mathfrak{q}_{1}{ }^{*} \subset \mathfrak{q}^{*}$ per il teorema 34, che noi applichiamo a $\mathbb{P}^{*} \rightarrow \mathbb{P}^{* *}$. Essendo $\mathfrak{i} \subset w \subset \overline{\mathbf{q}}$, segue $\overline{\mathfrak{q}}_{1} \subset \overline{\mathfrak{q}}$ e per le estensioni in $\mathbb{P}_{n} \mathfrak{q}_{1} \subset \mathfrak{q}$, il che significa che $M\left(\mathfrak{q}_{1}\right)$ con. tiene il punto $\mathfrak{q}$. Tutti $i$ punti di $M(\mathfrak{p}, j)$ appartengono quindi alle $M\left(\mathfrak{q}_{\lambda}\right)$, $\lambda=1,2, \ldots, r$.

13.2. Il teorema 36 è un approfondimento di uno risultato in 9.7, appli. cato allo stato di cose di colà esso porge il risultato: Ogni componente primaria isolata dell'ideale $\mathfrak{q}=\left(\mathfrak{p}, y_{k}\right)$ ha la dimensione $k-1$. Come il teorema 36 prosegue nella direzione stabilita il :

Teonema 37.

Sia $\mathbb{P}$ un corpo con infiniti elementi; e sia $\mathfrak{p}$ un ideale primo di dimen. sione $k \geqq 1$ nell' anello di serie di potenze $\mathbb{P}_{n}$. Sia inoltre $f$ una serie irriduci. bile di $\mathbb{\mathbb { P }}_{\text {i }}$ diversa da una unitì di $\overrightarrow{\mathbb{D}}_{n}$. Se f non appartiene a $\mathbf{p}$ ogni compo. nente primaria isolata dell' ideale $(\mathfrak{p}, f)$ ha la dimensione $k-1$. 
(a) Dimostriamo dapprima un caso particolare di questo teorema, supponendo ohe $f$ sia una forma lineare di $x_{1}, x_{2}, \ldots, x_{n}$. Eseguendo una If-trasformazione di coordinate si può ottenere che $f=x_{1}$. Le segnenti considerazioni riposano sopra un complemento del teorema 18.

\section{LEMMa 1.}

Sia $\mathbb{D}$ un corpo con infiniti elementi ; e sia $F(x)$ ana serie dell'anello $\mathbb{D}_{n}$, non divisibile per $x_{1}$. Allora: $F$, mediante una $\mathbb{P}$-trasformazione di coordinate:

$$
\begin{aligned}
& y_{1}=x_{1} \\
& y_{i}=a_{i 2} x_{2}+a_{i 8} x_{3}+\ldots+a_{i n} x_{12}
\end{aligned}
$$

$$
i=2,3, \ldots, n \text {, }
$$

si può ricondurre ad una serie $F^{*}$ che è privilegiata rispetto ad $y_{n}$.

Sostituendo $x_{1}=0$ in $F$ si ottiene una serie $F_{1}\left(x_{2}, x_{3}, \ldots, x_{n}\right)$ che, per la nostra ipotesi, non si annulla; ed è $F=F_{1}+x_{1} F_{2}$ con $F_{2} \in \mathbb{P}_{n}$. Per il teorema 18 esiste una trasformazione di coordinate $L_{1}$ delle indeterminate $x_{2}, x_{3}, \ldots, x_{n}$, tale che $F_{1}^{*}$ divenga privilegiata rispetto ad $y_{n}$, ciò̀ $F_{1}^{*}$ contenga un addendo $\alpha y_{n}^{*}$ con $\alpha \in \mathbb{P}$. Ampliando $L_{1}$ con $y_{1}=x_{1}$, si ottiene una $\mathbb{P}$-trasformazione di coordinate del tipo 13(1). Inoltre è $F^{*}=F_{1}^{*}+y_{1} F_{2}{ }^{*}$, ed anche in $F^{*}$ si presenta l'addendo $\alpha y_{n}{ }^{s}$; ciò̀ $F^{*}$ è privilegiata rispetto ad $y_{n}$.

(b) Poggiando su questo lemma il teorema 20 si lascia così completare:

\section{LAMMA 2.}

Sia un corpo con infiniti elementi; e sia a un ideale primo nell'anello delle serie di potenze $\mathbb{P}_{n}, \mathbf{a} \neq \mathbb{P}_{n}$. Se $x_{1}$ non è elemento di $\mathfrak{a}$, si può scegliere la D-trasformazione di coordinate che riconduce $a$ ad un ideale con una base di polinomi, in modo ohe essa sia del tipo $13(1)$.

Per dimostrare ciò osserviamo dapprima che per un ideale primo $\mathfrak{p} d a$ $\mathfrak{p} \subset\left(x_{1}\right)$ segue che $\mathfrak{p}=\left(x_{1}\right)$. Se infatti $f \in \mathfrak{p}$ risulta $f=x_{1}{ }^{\alpha} f^{\prime}$ con $\alpha>0$ dove $f^{\prime}$ non fa parte di $\left(x_{1}\right)$ e quindi neppure di $p$. Ma poichè $p$ è primo, ne segue $x_{1} \in \mathfrak{p}$ e perciò $\mathfrak{p}=\left(x_{1}\right)$.

Per dimostrare il lemma 2 seguiamo la dimostrazione del teorema 20. Si supponga che la $\mathbb{P}$-trasformazione di coordinate $L_{r}$ abbia la forma $13(1)$; $a^{(\cdot)}$ non contiene allora $\xi_{1}^{(r)}$. Questa indeterminata non appartiene perciò all'ideale primo $\mathbb{P}_{r} \xi^{(r)} \cap \mathfrak{a}^{(r)}$. Nel caso quindi che questa intersezione non sia l'ideale (0), per l'osservazione precedente si può scegliere in essa l'elemento $f\left(\xi^{(r)}\right)_{r}$ in modo che non sia divisibile per $\xi_{1}^{(r)}$. (Si osservi, che a è ideale primo). In conseguenza, per il lemma 1 , si può scegliere la $\mathbb{P}$-trastormazione di coordinate $L_{r+1}^{\prime}$ nella particolare forma 13(1); anche $L_{r+1}$ allora ha la medesima forma. Le rimanenti considerazioni nella dimostrazione del teorema 20 rimangono invariate. 
(c) Non appartenendo dunque $f=x_{1}$ all'ideale primo $\mathfrak{p}$, si può determinare una $\mathbb{P}$-trasformazione di coordinate $13(1)$ tale che $\mathfrak{p}$ abbia una base di polinomi in $\overline{\mathbb{D}}_{k, n-k}$; essa muta $f$ in $y_{1}$. A $\mathfrak{p}$ e $\mathfrak{i}=\left(y_{1}\right)$ si può applicare il teorema 36 , essendo $y_{1} \in \mathbb{P}_{k}$. La varietà algebroide di $\left(\mathfrak{p}, y_{1}\right)$ consiste di componenti irriducibili di dimensione $k-1$. Con ciò è dimostrato il teorema 37 nel caso particolare di una forma lineare $f$.

(d) Riconduciamo ora il caso generale al caso particolare già dimostrato, mediante il seguente artificio:

Sia $x_{n+1}$ una nuova indeterminata. Ogni elemento $f$ dell'anello delle serie di potenze $\mathbb{P}_{n+1}=\mathbb{P}_{n}\left[\left[x_{n+1}\right]\right]$ è univocamente rappresentabile nella forma $f=f_{0}+x_{n+1} f_{1}$ con $f_{0} \in \mathbb{P}_{n}$ ed $f_{1} \in \mathbb{P}_{n+1}$. Consideriamo in $\mathbb{P}_{n+1}$ 'ideale $\mathfrak{p}^{(1)}=\left(\mathfrak{p}, x_{n+1}\right)$.

(1) $\mathfrak{p}^{(1)}$ è un ideale primo di $\mathbb{P}_{n+1}$.

Se infatti $f \cdot g \in \mathfrak{p}^{(1)}$ con $f, g \in \mathbb{P}_{n+1}$, si ha $f=f_{0}+x_{n+1} \cdot f_{1}$ e $g=g_{0}+x_{n+1} g_{1}$ con $f_{0}, g_{0} \in \mathbb{P}_{n}$ e $f_{1}, g_{1} \in \mathbb{P}_{n+1}$. Di qui segue $f_{0} \cdot g_{0}+x_{n+1} h \in \mathfrak{p}^{(1)}$ con $h \in \mathbb{P}_{n+1}$. Ora deve essere $f_{0} \cdot g_{0} \in \mathfrak{p} ;$ e poichè $\mathfrak{p}$ è un ideale primo ne viene ohe 0 $f_{0} \in \mathfrak{p}$ oppure $g_{0} \in \mathfrak{p}$. Nel primo caso è $f \in \mathfrak{p}^{(1)}$, nel secondo $g \in \mathfrak{p}^{(1)}$.

(2) Le dimensioni di $\mathfrak{p}$ e $\mathfrak{p}^{(1)}$ coincidono. Avendo $\mathfrak{p}$ una base di polinomi nell' anello $\overline{\mathbb{D}}_{k, n-k}=\mathbb{p}_{k}\left[y_{k+1}, y_{k+2}, \ldots, y_{n}\right], \mathfrak{p}^{(1)}$ ha una base di polinomi nell' anello $\overline{\mathbb{D}}_{k, n+1-k}=\mathbb{D}_{k}\left[y_{k+1}, y_{k+2}, \ldots, y_{n}, y_{n+1}\right]$ con $y_{n+1}=x_{n+1}$.

I precedenti risultati si possono invertire nella seguente forma:

(3) Se $\mathfrak{q}^{(1)}$ \& un ideale primo in $\mathbb{P}_{n+1}$, diverso dall' ideale principale $\left(x_{n+1}\right)$, ed è $x_{n+1} \in \mathfrak{q}^{(1)}$, allora $\mathfrak{q}=\mathfrak{q}^{(1)} \cap \mathbb{p}_{n}$ e un ideale primo di $\mathbb{P}_{n}$ avente la medesima dimensione di $\mathfrak{q}^{(1)}$.

Se $F_{1}, F_{2}, \ldots, F_{x}$ ə una base di $\mathfrak{q}^{(1)}$ ed è $F_{i}=f_{i}+x_{n+1} F_{i}^{\prime}$ con $f_{i} \in \mathbb{P}_{n}$, allora $f_{1}, f_{2}, \ldots, f_{a}, x_{n+1}$ costituiscono una base di $\mathbf{q}^{(1)}$. $\mathbf{E}$ allora $\mathbf{q}=\left(f_{1}, f_{2}\right.$, .,,$\left.f_{x}\right)$ e $\mathfrak{q}^{(1)}=\left(\mathfrak{q}, x_{n+1}\right)$. Da 1.3. (b) e (2) seguono ora le nostre affermazioni.

(e) Sottoponiamo l'anello delle serie di potenze $\mathbb{P}_{n+1}$ alla trastormazione formalmente pseudoconforme:

$$
\begin{array}{ll}
z_{i}=x_{i}, & i=1,2, \ldots, n, \\
z_{n+1}=x_{n+1}+f\left(x_{1}, x_{2}, \ldots, x_{n}\right) . &
\end{array}
$$

Per essa l'ideale primo $\mathfrak{p}^{(1)}=\left(\mathfrak{p}, x_{n+1}\right)$ si muta in un ideale primo $\mathfrak{p}^{*}$ della medesima dimensione $k$, cfr. il teorema 9. Costruiamo l'ideale $\left(\mathfrak{p}^{*}, z_{n+1}\right) \cdot z_{n+1}$ appartiene a $\mathfrak{p}^{*}$ se $\theta$ soltanto se $f \in \mathfrak{p}$. Fatta astrazione da questo chso eccezionale, la varieta algebroide $M\left(\mathfrak{p}^{*}, z_{n+1}\right)$ per la validità del nostro teorema già dimostrata nel caso particolare di cui in (c), consiste di componenti irridacibili $M\left(\mathfrak{q}_{i}^{*}\right), i=1,2, \ldots, r$, di dimensione $k-1$. Eseguendo in senso 
inverso la trasformazione (1), da $\mathfrak{q}_{i}^{*}$ si ottenga l'ideale primo ( $k-1$-dimensionale) $\mathfrak{q}_{i}^{(1)}($ per $i=1,2, \ldots, r)$. Da $\left(\mathfrak{p}^{*}, z_{n+1}\right) \subset \mathfrak{q}^{*}$ segue: $\left(\mathfrak{p}, x_{n+1}, x_{n+1}+f\right) \subset \mathfrak{q}_{i}^{(1)}$ e quindi $(\mathfrak{p}, f) \subset \mathfrak{q}_{i}=\mathfrak{q}_{i}^{(1)} \cap \mathbb{P}_{n}$. Naturalmente nessun ideale $\mathfrak{q}_{i}^{(1)}$ può coincidere con l'ideale principale $\left(x_{n+1}\right)$. Come è stato dimostrato in $(d)$, gli ideali $\mathfrak{q}_{i}$ sono primi e hanno la dimensione $k-1$. Segue inoltre:

$$
M\left(\mathfrak{q}_{1}\right) \cup M\left(\mathfrak{q}_{2}\right) \cup \ldots \cup M\left(\mathfrak{q}_{r}\right) \subset M(\mathfrak{p}, f)
$$

Viceversa sia $\mathfrak{q}$ un punto di $M(\mathfrak{p}, f)$. Allora è $(\mathfrak{p}, f) \subset \mathfrak{q}$ e in $\mathbb{P}_{n+1}$ :

$$
\left(\mathfrak{p}, x_{n+1}, f\right) \subset\left(\mathfrak{q}, x_{n+1}\right)=\mathfrak{q}^{(1)}
$$

Di qui si ha ancora:

$$
\left(\mathfrak{p}, x_{n+1}, x_{n+1}+f\right) \subset \mathbf{q}^{(1)}
$$

Dopo la trasformazione (1) cio significa che;

$$
\left(\mathbf{p}^{*}, z_{n+1}\right) \subset \mathbf{q}^{*}
$$

Per quanto visto in $(d) \mathfrak{q}^{(1)}$, e perciò $\mathfrak{q}^{*}$, è un ideale primo; e allora $\mathfrak{q}^{*}$ è un punto di una delle varietà algebroidi $M\left(\mathfrak{q}_{i}^{*}\right)$, sia ad es. di $M\left(\mathfrak{q}^{*}\right)$. Da $\mathfrak{q}_{1}{ }^{*} \subset \mathfrak{q}^{*}$ viene ora $\mathfrak{q}_{1}{ }^{\langle 1\rangle} \subset \mathfrak{q}^{(1)}$ e inoltre per intersezione con $\mathbb{P}_{n}: \mathfrak{q}_{1} \subset \mathfrak{q}$, cioè $\mathfrak{q}$ appartiene ad $M\left(\mathrm{q}_{1}\right)$. Da cio e da (2) segue infine l'affermazione del teorema 37 :

$$
M(\mathfrak{p}, f)=M\left(\mathfrak{q}_{1}\right) \cup M\left(\mathfrak{q}_{2}\right) \cup \ldots \cup M\left(\mathfrak{q}_{r}\right)
$$

13.3. Come complemento al teorema 37 stabiliamo un procedimento per la determinazione delle componenti irriducibili di $M(p, f)$, $f$ una non-unità di $\Phi_{n}$.

Quando $f$ non appartiene a $p$ esiste, per il teorema 24, corollario 3, un polinomio irriducibile in $f$ :

$$
f^{m}+c_{1} f^{m-1}+\cdots+c_{m-1} f+a
$$

con coefficienti in $D_{k}$, appartenente a $\mathfrak{p}$. a non appartiene a $\mathfrak{p} \theta$ si chiama la norma di $f$ rispetto a $p$. Siccome $f$ non è una unità di $\mathbb{P}_{n}$, la norma non è una unità di $\mathbb{Q}_{k}$, cfr. 9.2. La varietà algebroide $M(p, a)$ sia formata dalle componenti irriducibili, di dimensione $k-1, M\left(\mathfrak{p}_{i}\right), i=1,2, \ldots, r, f$ potrebbe stare in $\mathfrak{p}_{1}, \mathfrak{p}_{2}, \ldots, \mathfrak{p}$, $\Theta$ non in $\mathfrak{p}_{s+1}, \mathfrak{p}_{s+2}, \ldots, \mathfrak{p}_{r}$ e allora $\grave{e}$ :

$$
M(\mathfrak{p}, f)=M\left(\mathfrak{p}_{1}\right) \cup M\left(\mathfrak{p}_{2}\right) \cup \ldots \cup M\left(\mathfrak{p}_{k}\right) .
$$


Per dimostrarlo, si osservi che da $(\mathfrak{p}, a) \subset(\mathfrak{p}, f)$ segue $M(\mathfrak{p}, f) \subset M(\mathfrak{p}, a)$. $\mathrm{E}$ siccome anche $M(\mathfrak{p}, f)$ è l'unione di varietà algebroidi irriducibili di dimensione $k-1$, \& vero quanto affermato.

Per determinare $M(\mathfrak{p}, f)$, si consideri la norma $a$ di $f$ rispetto a $\mathfrak{p}$ e si determini $M(\mathfrak{p}, a)$. Dopo ciò $M(\mathfrak{p}, f)$ consiste di quelle componenti di $M(\mathfrak{p}, a)$ su cui si annulla $f$, efr. [15], pag. 123.

13.4. Una ulteriore applicazione del teorema 37 in relazione con una nuova caratterizzazione invariante della dimensione di un ideale.

Teorema 38.

Sia IP un corpo con infiniti elementi; e sia a un ideale nell'anello delle serie di potenze $\mathbb{D}_{n}$. La dimensione $k$ di a è caratterizzata dalle due seguenti proprietà :

(1) Esistono in $\mathbb{P}_{n} k$ serie $f_{1}, f_{2}, \ldots, f_{k}$, che non sono unità di $\mathbb{P}_{n}$, tali che l'ideale ( $\mathrm{a}, f_{1}, f_{2}, \ldots, f_{k}$ ) ̀̀ un ideale primario dell'ideale massimo $\mathrm{m}=\left(x_{1}, x_{2}, \ldots, x_{m}\right) d i \mathbb{P}_{n}$.

(2) Non esistono meno di $k$ serie in $\mathbb{P}_{n}$ con la medesima proprietà.

Con la terminologia delle varietà algebroidi il teorema 38 si enuncia come segue:

La varietà algebroide $M(\mathfrak{a})$ ha la dimensione $k$, quando $k$ è il più piccolo numero tale che per $k$ serie $f_{1}, f_{2}, \ldots, f_{k}$ che non siano unità, l'intersezione $M(a) \cap M\left(f_{1}\right) \cap M\left(f_{2}\right) \cap \ldots \cap M\left(f_{k}\right)$ coincide col punto $m=\left(x_{1}, x_{2}, \ldots, x_{n}\right)$.

Enunciato il teorema in questa forma, si riconosce che è sufficiente dimostrarlo per un ideale primo $p$. Sia $p$ a $k$ dimensioni ed abbia una base di polinomi nell'anello $\overline{\mathbb{D}}_{k, n-k}$. Si ponga $f_{1}=y_{1}, f_{2}=y_{2}, \ldots, f_{k}=y_{k}$. Dato che $\mathfrak{p}$ contiene il polinomio $\bar{p}_{k+j}, j=1,2, \ldots, n-k$, delle potenze di $y_{k+1}$, $y_{k+2}, \ldots, y_{n}$ giacciono in $\left(\mathfrak{p}, y_{1}, y_{2}, \ldots, y_{k}\right)$; perciò questo ideale $\ominus$ un ideale primario di m. D'altra parte segue dal teorema 37 che $M\left(p, f_{1}, f_{2}, \ldots, f_{v}\right)$ per arbitrarie serie $f_{1}, f_{2}, \ldots, f_{v}$ nel easo $\vee \leq k-1$ contiene soltanto componenti irriducibili di dimensione $\geq k-v \geq 1$.

\section{§ 14. - Le falde di una varietà algebrica.}

14.1. L'applicazione della teoria delle serie di potenze alla geometria algebrica si basa sopra i teoremi di estensione del $\S 8$, efr, [4], pag. 147, e sopra la teoria della funzione norma.

Sia $\mathbb{P}$ un corpo. Nell'anello di polinomi $\mathbb{P}_{n}{ }^{*}=\mathbb{P}\left[x_{1}, x_{2}, \ldots, x_{n}\right]$ sia $\mathfrak{a}^{*}$ un ideale, il quale definisca una varieta algebrica $M^{*}\left(\mathfrak{a}^{*}\right)$. Il nostro scopo $\&$ lo studio delle proprietà locali di $M^{*}\left(\mathfrak{a}^{*}\right)$, cioè delle proprietà di $M^{*}\left(\mathfrak{a}^{*}\right)$ nel- 
l'intorno di un punto. Senza venir meno alla generalità si può scegliere questo punto come origine dello spazio $\left\{x_{1}, x_{2}, \ldots, x_{n}\right\}$, poichè ogni altro punto $\left\{x_{1}^{0}, x_{2}^{0}, \ldots, x_{n}^{0}\right\}$ si può ricondurre nel punto 0 mediante la trasformazione :

$$
x_{i}^{\prime}=x_{i}-x_{i}{ }^{0}, \quad i=1,2, \ldots, n .
$$

Indicando con $x^{*}$ Irdeale $\left(x_{1}, x_{2}, \ldots, x_{n}\right), a^{*}$ è contenuto in $x^{*}$. Per il teorema $22 \mathfrak{a}^{*}$ è intersezione di ideali $\mathfrak{b}^{*}$ e $\mathfrak{C}^{*}$, dove $\mathfrak{b}^{*}$ non ha alcuna com. ponente primaria $\mathfrak{q}^{*}$ per cui $\left(\mathfrak{q}^{*}, x^{*}\right)=\mathbb{p}_{n}{ }^{*}$ ed è inoltre $\left(\mathfrak{C}^{*}, x^{*}\right)=\mathbb{p}_{n}{ }^{*}$. Se ora $\mathfrak{a}=\mathbb{P}_{n} \cdot \mathfrak{a}^{*}$ è l'ideale estensione di $\mathfrak{a}^{*}$ nell'anello delle serie (formale) di potenze $\mathbb{P}_{n}$, risulta, per il teorema 22 :

$$
\mathfrak{a} \cap \mathbb{P}_{n}^{*}=\mathfrak{b}^{*}
$$

14.2. Consideriamo più da vicino l'ideale $\mathfrak{b}^{*}$. Considerazioni geometriche suggeriscono di non prendere in esame quelle componenti di $M^{*}\left(a^{*}\right)$ che non contengono il punto 0 ; esse non hanno interesse per le nostre considerazioni locali. Per l'estensione $\mathfrak{b}=\mathbb{p}_{n} \cdot \mathfrak{b}^{*}$ di $\mathfrak{b}^{*}$ in $\mathbb{p}_{n}$ vale allora la relazione:

$$
\mathfrak{b} \cap \mathbb{P}_{n}^{*}=\mathfrak{b}^{*} \text {. }
$$

Sia $M(b)$ la varietà algebroide di $\mathfrak{b}$; pensiamo di decomporla in componenti irriducibili, ofr. 10.4:

$$
M(\mathbf{b})=M\left(\boldsymbol{3}_{1}\right) \cup M\left(\mathfrak{z}_{2}\right) \cup \ldots \cup M\left(\boldsymbol{3}_{r}\right) ;
$$

qui è $3_{i}$ (per $i=1,2, \ldots, r$ ) un ideale primo di $\mathbb{P}_{n}$. Le varietà algebroidi $M\left(3_{i}\right)$ si chiamano le falde della varietà algebrica $M^{*}\left(\mathfrak{a}^{*}\right)$ nel punto 0 .

14.3. Per scoprire proprietà più riposte delle falde di una varietà algebrica, $\doteq$ necessario avere di esse una migliore conoscenza. Noi supponiamo che sia un corpo con infiniti elementi; e per semplicità supponiamo pure che sia algebricamente ohiuso. Questa ipotesi si puo in realta evitare, pen. sando come corpo "crescente», che durante un calcolo si può ampliare algebricamente quante volte occorre, cfr. [20], pag. 105. Assumiamo inoltre un ideale primo $\mathfrak{p}^{*}$ di dimensione $k$, alla base delle nostre considerazioni. Con queste ipotesi vale il teorema di normalizzazione, [4], pag. 134. Esiste una p-trasformazione di coordinate

$$
(y)_{n}=L(x)_{n}
$$

tale che $\mathfrak{p}^{*}$ contenga un polinomio irriducibile in $y_{k+j}($ per $i=1,2, \ldots, n-k)$ con coefficienti in $\mathbb{P}_{k}{ }^{*}=\mathbb{P}\left[y_{1}, y_{2}, \ldots, y_{k}\right]$ e coefficiente del termine di grado 
massimo uguale 1 , ad mentre risulti $\mathfrak{p}^{*} \cap \mathbb{D}_{k} *=(0)$. Dopo questa normalizzazione, sulla varietà $M^{*}\left(\mathfrak{p}^{*}\right)$ le quantità $y_{k+j}$ sono algebriche intere sopra $\mathbb{P}_{k}{ }^{*}$. Esiste allora la funzione norma (cfr. 12.1)

$$
\Phi\left(\mathbf{p}^{*}\right)=\Phi\left(z, u_{k+1}, u_{k+2}, \ldots, u_{n},(y)_{k}\right)
$$

che $\dot{e}$ il polinomio irriducibile dell'anello $\mathbb{P}\left[y_{1}, y_{2}, \ldots, y_{k}, u_{k+1}, u_{k+2}, \ldots, u_{n}, z\right]$ avente lo zero:

$$
\zeta=u_{k+1} \eta_{k+1}+u_{k+1} \eta_{k+2}+\ldots+u_{n} \eta_{n},
$$

dove $\eta_{k+j}$ indica la classe resto di $y_{k+j} \bmod \mathfrak{p}^{*}$ nell' anello $\mathbb{P}_{n}^{*} / \mathfrak{p}^{*}$.

14.4. Se applichiamo il teorema 34 all'ideale $\mathfrak{p}^{*}$ con $R=\mathbb{p}_{k}{ }^{*}$, essendo $\mathfrak{i}=\left(y_{1}-\bar{y}_{1}, y_{2}-\bar{y}_{2}, \ldots, y_{k}-\bar{y}_{k}\right)$, e supponiamo che ogni ideale primo di $\mathbb{p}_{n}{ }^{*} \mathrm{di}$ dimensione 0 [sopra il corpo algebricamente chiuso (P) abbia la forma $\left(y_{1}-\bar{y}_{1}, y_{2}-\bar{y}_{2}, \ldots, y_{n}-\bar{y}_{n}\right)$, allora otteniamo i risultati seguenti :

(a) Essendo $(\bar{y})_{n}$ un punto di $M^{*}\left(\mathfrak{p}^{*}\right)$,

$$
z-u_{k+1} \bar{y}_{k+1}-u_{k+2} \bar{y}_{k+2}-\ldots-u_{n} \bar{y}_{n}
$$

risulta divisore del polinomio:

$$
\Phi\left(z, u_{k+1}, u_{k+2}, \ldots, u_{n},(\bar{y})_{k}\right) .
$$

(b) Per un arbitrario gruppo di valori $(\bar{y})_{k}$ il polinomio $14(8)$ si decompone nel prodotto di fattori lineari $14(7)$, in modo che $\bar{y}_{k+1}, \bar{y}_{k+2}, \ldots, \bar{y}_{n}$ sono indipendenti dalle $u_{k+j}$ e $(\bar{y})_{n}$ è un punto di $M^{*}\left(\mathfrak{p}^{*}\right)$.

Introducendo in particolare in $\Phi\left(\mathfrak{p}^{*}\right)$ i valori $y_{1}=0, y_{2}=0, \ldots, y_{k}=0 \mathrm{si}$ ottiene un prodotto di potenze di fattori lineari:

$$
z-u_{k+1} y_{k+1}^{(2)}-u_{k+2} y_{k+2}^{(\lambda)}-\ldots-u_{n} y_{n}^{(\lambda)} .
$$

I punti $P_{\lambda}=\left\{(0)_{k}, y_{k+1}^{(\lambda)}, y_{k+2}^{(\lambda)}, \ldots, y_{n}^{(\lambda)}\right\}$ stanno su $M^{*}\left(\mathfrak{p}^{*}\right)$. Tra questi fattori lineari compare anche $z$, perchè il punto 0 appartiene ad $M^{*}\left(\mathfrak{p}^{*}\right)$.

14.5. Il seguente teorema porge un metodo per la determinazione delie falde di una varietà algebrica per mezzo della funzione norma.

Teorema 39.

Sia $\mathbb{D}$ un corpo algebricamente chiuso (cfr. 14.3.); e sia $\mathfrak{p}^{*}$ un ideale primo $a k$ dimensioni nell'anello di polinomi $\mathbb{P}_{n}{ }^{*}=\mathbb{P}\left[x_{1}, x_{2}, \ldots, x_{n}\right]$. Il punto 0 di coordinate $x_{1}=0, x_{2}=0, \ldots, x_{n}=0$ appartenga alla varietà algebrica $M^{*}\left(\mathfrak{p}^{*}\right)$ di $\mathfrak{p}^{*}$. Dopo la $\mathbb{P}$-trasformazione di coordinate $14(\overline{0}), \mathfrak{p}^{*}$ abbia una funzione 
norma $\Phi\left(\mathfrak{p}^{*}\right)=\Phi\left(z, u_{k+1}, u_{k+2}, \ldots, u_{n},(y)_{k}\right)$, che $\grave{e}$ un polinomio dell' anello $\mathbb{D}\left[y_{1}, y_{2}, \ldots, y_{k}, u_{k+1}, u_{k+2}, \ldots, u_{n}, z\right]$, cfr. 14.3. Indicando con $\mathbb{D}_{k}$ l'anello delle serie di polenze formale $\mathbb{P}\left[\left[y_{1}, y_{2}, \ldots, y_{k}\right]\right], \Phi\left(\mathfrak{p}^{*}\right)$ in quanto polinomio dell'anello $\mathbb{P}_{k}\left[u_{k+1}, u_{k+2}, \ldots, u_{n}, z\right]$ sarc̀ in generale riducibile. Sia

$$
\Phi\left(\mathfrak{p}^{*}\right)=\prod_{p=1}^{m} \Psi_{k}^{*}
$$

la sua decomposizione nei fattori irriducibili in questo anello. Per $\mu=1,2$, $\ldots, m, \Psi_{\mu}$ è un polinomio in $z$ con coefficiente del termine di grado massimo ugwale ad.1, $i$ cui ulleriori coefficienti appartengono all'anello $\mathbb{D}_{k}\left[u_{k+1}, u_{k+2}\right.$, $\left.\ldots, u_{n}\right]$.

(a) Ogni polinomio $\Psi_{1 .}$ è la funzione norma di un ideale primo $\overline{\mathfrak{q}}_{\mu}$ del$l$ 'anello di polinomi $\overline{\mathbb{D}}_{k, n-k}=\mathbb{P}_{k}\left[y_{k+1}, y_{k+2}, \ldots, y_{n}\right]$.

(b) Il fattore $\Psi_{\lambda}$ abbia la proprietà seguente:

Mediante la sostituzione

$$
y_{1}=0, y_{2}=0, \ldots, y_{k}=0
$$

il polinomio $\Psi_{\lambda}$ si mula in una potenza di z. Allora l'ideale $\mathfrak{q}_{\lambda}$ estensione di $\overline{\mathfrak{q}}_{\lambda}$ nell'anello delle serie di potenze $\mathbb{D}_{n}$ è un ideale primo di $\mathbb{P}_{n}$. Vale la relazione

$$
\overline{\mathfrak{q}}_{\lambda}=\mathfrak{q}_{\lambda} \cap \overline{\mathbb{p}}_{k, n-k} .
$$

(c) Siano $\Psi_{\lambda}$ (per $\left.\lambda=\mu_{1}, \mu_{2}, \ldots, \mu_{h}\right)$ quei fattori in 14(10) che, con la sostituzione 14(11) si riducono a potenze di z. Dato che, per quanto visto in (b), l'ideale primo $\mathfrak{q}_{\lambda}$ appartiene a $\Psi_{\lambda}$, le varietà algebroidi $M\left(\mathfrak{q}_{\lambda}\right)$ sono le falde di $M^{*}\left(\mathfrak{p}^{*}\right)$ nel punto 0 ; in altri termini: Indicando con $\mathfrak{p}$ l'ideale esteso $\mathbb{P}_{n} \cdot \mathfrak{p}^{*}$ di $\mathfrak{p}^{*}$ nell'anello delle serie di potenze $\mathbb{P}_{n}$, la varietà algebroide $M(\mathfrak{p})$ è l'unione delle varietà algebroidi irriducibili $M\left(\mathfrak{q}_{\lambda}\right)$, per $\lambda=\mu_{1}, \mu_{2}, \ldots, \mu_{n}$.

(a) Sia $\zeta^{*}$ uno zero di $\Psi_{\mu}$ in un ampliamento algebrico del corpo quoziente di $\mathbb{p}_{k}\left[u_{k+1}, \ldots, u_{n}\right]$, opportunamente scelto. Dalla proprietà risultante della funzione norma 12.2 segue, come nella dimostrazione del teorema 34 , che

$$
\zeta^{*}=u_{k+1} \eta_{k+1}^{*}+u_{k+2} \eta_{k+2}^{*}+\ldots+u_{n} \eta_{n}^{*}
$$

dove le quantità $\eta_{k+i}^{*}$ sono indipendenti dalle $u_{k+j}$, e il sistema $\eta^{*}=\left\{\eta_{k+1}^{*}, \eta_{k+2}^{*}, \ldots, \eta_{n}^{*}\right\}$ è uno zero di $\mathfrak{p}^{*}$. La totalità di tatti i polinomi dell' anello $\overline{\mathrm{p}}_{k, n-k}$, che si annullano in $\eta^{*}$, è un ideale primo che indichiamo con $\overline{\mathfrak{q}_{\mu}} ;$ cio posto si ha

$$
\widetilde{\mathbb{P}}_{k, n-k} \cdot \mathfrak{p}^{*} \subset \overline{\mathfrak{q}}_{\mu}
$$


$\Psi_{\mu}$ in quanto polinomio irriducibile con lo zero (1) è la funzione norma di $\overline{\mathfrak{q}}_{\mu}$; con ciò è dimostrato il punto (a).

(b) Dimostriamo che $\overline{\mathbf{q}}$. soddisfa alle ipotesi del teorema 23.

(1) Sotto le ipotesi (b) sopra $\Psi_{\lambda}$, l'ideale di annullamento $\overline{\mathbf{a}}_{\lambda}$ di $\Psi_{\lambda}$ contiene per ogni $i=1,2, \ldots, n-k$ un polinomio $\bar{p}_{k+j}\left(y_{k+j} \mid(y)_{k}\right)$ dell'anello $\left.\mathbb{D}_{k} \mid y_{k+j}\right]$, cfr. 12.4. (b); e questo polinomio si riduce, dopo la sostituzione $14(11)$, ad una potenza di $y_{k+j}$ e quindi è privilegiato sopra $\mathbb{P}_{k}$. Poichè $\overline{\mathfrak{a}}_{\lambda} \subset \overline{\mathfrak{q}}_{\lambda}$, le $\bar{p}_{k+j}$ appartengono anche a $\overline{\mathfrak{q}}_{\lambda}$.

(2) Dall'ipotesi sopra $\Psi_{\lambda}$ inoltre consegue che $\overline{\mathfrak{a}}_{\lambda}$ è contenuto nell'ideale $\left(y_{1}, y_{2}, \ldots, y_{n}\right)$ di $\overline{\mathbb{P}}_{k, n-k}$. Perciò se chiamiamo $\overline{\mathcal{W}}$ l'ideale $\left(y_{k_{+1}}, y_{k_{+2}}, \ldots, y_{n}\right)$ di $\overline{\mathbb{D}}_{k, n-k}$, abbiamo $\left(\overline{\mathfrak{a}}_{\lambda}, \overline{\mathbf{W}}\right) \neq \overline{\mathbb{D}}_{k, n-k}$. Siccome per un intero $\boldsymbol{m}$, fissato, vale $\overline{\mathfrak{q}}_{\lambda}{ }^{m} \subset \overline{\mathfrak{a}}_{\text {, }}$ (cfr. 12.4. (c)), otteniamo $\left(\overline{\mathfrak{q}}_{\gamma^{m}}{ }^{2}, \overline{\mathfrak{w}}\right) \neq \overline{\mathbb{D}}_{k, n-k}$ e perciò finalmente $\left(\overline{\mathfrak{q}}_{\lambda,}, \overline{\mathbf{w}}\right) \neq \overline{\mathbb{p}}_{k, n-k}$.

(1) e (2) mostrano, che l'ideale $\overrightarrow{\mathfrak{q}}_{\text {. }}$ soddisfa alle ipotesi valide per $\overrightarrow{\mathfrak{q}}$ nel teorema 23. Da questo teorema consegue la parte $(b)$.

(c) Dalla 14(13) segue per l'estensione in $\mathbb{P}_{n}$ ehe $\mathfrak{p} \subset \mathfrak{q}_{\lambda}$. Dunque $\mathfrak{q}_{\lambda}$ è un punto della varietà algebroide $M(\mathfrak{p})$. Ora sia $\mathfrak{g}$ un punto di $M(\mathfrak{p})$. Il nostro scopo è di dimostrare che " $g$ è un punto di una delle varietà algebroidi irriducibili $M\left(\mathfrak{q}_{2}\right)$ per $\lambda=\mu_{1}, \mu_{2}, \ldots, \mu_{h}$. Da $\mathfrak{p} \subset \mathfrak{g}$ segue per intersezione con $\overline{\mathbb{D}}_{k, n-k}$ :

$$
\overline{\mathfrak{p}}=\mathfrak{p} \cap \overline{\mathbb{p}}_{k, n-k} \subset \overline{\mathfrak{g}}=\mathfrak{g} \cap \overline{\mathbb{D}}_{k, n-k} .
$$

Per le ipotesi fatte su $\mathfrak{p}^{*}$ (cfr. 14.3.) ed essendo $\mathfrak{p}^{*} \subset \overline{\mathfrak{p}}$ si ha in conseguenza del teorema 14 , che $\mathfrak{g}$ ha una base in $\overline{\mathbb{P}}_{k, n-k}$; l'estensione di $\overline{\mathfrak{g}}$ in $\mathbb{P}_{n}$ coincide quindi con $\mathfrak{g}$ :

$$
\mathfrak{g}=\mathbb{P}_{n} \cdot \ddot{\mathfrak{g}}
$$

Sia $\mathfrak{a}^{*}$ l'ideale di annullamento di $\Phi\left(\mathfrak{p}^{*}\right)$ nell'anello $\mathbb{D}_{n}{ }^{*}$, e sia $\overline{\mathfrak{a}}=\overline{\mathbb{D}}_{k, n-k} \cdot \mathfrak{a}^{*}$ la sua estensione in $\overline{\mathbb{p}}_{k, n-k}$. Da $\mathfrak{a}^{*} \subset \mathfrak{p}^{*}$ e $\overline{\mathfrak{p}} \subset \overline{\mathfrak{g}}$ segue $\overline{\mathfrak{a}} \subset \overline{\mathfrak{g}}$. Sostituiamo ora nel prodotto $14(10)$ a $z$ la somma $u_{k+1} y_{k+1}+u_{k+2} y_{k+2}+\ldots+u_{n} y_{n}$, e poi applichiamo all'ideale primo $\overline{\mathfrak{g}}$ ed al prodotto trasformato il seguente noto teorema di algebra, cfr. [12], pag. 8 :

Siano $P\left(v_{1}, v_{2}, \ldots, v_{r}\right)$ e $Q\left(v_{1}, v_{2}, \ldots, v_{r}\right)$ due polinomi nelle indeterminate $v_{1}, v_{2}, \ldots, v_{r}$ con coefficienti in un dominio d'integrità $R$. Se tutti i coefficienti del prodotto $P \cdot Q$ appartengono ad un ideale primo di $R$, allora tutti i coefficienti di $P$ o di $Q$ devono essere contenuti in questo ideale primo.

Questo teorema dimostra che $\overline{\mathcal{g}}$ contiene almeno uno degli ideali di annullamento dei fattori in $14(10)$. Sia $\Psi_{k,}$ uno di quei fattori, cioè, per 
l'ideale di annullamento $\overrightarrow{\mathfrak{a}}_{\mathfrak{k}}$ di $\Psi_{4,}$, si abbia:

$$
\overline{\mathfrak{a}}_{\mu} \subset \overline{\mathfrak{g}} .
$$

In forza della $12(11)$ per una potenza fissata di $\overline{\mathfrak{q}}_{\mu}$ vale la relazione: $\overline{\mathfrak{q}}_{\mu}^{\sigma} \subset \overline{\mathfrak{a}}_{\mu}$ e per la (4) anche $\overline{\mathfrak{q}}_{\mu}^{\sigma} \subset \overline{\mathfrak{g}}$. Siccome $\overline{\mathfrak{g}} \dot{e}$ ideale primo, ne consegue:

$$
\overline{\mathfrak{q}}_{\mu} \subset \overline{\mathfrak{g}} .
$$

Vogliamo ora dimostrare, che $\mu$ è uno degli indici $\mu_{1}, \mu_{2}, \ldots, \mu_{h}$. A tale scopo mostreremo, che sostituendo in $\Psi_{\mu} 14(11), \Psi_{\mu}$ si muta in una potenza di $z$.

L'ideale $\overline{\mathfrak{g}}$ si annulla per $y_{1}=0, y_{2}=0, \ldots, y_{n}=0$, e, per la $(\mathbf{5}) \overline{\mathfrak{q}}_{\mu}$ gode della medesima proprieta. Introducendo in $\Psi_{\mu}$ i valori $14(11)$ si ottiene per 14.4 un prodotto $\Psi_{\mu^{0}}$ di potenze di fattori lineari $14(9)$, fra $i$ quali è anche $z$, per quanto or ora dimostrato:

(6) $\Psi_{\mu}{ }^{0}=\Psi_{\mu}\left(z \mid u_{k+1}, \ldots, u_{n},(0)_{k}\right)=z^{\alpha_{0}} \prod_{r=1}^{s}\left(z-u_{k_{+1}} y_{k+1}^{\left(\lambda_{r}\right)}-u_{k+2} y_{k+2}^{\left(\lambda_{r}\right)}-\ldots-u_{n} y_{n}^{\left(\lambda_{r}\right)}\right)^{\alpha_{r}}$

Si ha $\alpha_{a} \geq 1$. Lobbiamo dimostrare che il secondo membro della (6) è una potenza di z. La dimostrazione verrà data per assurdo, supponendo $\sum_{r=1}^{s} \alpha_{r} \geq 1$. Senza limitare la generalita del teorema inoltre possiamo supporre che esista un indice $j$, tale che $y_{k+j}^{\left(\lambda_{r}\right)} \neq 0$ per ogni $r=1,2, \ldots, s$. Infatti, essendo i punti $\boldsymbol{P}_{\lambda_{r}}=\left\{(0)_{k}, y_{k+1}^{\left(\lambda_{r}\right)}, y_{k+2}^{\left(\lambda_{r}\right)}, \ldots, y_{n}^{\left(\lambda_{r}\right)}\right\}$ tutti diversi dal punto 0 , si può trovare una -trasformazione delle coordinate $y_{k+1}, y_{k+2}, \ldots, y_{n}$, tale che p. es. le $k+j$-coordinate di essi siano diversi da 0 . Si osservi che una $\mathbb{P}$-trasforma. zione delle $n-k$ ultime coordinate non altera i risultati (a), (b), (c) del nostro teorema. In forza della 12.4 (b), l'ideale di annullamento $\overline{\mathfrak{a}}_{\mu}$ di $\Psi_{\mu}$ contiene un polinomio $\bar{q}_{k+j}$ di $y_{k+j}$ con coefficienti in $\mathbb{D}_{k}$ e con coefficiente del termine di grado massimo nguale ad 1. Questo polinomio $\vec{q}_{k+j}$ eे una potenza di un polinomio irriducibile $\bar{p}_{k_{+j}}$ dell'anello $\mathbb{P}_{k}\left[y_{k+j}\right]$, cfr. 12.4. (b). Per $y_{1}=0, y_{2}=0$, $\ldots, y_{k}=0$ il polinomio $\bar{q}_{k+j}$ si muta in

$$
\bar{q}_{k+j}\left(y_{k+j} \mid(0)_{k}\right)=y_{k+j}^{\alpha_{0}} \prod_{r=1}^{s}\left(y_{k+j}-y_{k+j}^{\left(\lambda_{r}\right)}\right)^{\alpha_{r}}, \text { efr. }(6)
$$

Siccome $\bar{q}_{k+j}$ è una potenza di $\bar{p}_{k+j}$, abbiamo per la medesima sostitu. zione :

$$
\bar{p}_{k+j}\left(y_{k+j} \mid(0)_{k}\right)=y_{k+j}^{\beta_{0}} \prod_{r=1}^{:}\left(y_{k+j}-y_{k+j}^{\left(\lambda_{r}\right)}\right)^{\beta_{r}}
$$


$\operatorname{con} \beta_{0} \geq 1$ e $\sum_{r=1}^{s} \beta_{r} \geq 1$. Applichiamo ora il lemma di HENsEL (teorema 10) al polinomio $\bar{p}_{k+j}\left(y_{k+j} \mid(y)_{k}\right)$. Questo polinomio, posto $g_{0}=y_{k+j}^{\beta_{0}}$ e $h_{0}=\prod_{r=1}^{s}\left(y_{k+j}-y_{k+j}^{\left(\lambda_{r}\right)}\right)^{\beta_{r}}$, soddisfa alle ipotesi del lemma di Henser. Infatti $g_{0}$ e $h_{0}$ non hanno un divisore comune essendo $y_{k+j}^{\left(\lambda_{r}\right)} \neq 0$, per ogni $r=1,2, \ldots, s$. Perciò $\bar{p}_{k+j}$ è prodotto dei polinomi $g\left(y_{k+j} \mid(y)_{k}\right)$ e $h\left(y_{k+j} \mid(y)_{k}\right)$ di $\mathbb{P}_{k}\left[y_{k+j}\right]$, di gradi $\beta_{0} \geqq 1$ e $\sum_{r=1}^{s} \beta_{r} \geqq 1$ rispettivamente. Questo risultato contraddice il fatto che $\bar{p}_{k+j}$ sia irriducibile in $\mathbb{P}_{k}\left[y_{k+j}\right]$. Cosi è dimostrata la nostra affermazione; cioè la funzione norma $\Psi_{\mu}$ si muta, per la sostituzione $14(11)$ in uná potenza di $z$, e quindi $\mu$ è uno degli indici $\mu_{1}, \mu_{2}, \ldots, \mu_{h}$. Per quanto asserito in (b), e già dimostrato, ed in forza delle $(3)$ e (5), consegue, mediante estensione in $\mathbb{P}_{n}$, che $\mathfrak{q}_{k} \subset \mathfrak{g}$. Allora $\mathfrak{g}$ è un punto di $M\left(\mathfrak{q}_{\mu}\right)$, per cui $\mu$ è uno degli indici $\mu_{1}, \mu_{2}, \ldots, \mu_{h}$. Ogni punto di $M(\mathfrak{p})$ appartiene quindi ad una $M\left(\mathfrak{q}_{\lambda}\right)$ per $\lambda=\mu_{1}, \mu_{2}, \ldots, \mu_{h}$. In ciò consiste appunto l'affermazione (c).

14.6. Dal teorema 39 segue immediatamente il

TEOREMA 40.

La dimensione di ogni falda di una varietà algebrica irriducibile coincide con la dimensione della varietà stessa.

14.7, Come punto generale di una varietà algebrica $M^{*}\left(\mathbf{p}^{*}\right)$ si intende un punto $\xi$, che abbia la seguente proprietà: Se $f^{*}$ è un polinomio dell'anello $\mathbb{D}_{n} *$, ed è $f^{*}(\xi)=0$, allora $f^{*}$ appartiene a $\mathfrak{p}^{*}$.

Teorema 41.

Ogni falda della varietò algebrica irriducibile $M^{*}\left(\mathfrak{p}^{*}\right)$ è punto generale di $M^{*}\left(\boldsymbol{p}^{*}\right)$.

Supponiamo che $\mathfrak{p}^{*}$ sia normalizzato, secondo quanto esposto in 14.3 ed usiamo le notazioni delle 14.3. e 14.5. Sia $M(3)$ una falda di $M^{*}\left(\mathbf{p}^{*}\right)$. Senza venir meno alla generalità possiamo supporre che $M(\xi)$ sia una falda passante per il punto 0 , (cfr. 16.1.). Colle notazioni di 14.5., sia $3=\mathfrak{q}_{\lambda}$.

Sia $f^{*}$ un polinomio dell' anello $\mathbb{P}_{n}{ }^{*}$, che si annulla su $M(3)$. Secondo la definizione 10.5 , ciò equivale alla relazione:

$$
f^{*} \in \mathfrak{q}
$$

Da essa segue:

$$
f^{*} \in \mathbf{q}_{>} \cap \overline{\mathbb{P}}_{k, n-k}=\overline{\mathbf{q}}_{\lambda} .
$$


Per dimostrare che $f^{*} \in \mathfrak{p}^{*}$, procediamo per assurdo. Dalla normalizzazione di $\mathfrak{p}^{*}$ consegue che $f^{*}$ è algebrico intero sopra $\mathbb{D}_{n}^{*} / \mathfrak{p}^{*}$ e quindi esiste nn polinomio irriducibile di $f^{*}$ :

$$
F\left(f^{*}\right)=f^{* m}+c_{1} f^{* m-1}+\ldots+c_{m-1} f^{*}+c_{m},
$$

con $c_{\lambda} \in \mathbb{P}_{k}^{*}$ per $\lambda=1,2, \ldots, m$, il quale appartiene a $p^{*}$. Per l'ipotesi della nostra dimostrazione per assurdo, abbiamo $c_{m} \neq 0$. Ora essendo $\mathfrak{p}^{*} \subset \overline{\mathfrak{q}}_{\lambda}$ (cfr. 14.13.) $\Theta \mathbb{D}_{k}^{*} \subset \mathbb{P}_{k}$, la relazione $F\left(f^{*}\right) \in \mathfrak{p}^{*}$ può essere considerata come una equazione $F\left(f^{*}\right) \in \overline{\mathbf{q}}_{\lambda}$. Se passiamo in questa equazione alle classi resto $\bmod \overline{\mathbf{q}}_{\lambda}$, per la (2) otteniamo $c_{m} \in \overline{\mathfrak{q}}_{\lambda}$. Questa relizione è incompatibile colle proprieta di $\vec{q}_{2}$, contenuta nei teoremi 39 e 40 .

\section{CAPITOLO II.}

\section{SERIE DI POTENZE CONVERGENTI}

\section{§ 15. - L' anello delle serie di potenze convergenti.}

15.1. Il seguente passaggio ad anelli di serie di potenze convergenti vuole chiarire quali nuovi aspetti acquista la nostra teoria, finora algebrica formale, in forza dell' ipotesi della convergenza. In questo capitolo l'anello dei coefficienti $\mathbb{D}$ sarà il corpo dei numeri complessi. Tra le proprietà di questo corpo, quelle aalle quali dipende l'estensione della teoria finora svolta sono le seguenti :

\section{A. Proprietà algebriche.}

1.) Il corpo dei numeri complessi ha caratteristica nulla.

2.) Il corpo dei numeri complessi è algebricamente chiuso.

\section{B. Proprietà topologiche.} proprietà.

1.) I numeri complessi posseggono un valore assoluto con le note

2.) L'insieme dei numeri complessi è completo, nel senso che vale il criterio di convergenza di CAUCHY.

15.2. Non è nostro compito sviluppare qui la teoria della convergenza delle serie infinite; tale compito spetta all'analisi. Noi ntilizziamo la seguente definizione di serie di potenze convergente: La serie di potenze

$$
f=\sum_{i_{1}=0}^{\infty} \sum_{i_{z}=0}^{\infty} \ldots \sum_{i_{n}=0}^{\infty} a_{i_{1} i_{2} \ldots i_{n}} x_{2}^{i_{1}} x_{2} i_{2} \ldots x_{n}^{i_{n}},
$$


con coefficienti complessi, si dice convergente, se esistono $n+1$ numeri $\sigma_{1}>0, \sigma_{2}>0, \ldots, \sigma_{n}>0$ e $C>0$ tali che si abbia

$$
\left|a_{i_{1} i_{2} \ldots i_{n}}\right| \leq C / \sigma_{1} i_{1} \sigma_{2}{ }^{i_{0}} \ldots \sigma_{n}^{i},
$$

per tutti $i$ valori delle $i_{1}, i_{2}, \ldots, i_{n}$ da 0 a $\infty$.

15.3. Se si tenta di applicare i risultati della teoria formale alla teoria delle serie convergenti di potenze, si incontra subito la difficolta che il teo* rema fondamentale della prima teoria, il teorema 1 , non vale più nella seconda. Le dimostrazioni dei teoremi principali della teoria formale, ciò̀ dei teoremi $2,3,4,6,7,10,11$, devono percio essere rivedute. Come risulta palese dalla definizione di convergenza qui adottata, il metodo più opportuno nelle dimostrazioni è quello della maggiorazione. La sua efficacia proviene dalla semplice proprietà che dall'assoluta convergenza di una serie infinita di numeri complessi segue la sua convergenza. Con $i$ metodi noti della teoria delle serie infinite si dimostra che somme e prodotti di serie infinite convergenti sono convergenti. $\mathrm{Ne}$ segue :

Teorema 42.

Le serie convergenti di potenze dell'anello $\mathbb{D}_{n}$ formano un anello.

15.4. Col metodo della maggiorazione si prova anche il seguente teorema, che costituisce il termine di passaggio all' ordine d'idee analitico.

Teorema 43.

Sia $f$ una serie di potenze convergente dell'anello $\mathbb{D}_{n}$. Valgano le $15(1)$ e la 15(2). Sia $\left\{x_{1}{ }^{0}, x_{2}{ }^{0}, \ldots, x_{n}{ }^{0}\right\}$ un sistema di numeri complessi per il quale si $a b b i a$

$$
\left|x_{i}{ }^{n}\right|<\sigma_{i} \quad(i=1,2, \ldots, n) .
$$

Allora la serie infinita

$$
f_{\mathrm{v}}=\sum_{i_{1}=0}^{\infty} \sum_{i_{2}=0}^{\infty} \ldots \sum_{i_{n}=0}^{\infty} a_{i_{1} i_{2} \ldots i_{n}}\left(x_{1}^{0}\right)^{i_{1}}\left(x_{2}\right)^{i_{2}} \ldots\left(x_{n}^{0}\right)^{i_{n}}
$$

risulta convergente.

La possibilita di sostituire sistemi di valori nelle serie di potenze è la più importante estensione del metodo finora puramente formale. Si ricordi che nella teoria formale il solo sistema che si poteva sostituire era il sistema nullo. Ora invece si possono ricercare l'insieme degli zeri di una serie di potenze; questo nuovo ordine di questioni richiede un nuovo tipo di terminologia e di notazioni, e precisamente quello della topologia e della teoria delle funzioni. 
Consideriamo ora le indeterminate $x_{1}, x_{2}, \ldots, x_{n}$ come variabili e parliamo del punto di coordinate $x_{1}, x_{2}, \ldots, x_{n}$ nello spazio complesso $C_{n}$ di dimen. sione $n$. Dopo di ohe possiamo utilizzare tutti i concetti della topologia.

15.5. Mediante concetti topologici possiamo precisare la definizione 15.2.; premettiamo talune notazioni :

Il campo cilindrico

$$
\left|x_{i}\right|<\rho_{i}, \rho_{i}>0, \quad(i=1,2, \ldots . n)
$$

sarà denotato con $\mathfrak{3}(\rho)=\mathfrak{3}(\rho)_{n}=\mathfrak{3}\left(\rho_{1}, \rho_{2}, \ldots, \rho_{n}\right) ;$ con $\mathfrak{3}(\rho)_{n}$ sarà indicato il suo involucro chiuso

$$
\left|x_{i}\right| \leq p_{i} \quad(i=1,2, \ldots, n) .
$$

La serie di potenze $f$ data dalla $15(1)$ si dice convergente nel campo cilindrico $3(\rho)_{n}$ se per ogni $0<\sigma_{i}<\rho_{i}, i=1,2, \ldots, n$, valgono limitazioni del tipo della 14(2), nella quale $C$ puó dipendere dalle $\sigma_{1}, \sigma_{2}, \ldots, \sigma_{n}$. In questo caso chiamiamo $3(\rho)_{n}$ un cilindro di convergenza di $f$.

Sía $f$ una serie di potenze convergente. $f$ possiede un campo di conver. genza (assoluta), ofr. [15], pag. 38. L'intorno di questo campo sia indicato con $\mathbf{R}(f)$. ì chiaro che $\mathbb{R}(f)$ contiene un cilindro di convergenza $\mathfrak{3}(p)_{\boldsymbol{n}}$. In $\mathbf{R}(f)$ si può concepire $f$ come funzione delle $n$ variabili complesse $x_{2}, x_{2}, \ldots$, $x_{n}$, definita nel modo seguente:

Se $x^{0}=\left\{x_{1}{ }^{0}, x_{i}{ }^{0}, \ldots, x_{n}{ }^{0}\right\}$ è un punto di $\mathbb{R}(f)$, allora il valore della funzione $f$ nel punto $x^{0}$ sia il numero complesso $f_{0}$ in 15.4. La funzione $f$ si dice olomorfa in $\mathbf{R}(f)$. Poichè essa definita per ora solo nell'intorno $\mathbb{R}(f)$ del suo campo di convergenza, si pone la questione di estendere eventualmente la sua definizione a campi più ampi. Questo problema è connesso con quello del prolungamento analitico, ma fa parte di un ordine di problemi di cai qui non ei occupiamo, in quanto noi puntiamo esclnsivamente a risultati validi in un intorno - del resto comunque piccolo - di un punto di $C_{n}$. Limitiamo cioè la nostra ricerca ai cosiddetti problemi locali. Si presenta qui di estremo interesse l'insieme degli zeri delle serie di potenze o degli ideali di serie di potenze. Usiamo per tali insiemi il termine « varietà analitiche» $\theta$ accentuiamo cosi la differenza tra queste e le varietà algebroidi del $\S 10$. Gli ultimi concetti considerati richiedono tuttavia talune precisazioni, per le quali è necessario studiare più accuratamente lo serie di potenze nei punti del loro campo di convergenza. 


\subsection{TEOREMA 44.}

Sia $f$ una serie di potenze convergente dell'anello $\mathbb{D}_{n}$, avente il cilindro di convergenza $3(\rho)$. Sia $x^{0}$ un punto di $3(\rho)$. Poniamo

$$
x_{i}^{\prime}=x_{i}-x_{i}^{0} \quad(i=1,2, \ldots, n)
$$

si ottiene così una serie di potenze convergente $f^{\prime}$ dell'anello $\mathbb{P}_{n}^{\prime}=\mathbb{P}\left[\left[x_{1}^{\prime}, x_{2}^{\prime}, \ldots, x_{n}^{\prime}\right]\right]$, con un cilindro di convergenza $\mathfrak{3}^{\prime}\left(\rho^{\prime}\right)$ e che gode della proprietà seguente: se $\bar{x}$ è un punto di $3(\rho)$ e se insieme con le

$$
\bar{x}_{i}^{\prime}=\bar{x}_{i}-x_{i}{ }^{0}
$$$$
(i=1,2, \ldots, n)
$$

si ha $\bar{x}^{\prime} \in \mathfrak{Z}^{\prime}\left(\rho^{\prime}\right)$, allora vale la relazione.

$$
f(\bar{x})=f^{\prime}\left(\bar{x}^{\prime}\right) .
$$

Chiamiamo $f^{\prime}$ il prolungamento analitico di $f$ nel punto $x^{0}$.

La dimostrazione si ottiene sostituendo $x_{i}=x_{i}^{\prime}+x_{i}{ }^{0}$ nella $f(i=1,2, \ldots, n)$ e ordinando poi $f$ secondo le potenze delle $x_{i}^{\prime}$. Tale ordinamento e consentito dall' assoluta convergenze della serie (cfr. [15], pag. 51).

Il concetto di prolungamento analitico si pnò estendere agli ideali di serie di potenze.

Sia a un ideale di serie convergenti di potenze dell'anello $\mathbb{D}_{n}$. Per a esista una base $f_{1}, f_{2}, \ldots, f_{n}$, i cui elementi siano convergenti in $3(\rho)$. Sia $x^{0}$ un punto di $3(\rho)$ e $f_{1}^{\prime}, f_{2}^{\prime} \ldots, f_{n}{ }^{\prime}$ siano i prolungamenti analitici di $f_{1}, f_{2}, \ldots$, $f_{\prime \prime}$ in $x^{0}$, L'ideale $\mathfrak{a}^{\prime}$ generato nell' anello di serie di potenze $\mathbb{P}_{n}^{\prime}$ dagli elementi $f_{1}^{\prime}, f_{2}^{\prime}, \ldots, f_{n}^{\prime}$ si dice prolungamento analitico dell'ideale a nel punto $x^{0}$.

15.7. Applicando il teorema 44 si possono formulare con maggior generalità taluni risultati validi per le serie formali di potenze. Questa possibilità dipende dal fatto che le serie convergenti di potenze ammettono un maggior numero di sostituzioni che le serie formali.

Per trasformazione analitica intendiamo una sostituzione $(x)_{n}=T(y)_{m}$ data dalle formule

$$
x_{\nu}=g_{v}\left(y_{1}, y_{2}, \ldots, y_{m}\right) \quad(v=1,2, . ., n),
$$

nelle quali. le $g_{v}$ sono serie di potenze convergenti.

Teorema 45.

Sia $f$ una serie di potenze convergente dell'anello $\mathbb{D}_{n}$. Sia $(x)_{n}=T(y)_{m}$ una trasformazione analitica di equazioni $15(10)$, tale che il punto yo appar. tenga all'intorno del campo di convergenza di tutte le serie $g_{1}, g_{2}, \ldots, g_{n} e$ 
che $x^{0}=T\left(y^{0}\right)$ appartenga all' intorno del campo di convergenza di $f$. Allora $f$ viene trasformata dalla $T$ in una serie di potenze convergente dell'anello $\mathbb{P}\left[\left[y_{1}-y_{1}^{0}, y_{2}-y_{2}^{0}, \ldots, y_{m}-y_{m}^{0}\right]\right]$.

La dimostrazione segue dal teorema di CADCHY sull'ordinamento, che è applicabile alle serie assolutamente convergenti (cfr. [15], pag. 51).

15.8. Il teorema sulle funzioni implicite (Teorema 3) si può enunciare ora al modo seguente:

TEOREMa 46.

Nell'anello $\mathbb{P}_{1+m}$ siano date le serie di potenze convergenti $F_{i}\left(x_{1}, x_{2}, \ldots\right.$, $\left.x_{n}, y_{1}, y_{2}, \ldots, y_{m}\right)(i=1,2, \ldots, m)$, che si annullino nel punto $\left\{x^{0}, y^{0}\right\}, i l$ quale appartenga all'intorno del campo di convergenza di tutte le serie $F_{i}, i=1,2, \ldots, n$. Il determinante funzionale

$$
\frac{\partial\left(F_{1}, F_{2}, \ldots, F_{m}\right)}{\partial\left(y_{1}, y_{2}, \ldots, y_{m}\right)}
$$

sia diverso da zero in tale punto. Allora esistono $m$ serie di potenze convergenti $g_{1}, g_{2}, \ldots, g_{m}$ dell'anello $\mathbb{D}\left[\left[x_{1}-x_{1}{ }^{0}, x_{2}-x_{2}{ }^{0}, \ldots, x_{n}-x_{n^{0}}{ }^{0}\right]\right]$, tale che per $i$ prolungamenti analitici $F_{i}^{\prime}$ delle $F_{i}$ nel punto $\left\{x^{0}, y^{0}\right\}$ si abbia

$$
F_{i}^{\prime}\left(x_{1}-x_{1}{ }^{0}, x_{2}-x_{2}{ }^{0}, \ldots, x_{n}-x_{n}{ }^{0}, g_{1}, g_{2}, \ldots, g_{m}\right)=0 \quad(i=1,2, \ldots, m)
$$

Analogamente si generalizzano i teoremi 4 e 5 .

15.9. Il trasporto del teorema della base di RückERT agli ideali di serie convergenti di potenze richiede un procedimento diverso da quello del $\$ 4$; infatti la dimostrazione di 4.1. non è più valida. Secondo il procedimento di RuOKERT ai dimostra prima la validità della formula di WEIERstrass e del teorema di preparazione di WrIERSTRASS nell'anello delle serie convergenti di potenze.

Thorema 47.

Se si applicano a serie convergenti di potenze le regole di calcolo che intervengono nell'applicazione della formula di Weierstrass ovvero del teorema di preparazione di Weierstrass, si ottengono serie convergenti di potenze.

Per le semplici dimostrazioni della convergenza rinviamo a [1]. pag. 186.

15.10. Da questo teorema consegue il lemma di HENSEL per polinomi con coefficienti convergenti nel modo seguente: 
Usiamo le notazioni del $\S 5$, ma ora $F$ sia un polinomio con coefficienti convergenti. Basta trattare il caso $g_{0}(y)=\left(y-y_{0}\right)^{p}$. Dal lemma di HenseL consegue una rappresentazione univoca:

$$
F\left(y \mid(x)_{k}\right)=G\left(y \mid(x)_{k}\right) \cdot H\left(y \mid(x)_{k}\right),
$$

della quale finora sappiamo soltanto che i coefficienti di $G$ e $H$ sono serie di potenze formali. Tuttavia, sostituendo $x_{1}=0, x_{2}=0, \ldots, x_{n}=0$ in $G$, si ottiene $\left(y-y_{0}\right)^{p}$ ed $H$ non si annulla per $x_{1}=0, x_{2}=0, \ldots, x_{k}=0, y=y_{0}$. La rappresentazione (1) coincide esattamente con quella che dà il teorema di WEIERSTRAss, applicato ad $F$ nel punto $x_{1}=0, x_{2}=0, \ldots, x_{k}=0, y=y_{0}$. Per il teorema $47, G$ e $H$ devono essere delle serie di potenze convergenti in $x_{1}, x_{2}, \ldots, x_{k}, y-y_{0}$. Perciò i coefficienti di $G$ e $H$ in (1) sono convergenti.

15.11. Dopo che ei siamo assicurati della ralidita dei teoremi di Wererstrass nel campo delle serie convergenti di potenze, si può dimostrare il teorema della base per ideali di serie convergenti di potenze, seguendo lo stesso metodo che si utilizza per gli ideali di polinomi; efr. [1], pag. 204 ovvero [5], pag. 142 .

\section{Teorema 48.}

$L$ 'anello delle serie convergenti di potenze è noetheriano.

Conduciamo la dimostrazione per induzione completa rispetto ad $n$, numero delle variabili. Per $n=0$ il teorema è vero, perchè $\mathfrak{P}$, essendo un corpo, è noetheriano. Si supponga il teorema dimostrato per gli ideali dell'anello delle serie convergenti di potenze in $n-1$ variabili. Sia $a$ un ideale di $\mathbb{P}_{n} \theta p$ un elemento di a. Mediante la trasformazione di coordinate $y=L(x)$ sia $p^{*}=p\left(L^{-1}(y)\right)$ una serie privilegiata rispetto a $y_{n}$. L'ideale a sia trasformato dalla $L$ nell'ideale $a^{*}$ di $\boldsymbol{D}_{n}{ }^{*} y$. Basta provare che $a^{*}$ e noetheriano. Per la formula di Wrierstrass ogni elemento $f^{*}$ di $\mathbb{P}_{n} *$ ammette una rappresentazione del tipo $f^{*}=h^{*} p^{*}+\bar{f}$ con $h^{*} \in \mathbb{D}_{n}{ }^{*}$ e $\bar{f} \in \mathbb{D}_{n-1}^{*}\left[y_{n}\right]$. Inoltre il grado del polinomio $f$ in $y_{n}$ è minore di $r$, se mediante la sostitu. zione $y_{1}=0, y_{2}=0 \ldots, y_{n-1}=0$ la serie $p^{*}$ si riduce a $\alpha \cdot y_{n}{ }^{*}(\alpha \neq 0)$. Se applichiamo tale formula a tutti gli elementi di a, i coefficienti di $y_{n}{ }^{r-1}$ nei polinomi formano un ideale in $\mathbb{D}_{n-1}^{*}$. Per l'ipotesi di induzione questo ideale ha una base finita $\gamma_{1}, \gamma_{2}, \ldots, \gamma_{6}$. Per ogni $\gamma_{i}$ scegliamo in $a^{*}$ un polinomio $\bar{g}_{1}$ contenente l'addendo $\gamma_{i} y_{n}{ }^{r-1}$. Ogni elemento di $a^{*}$ è rappresentabile come somma di un elemento dell' ideale $a_{1}^{*}=\left(p^{*}, \bar{g}_{1}, \bar{g}_{2}, \ldots, \bar{g}_{s}\right)$ e di un polinomio in $y_{n}$ di grado minore di $r-1$. Ammettiamo ora di aver scelto gli elementi $p^{*}, \bar{g}_{1}, \bar{g}_{2}, \ldots, \bar{g}_{t}$ nell'ideale $a^{*}$ in modo tale che ogni elemento $f^{*}$ di $\mathfrak{a}^{*}$ sia somma di un elemento dell'ideale $a_{i}^{*}=\left(p^{*}, \bar{g}_{1}, \bar{g}_{2}, \ldots, \bar{g}_{t}\right)$ e di un polinomio in $y_{n}$ di grado minore di $r-i$. Se $t^{*}$ desorive l'ideale $a^{*}$, i coefficienti di $y_{n}{ }^{r-1-1}$ 
in tali polinomi descrivono un ideale in $\mathbb{D}_{n-1}^{*}$. Per l'ipotesi di induzione tale ideale ammette una base finita $\gamma_{t+1}, \gamma_{t+2}, \ldots, \gamma_{u}$; sia allora $\bar{g}_{t+j}$ un polinomio in $\mathfrak{a}^{*}$ che contiene l'addendo $\gamma_{t+j} y_{n}{ }^{r-i-1}$. Ogni elemento di $\mathfrak{a}^{*}$ è somma di un elemento dell'ideale $\mathfrak{a}_{i+1}^{*}=\left(p^{*}, \bar{g}_{1}, \bar{g}_{2}, \ldots, \bar{g}_{u}\right)$ e di un polinomio in $y_{n}$ di grado minore di $r-i-1$. Poichè per $i=r-1$ si ha $\mathfrak{a}_{r-1}^{*}=\mathfrak{a}^{*}$, abbiamo costruito una base finita per $\mathfrak{a}^{*}$.

15.12. I risnltati di questo paragrafo si possono cosi riassumere: Tutte le più importanti operazioni di calcolo della teoria degli anelli di serie di potenze non fanno uscire dall'anello delle serie convergenti di potenze. Gli ulteriori teoremi sulle serie formali di potenze contenuti nei paragrafi 6-12 sono orá conseguenza di questi risultati ; essi valgono dunque anche nell'ipotesi che tutte le serie di potenze che intervengono siano convergenti. Se dunque tutta la teoria degli ideali di serie formali di potenze si può trasferire al caso ora trattato, ̀̀ tuttavia da tener presente che le ipotesi più strette che ora si ammettono portano a nuovi enunciati; ed è facile immagi. nare in quale direzione siano da ricercarsi tali nuove proprietà : saranno quelle che si riferiscono alle proprietà topologiche degli insiemi di zeri degli ideali di serie di potenze.

\section{§ 16. - Varietà analitiche.}

16.1. Il concetto più adatto allo studio degli ideali di serie di potenze convergenti mediante metodi geometrici è quello di germe di insiemi.

Sia $R$ uno spazio topologico e $P$ un punto di $R$. Gli insiemi di punti, $\boldsymbol{\omega}_{1}$ ed $\boldsymbol{\omega}_{2}$, di $R$, si dicono equivalenti in relazione a $P$ quando esiste un intorno $U(P)$ di $P$, tale che si abbia

$$
\boldsymbol{\Omega}_{1} \frown U(\boldsymbol{P})=\boldsymbol{M}_{2} \frown U(\boldsymbol{P})
$$

Le olassi determinate da questa relazione di equivalenza si dicono germi di insiemi.

Si definiscono poi, in maniera univoca, le unioni e le intersezioni di germi, come le classi determinate dall' unione e dall'intersezione di insiemi rappresentanti i germi considerati.

16.2. A noi interessa una particolare classe di germi di insiemi. Perverremo ad essa dopo aver definito il coneetto di varieta analitica.

Sia $C_{n}$ lo spazio complesso di dimensione $n$. Sia $P_{0}$ un punto di $C_{n}$, di coordinate $\left\{x_{1}{ }^{0}, x_{2}{ }^{0}, \ldots, x_{n}{ }^{0}\right\}$. Con $p_{11}\left(P_{0}\right)$ indicheremo l'anello delle serie convergenti di potenze di $x_{1}-x_{1}{ }^{0}, x_{2}-x_{2}{ }^{0}, \ldots, x_{n}-x_{n}{ }^{0} ;$ siano $f_{1}, f_{2}, \ldots, f_{\text {. fun }}$ zioni di $D_{n}\left(P_{0}\right)$; sia $U\left(P_{0}\right)$ un intorno di $P_{0}$, il quale giaccia nel campo di 
convergenza di tutte le serie $f_{\nu}$. Si chiama varietà analitica in $U\left(P_{0}\right)$ l'insieme degli zeri comuni di $f_{1}, f_{2}, \ldots, f_{r}$, i quali stanno in $U\left(P_{0}\right)$.

Un germe di insiemi di $C_{n}$, in un punto $P_{0}$, si dice analitico, se ammette come rappresentante una varietà analitica.

\subsection{TEOREMA 49 .}

Ogni ideale a dell'anello di serie di potenze $\mathbb{P}_{n}\left(P_{0}\right)$ determina univocamente un germe analitico $\mathbb{\Pi D ( a )}$ di $P_{0}$ come germe di insiemi degli zeri di a.

Se $f_{1}, f_{2}, \ldots, f_{r}$ è una base di a, esiste un intorno $U\left(P_{0}\right)$ di $P_{0}$, nel quale tutte le serie $f_{v}$ convergono. 1 loro zeri comuni riempiono in $U\left(P_{0}\right)$ una varietà analitica $A(\mathfrak{a})$, la quale definisce in $P_{0}$ un germe di insiemi $\Omega(\mathbb{D}(\mathfrak{a})$. Per provare che $\Omega(\mathfrak{D}(\mathfrak{a})$ è univocamente determinato da $\mathfrak{a}$, occorre dimostrare che $\Omega(\mathfrak{a})$ e indipendente dalla base di a. Sia infatti $g_{1}, g_{2}, \ldots, g_{s}$ un'altra base di a, e tali serie siano convergenti nell' intorno $V P_{0}$ ) di $P$, i loro zeri comuni in $V\left(P_{0}\right)$ costituiscono allora una varietá analitica $B(\mathfrak{a})$. Ci basta ormai dimostrare che esiste un intorno $W\left(P_{0}\right)$ di $P_{0}$, tale che si abbia:

$$
A(\mathbf{a}) \frown W\left(P_{0}\right)=B(\mathbf{a}) \frown W\left(P_{0}\right)
$$

Poichè le $f_{\lambda}$ e le $g_{\mu}$ costituiscono due basi, valgono le relazioni

$$
\begin{array}{ll}
f_{\lambda}=a_{\lambda 1} g_{1}+a_{\lambda, 2} g_{2}+\ldots+a_{\lambda, g} g, & (\lambda=1,2, \ldots, r) \\
g_{\mu}=b_{\mu 1} f_{1}+b_{\mu 2} f_{2}+\ldots+b_{\mu \mu} f_{r} . & (\mu=1,2, \ldots, s),
\end{array}
$$

con $a_{\lambda, i}$ e $b_{\mu j}$ elementi di $\mathbb{P}_{n}\left(P_{0}\right)$. Sia $W\left(P_{0}\right)$ un intorno di $P_{0}$, nel quale con. vergono tutte le serie che compariscono nelle relazioni soritte. Da (2) e (3) segue allora immediatamente la (1).

Le precedenti considerazioni mostrano che in nessun caso un ideale di serie di potenze puo determinare univocamente una varieta analitica, in quanto questa dipende non solo dall'ideale, ma anche dalla scelta di una base; l'ideale determina invece il germe analitico d'insiemi. Si noti ora che le proprietà degli ideali di serie di potenze, che sono quelle che presentano il maggior interesse, si ritrovano come proprieta dei germi analitici e devono quindi caratterizzare ogni rappresentante del germe. Se dunque si cercano proprietà dei germi analitici d'insiemi, si può studiare un qualsiasi rappresentante del germe; ed esso si può scegliere in base a criteri di opportunità ; in questa scelta si è guidati dai teoremi di rappresentazione dei paragrafi 9 e 12. 
16.4. Il teorema 49 ammette il seguente teorema inverso:

Teorema 50.

Il germe analitico d'insiemi, $\Omega$, di $P_{0}$ determina univocamente un ideale $\mathfrak{i}(\mathrm{MD})$ nell'anello di serie di potenze $\mathbb{D}_{n}\left(P_{0}\right)$, cioè l'ideale di tutte le serie di $\mathbb{P}_{n}\left(P_{0}\right)$, che svaniscono su $\mathbb{I D}$.

(a) Si dice che una serie di potenze $f \in \mathbb{D}_{n}\left(P_{0}\right)$, svanisce sulla varietà analitica $A$, che è definita nell' intorno $U\left(P_{0}\right)$ di $P_{0}$, se $U\left(P_{0}\right)$ appartiene al campo di convergenza di $f$ e se $f$ si annulla in tutti i punti di $A$.

(b) Si dice che la serie di potenze $f \in \mathbb{P}_{1}\left(P_{0}\right)$ svanisce sul germe analitico d'insiemi $\Omega$ di $P_{0}$, se esiste un rappresentante di MD, snl quale $f$ svanisca secondo la definizione $(a)$.

(c) La totalità delle serie di $\mathbb{P}_{n}\left(P_{0}\right)$. che svaniscono su $\mathbb{M}$, forma un ideale in $\mathbb{P}_{n}\left(P_{0}\right)$, precisamente l'ideale $\mathfrak{i}(\Omega \mathbf{S})$.

16.5. La legge di associazione $\Omega(\mathfrak{D}(\mathfrak{a})$ e la $\mathfrak{i}(\Omega)$ soddisfanno alle seguenti relazioni :

(1) Da $\mathfrak{a} \subset \mathfrak{b}$ segue $\mathbb{M}(\mathfrak{b}) \subset \mathbb{M}(\mathfrak{a})$.

(2) Se $\mathfrak{a}=(\mathfrak{b}, \mathfrak{c})$, allora $\operatorname{MD}(\mathfrak{a})=\operatorname{MD}(\mathfrak{b}) \cap \Omega(\mathbb{D}(\mathfrak{c})$.

(3) Se $\mathfrak{a}=\mathfrak{b} \cap \mathfrak{c}$, allora $\mathbb{M D}(\mathfrak{a})=\mathbb{M D}(\mathfrak{b}) \cup \mathbb{M D}(\mathbf{c})$.

(4) Se $\mathfrak{q}$ ¿̀ un ideale primario, associato all'ideale primo $\mathfrak{g}$, allora $\boldsymbol{m i}(\mathfrak{q})=\mathbb{M}(\mathfrak{g})$.

(5) $\mathbf{m}(\mathbf{i}(\Omega \mathbf{D}))=\mathbf{m}$.

(6) Da $\Omega_{1} \subset \mathbb{D}_{2}$ segue $\mathfrak{i}\left(\Omega \mathrm{D}_{2}\right) \subset \mathfrak{i}\left(\Omega \mathrm{D}_{1}\right)$.

(7) Se $\Omega=\Omega_{1} \cup \mathbb{M}_{2}$, allora $\mathfrak{i}(\Omega \mathbb{D})=\mathfrak{i}\left(\Omega \mathbb{D}_{1}\right) \cap \mathfrak{i}\left(\Omega \mathbb{D}_{2}\right)$.

Tali relazioni si dimostrano stabilendole per opportuni rappresentanti dei germi considerati. Si consideri p. es. la (5): il germe (iD abbia un rappresentante determinato dalle equazioni $f_{i}=0(i=1,2, \ldots, r)$; allora, per 16.4, le serie $f_{1}$ appartengono a $\mathfrak{i}(\mathbb{D})$, e da (1) segue $\Omega(\mathfrak{D}(\Omega(\mathbb{D})) \subset \Omega(\mathbb{D}$. Come ideale di $D_{n}\left(P_{0}\right)$, l'ideale $\mathfrak{i}(\Omega)$ possiede una base finita $g_{j}(j=1,2, \ldots, s)$, e le serie $g_{i}$ svaniscono sopra un rappresentante $A$ di $M$. D' altronde le equazioni $g_{j}=0(j=1,2, \ldots, s)$ definiscono un rappresentante $B$ di $\Omega(\mathbb{D}(\mathbf{i}(\mathbb{M}))$. Si ha $A \subset B$ e perciò anche $\boldsymbol{S} \subset \boldsymbol{( D}(\mathbf{i}(\boldsymbol{D}))$; e da questa segue la (5).

16.6. Un germe analitico d'insiemi $\mathrm{m}$ di $P_{0}$ si dice riducibile se esistono due germi analitici d'insiemi $\boldsymbol{M}_{1}, \boldsymbol{\Omega}_{2}$, di $P_{0}$, tali che $\mathbb{M}=\boldsymbol{M}_{1} \cup \mathbb{M}_{2}$, senza che $\boldsymbol{I}_{1}$ sia parte di $\mathbf{M}_{2}$ o $\mathbf{S}_{2}$ sia parte di $\mathbf{M}_{1}$. Se non esiste un siffatto spezzamento di $\boldsymbol{\Omega}$, questo si dice irriducibile, o, brevemente, "germe primo" 
di $P_{0}$. Una varietà analitica si dice irriducibile in $P_{0}$ se è rappresentante di un germe d'insiemi analitico ed irriducibile, di $P_{0}$.

TEOREMA 51.

Un germe analitico d'insiemi M $\mathrm{ND} P_{\mathrm{u}} \grave{e}$ un germe primo se e soltanto se il suo ideale $\mathrm{i}(\mathrm{\Omega D})$ è primo.

Mostreremo che la riducibilita di $\mathfrak{i}(\mathbb{M})$ segue dalla riducibilita di $\mathbb{M}$, $\theta$ viceversa.

(a) $I l$ germe $\mathbb{M}$ sia riducibile, e sia $\mathbb{M}=\mathbb{D}_{1} \cup \mathbb{D}_{2}$. Allora per 16.5. (7): $\mathfrak{i}(\Omega \mathbb{D})=\mathfrak{i}\left(\Omega \mathbb{D}_{1}\right) \frown \mathfrak{i}\left(\Omega \mathbb{D}_{2}\right)$. Da $\mathfrak{i}\left(\Omega \mathbb{D}_{\lambda}\right) \subset \mathfrak{i}\left(\mathbb{M}_{k_{k}}\right)(\lambda, \mu=1,2)$ seguirebbe per 16.5. (1) e (5), $\Omega \mathbb{D}_{\mu} \subset \Omega \mathbb{D}_{\lambda}$, e ciò non è vero per ipotesi $(\lambda \neq \mu)$.

(b) $\mathfrak{i}(\Omega \mathrm{D})$ non sia un ideale primo, e sia $\mathfrak{i}(\Omega \mathbb{D})=\mathfrak{b} \frown \mathfrak{c}$ una scomposizione tale che $b$ non è contenuto in $c$ e $c$ non è contenuto in $\mathfrak{b}$. Per 16.5. (5) $\theta$ (3) si ha $\mathbb{M D}=\Omega \mathbb{D}(\mathfrak{b}) \cup \Omega \mathbb{D}(\mathcal{C})$. Poniamo $\mathfrak{i}(\Omega \mathbb{D}(\mathfrak{b}))=\mathfrak{b}_{1}$ e $\mathfrak{i}(\Omega \mathbb{D}(\mathcal{C}))=\mathfrak{c}_{1} ;$ si ha allora per 16.5. (7) $\mathfrak{i}(\mathbb{M})=\mathfrak{b}_{1} \frown \mathfrak{c}_{1}$. Poichè $\mathfrak{b} \subset \mathfrak{b}_{1} e \mathcal{C} \subset \mathfrak{c}_{1}$, se fosse p. es. anche

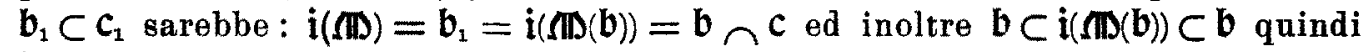
$\mathfrak{i}(\Omega \mathbb{D})=\mathfrak{b}$. Allora avremmo $\mathfrak{b} \subset \mathfrak{C}$, che non a vero. Analogamente si esclude che sia $c_{1} \subset b_{1}$. Per 16.5. (6) se ne trae che $\Omega(b)$ non sta in $\Omega(\mathcal{C})$ e che $\Omega(C)$ non sta in $M(b)$ e quindi che $\Omega(\grave{D}$ è riducibile.

16.7. Si tratta di formulare e di dimostrare il così detto secondo teorema di WEIERSTRASS sullo spezzamento di una varietà analitica in varietà irriducibili e, per quanto già sappiamo, questo è sostanzialmente un teorema sullo spezzamento di un germe analitico di insiemi (cfr. [15], p. 131).

Teorema 52.

Sia a un ideale dell'anello di serie di potenze $\mathbb{D}_{n}\left(P_{0}\right), M(\mathfrak{a})$ la sua varietà algebroide (cfr. \& 10) e $1 \mathrm{D}(\mathfrak{a})$ il suo germe analitico d'insiemi. Sia

$$
M(\mathfrak{a})=M\left(\mathfrak{g}_{1}\right) \cup M\left(\mathfrak{g}_{2}\right) \cup \cdots \cup M(\mathfrak{g},)
$$

la rappresentazione di $M(\mathfrak{a})$ come insieme unione di varietà irriducibili $\boldsymbol{M}\left(\mathfrak{g}_{i}\right)$ $(i=1,2, \ldots, r)$; inoltre $i \mathfrak{g}_{i}$ sono ideali primi dell' anello $\mathbb{P}_{n}\left(P_{0}\right)$, cioè determi. nano esattamente le componenti isolate dell'ideale a. Sotto queste ipotesi la formula

$$
\Pi \mathfrak{D}(\mathfrak{a})=\Omega \mathfrak{D}\left(\mathfrak{g}_{1}\right) \cup \Lambda \mathrm{D}\left(\mathfrak{g}_{2}\right) \cup \cdots \cup \mathfrak{M}\left(\mathfrak{g}_{r}\right)
$$

fornisce la rappresentazione del germe analitico $\mathrm{MD}(\mathfrak{a})$ come unione dei germi primi $\mathfrak{\Omega}\left(\mathfrak{g}_{i}\right)(i=1,2, \ldots, r)$; questa unione è inaccessibile, cioè nessuno dei germi primi $\Omega \mathrm{D}\left(\mathfrak{g}_{i}\right)$ è contenuto nell'unione dei rimanenti.

(a) Sia

$$
\mathfrak{a}=\mathfrak{q}_{1} \frown \mathfrak{q}_{2} \frown \cdots \cap \mathfrak{q}_{r} \cap \mathfrak{q}_{r+1} \cap \mathfrak{q}_{r+2} \cap \cdots \cap \mathfrak{q}_{r+\mathfrak{*}}
$$


una rappresentazione di a come intersezione di ideali primari. L'ideale primo associato a $\mathfrak{q}_{i}$ sia $\mathfrak{g}_{i}(i=1,2, \ldots, r+s)$. Le prime $r$ componenti sono perciò isolate, mentre le seguenti sono immerse, oioè per ogni $j=1,2, \ldots, s$ esiste un $\mathfrak{g}_{i(j)}$, con $i(j) \leq r$, tale ohe $\mathfrak{g}_{i(j)} \subset \mathfrak{g}_{r i j}$. Per 16.5. (3) e (4) abbiamo:

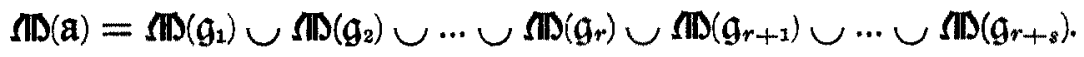

Da $\mathfrak{g}_{i(j)} \subset \mathfrak{g}_{r+j}$ segue, per la 16.5. (1), $\mathbb{M D}\left(\mathfrak{g}_{r+j}\right) \subset \Omega\left(\mathbb{D}\left(\mathfrak{g}_{i(j)}\right)\right.$ e da ciò la $16(3)$.

(b) Occorre ora dimostrare che il germe analitico dj un ideale primo è irriducibile. Per il Teorema 51, tale affermazione equivalente alla proposizione

$$
\mathfrak{i}(\mathbf{M D}(\mathfrak{g}))=\mathfrak{g} \text {, se } \mathfrak{g} \text { è un ideale primo. }
$$

La dimostrazione di quest'ultima sar data nel seguito, efr. Teorema 57.

(c) Se prendiamo per dimostrata la formula 16(4), possiamo provare facilmente l'inaccorciabilità della rappresentazione $16(3)$. All' nopo basta dimostrare quanto segue:

Sia $\mathfrak{g}$ un ideale primo e $\mathbb{M D}(\mathfrak{g})$ sia contenuto nell'unione dei germi analitici $\mathbb{S}_{1}$ ed $\mathbb{M D}_{2}$; allora $\mathbb{M}(\mathfrak{g})$ appartiene a $\mathbb{M D}_{1}$ ovvero a $\mathbb{M D}_{2}$.

Da $\mathbb{M D}(\mathfrak{g}) \subset \mathbb{D}_{1} \cup \mathbb{M}_{2}$ segue, per la 16.5 . (6) e (7) e per la formula $16(4)$, che $\mathfrak{i}\left(\mathbb{D}_{1}\right) \cap \mathfrak{i}\left(\mathbb{D}_{2}\right) \subset \mathfrak{i}(\mathbb{M}(\mathfrak{g}))=\mathfrak{g}$. Poichè $\mathfrak{g}$ è un ideale primo, si ha $\mathfrak{i}\left(\boldsymbol{M}_{1}\right) \subset \mathfrak{g}$ ovvero $\mathfrak{i}\left(\mathbb{N}_{2}\right) \subset \mathfrak{g}$. Nel primo caso dalla 16.5 . (1) e (5) segue $\mathbb{M}(\mathfrak{g}) \subset \mathbb{M}_{1}$ e nel secondo, per la stessa ragione, $\mathbb{\Lambda D}(\mathfrak{g}) \subset \mathbb{D}_{2}$.

16.8. Una consegnenza della definizione di germe analitico e del teorema 52 è il seguente teorema di decomposizione.

Teorema 53 (cfr. [16], p. 266).

La varietà analitica $A$ sia definita nell'intorno $U\left(P_{0}\right)$ di $P_{0}$. Esiste allora un intorno $V\left(P_{0}\right) \subset U\left(P_{0}\right)$ e un numero finito dì varietà analitiche $A_{1}, A_{2}, \ldots$ $A_{r}$ in $V\left(P_{0}\right)$, irriducibili in $P_{0}$, tali che

$$
A \cap V\left(P_{0}\right)=A_{1} \cup A_{2} \cup \ldots \cup A_{r} .
$$

Inoltre non accade mai, in alcun intorno $W\left(P_{0}\right)$ di $P_{0}$, che una delle varietò analitiche $A_{i} \cap W\left(P_{0}\right)$ sia contenuta nell'intersezione di $W\left(P_{0}\right)$ con l'unione delle altre $A_{j}(j \neq i)$.

Se nell'intorno $V^{\prime}\left(P_{0}\right)$ è data un'altra decomposizione in varietà analitiche irriducibile $A_{1}^{\prime}, A_{2}^{\prime}, \ldots, A_{s}$, si ha anzitutto $r=s$; inoltre esiste un. intorno $W\left(P_{0}\right)$ di $P_{0}$, tale che per un'opportuna scelta degli indici si abbia:

$$
A_{i}{ }^{\prime} \cap W\left(P_{0}\right)=A_{i} \cap W\left(P_{0}\right) \quad(i=1,2, \ldots, r) .
$$


Lo spezzamento di una varietà analitica $A$, cosi com' ̀̀ dato dai teoremi 52 e 53, si chiama decomposizione locale di $A$ nel punto $P_{0}$. Gli ideali primi $\mathfrak{g}_{i}(i=1,2, \ldots, r)$ sono detti ideali primi della decomposizione locale, e i germi primi $\Omega\left(\mathfrak{G}_{i}\right)$ si dicono germi primi della decomposizione locale in $P_{0}$.

COMPLEMENTI AI TEOREMI 52 E 53.

La varietà analitica $A$ sia definita dalle equazioni $f_{i}=0(i=1,2, \ldots, s)$; sia a l'ideale $\left(f_{1}, f_{2}, \ldots, f_{s}\right)$ nell'anello di serie di potenze $\mathbb{P}_{n}\left(P_{0}\right)$ e $\mathbb{D D}=\mathbb{M}(\mathfrak{a})$ il germe analitico d'insiemi di a. Ciascuna varietà analitica irriducibile $A_{i}$ della decomposizione locale di $A$ è rappresentante esattamente di un germe primo della decomposizione locale di $\mathrm{MS}$ in $F_{0}$. Inversamente ad ogni ideale primo della decomposizione locale di a in $P_{\mathrm{c}}$ corrisponde esattamente un germe primo della decomposizione locale di ID in $P_{0}$, e quest'ultimo ammette uno ed un solo rappresentante tra le varietà $A_{i}$ della decomposizione locale di $A$ in $P_{0}$.

16.9. Tra le importanti conseguenze dei teoremi di decomposizione notiamo in particolare il teorema degli zeri di HrLBERr.

Teorema 54 (Teorema degli zeri di Hilbert).

La varietà analitica $A$ sia definita in un intorno del punto $P_{0}$ dalle equazioni

$$
f_{j}=0 \quad\left(j=1,2, \ldots, s ; f_{j} \in \mathbf{D}_{n}\left(P_{0}\right)\right) ;
$$

sia $f$ una serie di potenze dell'anello $\mathbb{D}_{. .}\left(P_{0}\right)$, la quale svanisca su $A$ (cfr. 16.4). Allora esiste un'opportuna potenza $f^{\sigma}$ di $f, \sigma \geqq 1$, la quale si può porre nella forma

$$
f^{\circ}=a_{1} f_{1}+a_{2} f_{2}+\ldots+a_{s} f_{s},
$$

nella quale le $a_{i}$ sono elementi di $\mathbb{P}_{n}\left(P_{0}\right)$.

Poichè $f$ svanisce su $A$, essa è nulla anche sulle varietà analitiche irriducibili $A_{j}(j=1,2, \ldots, r)$ dello spezzamento locale di $A$ in $P_{0}$, e quindi anche sui relativi germi primi. Se poniamo $\mathfrak{g}_{j}=\mathfrak{i}\left(\Omega_{j}\right)$, ne segue che $f$ appartiene a $\mathfrak{g}_{j}(j=1,2, \ldots, r) ; f$ svanisce quindi, sulle varietà algebroidi $M\left(\mathfrak{g}_{j}\right)$ e, per la $16(2)$, sulla varietà algebroide $M(\mathfrak{a})$ dell'ideale $\mathfrak{a}=\left(f_{1}, f_{2}, \ldots, f_{\mathfrak{s}}\right)$. Ora l'affermazione segue dal teorema 32.

\section{§ 17. - Varietà analitiche monogene.}

17.1. Mediante la rappresentazione di un ideale primo per mezzo della funzione norma, troveremo un criterio per la scelta di un rappresentante di un ideale primo, il quale possegga proprietà particolarmente evidenti. La costruzione in parola si basa sulla seguente proprieta dei polinomi privilegiati. 
Sia $p(w \mid z)$ un polinomio dell' anello $\mathbb{D}_{k}[w]$, privilegiato sopra $\mathbb{D}_{k} z ;$ i coefficienti di $p$ siano convergenti nella regione cilindrica $\boldsymbol{3}(\rho)_{n}$. Per ogni $\varepsilon>0$ esistono allora numeri $\delta_{i}>0, i=1,2, \ldots, k$, con $\delta_{i}<p_{i}$, tale ohe per ogni punto $z^{0}$ di $\mathfrak{3}^{(\mathcal{C})}$, le radici del polinomio $p\left(w \mid z^{0}\right)$ giacciano tutte nel cerohio $|w|<\varepsilon$.

L'affermazione segue dal fatto che $p(w \mid z)$ diviene una potenza di $w$ quando vi si sostituisea $z_{1}=0, z_{2}=0, \ldots, z_{k}=0$, e dal teorema della continuità delle radici di un polinomio come funzione dei suoi coefficienti.

17.2. Sia $\mathfrak{g}$ un ideale primo nell'anello di serie di potenze $\mathbb{P}_{n}=\mathbb{P}_{n}(0)$; dopo la $\mathbb{P}$-trasformazione di coordinate:

$$
(y)_{n}=L(x)_{n},
$$

l'ideale $\mathfrak{g}$ possegga una base polinomiale $\bar{p}_{1}, \bar{p}_{2}, \ldots, \bar{p}_{r}$ nell'anello $\overline{\mathbb{D}}_{k, n-k}$ (cfr. Teorema 24). Sia $\overline{\mathfrak{g}}=\mathfrak{g} \cap \overline{\mathbb{P}}_{k, n-k}$ e $\Phi(\overline{\mathfrak{g}})$ sia la funzione norma di $\overline{\mathfrak{g}}$.

Nello spazio complesso di dimensione $k$ si determini la regione cilindrica

$$
\left|y_{i}\right| \leq \tau \quad(i=1,2, \ldots, k)
$$

in mode tale che in essa convergano tutti $i$ coefficienti dei polinomi $\bar{p}_{i}$ $(i=1,2, \ldots, v)$, insieme con la funzione norma. Se poi $\sigma \dot{e}$ un arbitrario numero tale che $0<\sigma<\tau$, si puó associare ad esso un numero $\rho=\rho(\sigma)$, con $0<p<\sigma$, in modo che per un arbitrario punto $(y)_{k}$ della regione cilindrica $3 k(\rho)$ :

$$
\left|y_{i}\right|<p
$$$$
(i=1,2, \ldots, k)
$$

tatti i sistemi di soluzioni delle equazioni :

$$
\bar{p}_{i}\left(y_{k+1}, y_{k+2}, \ldots, y_{n} ;(y)_{k}\right)=0 \quad(i=1,2, \ldots, r)
$$

soddisfacciano alle. disuguaglianze:

$$
\left|y_{k+j}\right|<\sigma \quad(j=1,2, \ldots, n-k) .
$$

Questa scelta di $\rho$ e possibile in base a 17.1., perchè tra $i$ polinomi $\bar{p}_{i}$ si trova un polinomio di $y_{k+j}(j=1,2, \ldots, n-k)$, che è privilegiato sopra $p_{k+j-1}$ (cfr. Teorema 24). Mediante le 17(2), 17(4) si determina cosi una regione cilindrica $3_{\sigma}$; si ponga $U_{\sigma}=L^{-1}\left(3_{\sigma}\right)$. Le $17(1), 17(3)$ definiscono in $U_{\sigma}$ una varietà analitioa $A(\mathfrak{g})$, che chiamiamo varietà analitica monogena del$l^{\prime}$ ideale primo $\mathfrak{g}$. Nelle considerazioni seguenti, per i punti di $A(\mathfrak{g})$ nseremo 
per lo più le coordinate $\left\{y_{1}, y_{2}, \ldots, y_{n}\right\}$. Paò essere inoltre utile la seguente definizione: se $P\left\{y_{1}, y_{2}, \ldots, y_{n}\right\}$ eे un punto di $A(\mathfrak{g})$, il punto dello spazio complesso di dimensione $k$, avente coordinate $\left\{y_{1}, y_{2}, \ldots, y_{k}\right\}$ si dirà proiezione di $P$.

Poichè $\sigma$ può essere scelto arbitrariamente piccolo, esistono varietà analitiche monogene di $\mathfrak{g}$ piccole quanto si vuole, cioè che giacciono in un intorno dell' origine arbitrariamente prefissato.

17.3. Per le varietà analitiche monogene si possono calcolare mediante la funzione norma le decomposizioni locali (cfr. Teorema 39).

Teorema 55.

Sia $\mathfrak{g}$ un ideale primo nell' anello delle serie convergenti di potenze $\mathbb{P}_{n}$, il quale possegga una base polinomiale nell'anello $\overline{\mathbb{D}}_{k, n-k}$ (cfr. Teorema 24). Sia $\mathfrak{g}=\mathfrak{g} \cap \overline{\mathbb{P}}_{k, n-k}$ e $\Phi(\overrightarrow{\mathfrak{g}})=\Phi\left(z, u_{k+1}, u_{k_{+2}}, \ldots, u_{n} ;(y)_{k}\right)$ sia la funzione norma di $\mathfrak{g}$. Sia $A(\mathfrak{g})$ una varietà analitica monogena di $\mathfrak{g}$ (cfr, 17.2.), definita dalle equazioni 17(1), (3) e dalle disuguaglianze 17(2) e 17(4).

Sia $\left(y^{0}\right)_{k}=\left\{y_{1}^{0}, y_{2}{ }^{0}, \ldots, y_{k^{0}}\right\}$ un sistema di $k$ numeri tali che:

$$
\left|y_{i}{ }^{0}\right|<\rho \quad(i=1,2, . ., k)(\text { cfr. } 17(2)) .
$$

Se sostituiamo $\left(y^{\circ}\right)_{k}$ al posto di $(y)_{k}$ nella $\Phi(\overline{\mathfrak{g}})$, la $\Phi\left(z, u_{k+1}, u_{k+2}, \ldots, u_{n} ;\left(y^{0}\right)_{k}\right)$ si spezza in un prodotto di fattori lineari:

$$
z-u_{k+1} y_{k+1}^{(\alpha)}-u_{k+2} y_{k+2}^{(\alpha)}-\ldots-u_{n} y_{n}^{(\alpha)} \quad(\alpha=1,2, \ldots, s)
$$

nei quali le $y_{k+1}^{(x)}, y_{k+2}^{(\alpha)}, \ldots, y_{n}^{(\alpha)}$ sono indipendenti dalle $u_{k+j} . I$ punti $P_{x}=\left\{y_{1}{ }^{0}, y_{2}{ }^{0}, \ldots, y_{k}, y_{k+1}^{(\alpha)}, y_{k+2}^{(\alpha)}, \ldots, y_{n}^{(\alpha)}\right\}(\alpha=1,2, \ldots, s)$ stanno su $A(\mathfrak{g}) e$ sono $i$ soli punti di $A(\mathfrak{g})$ che ainmeltono come proiezione $\left(y^{\circ}\right)_{k}$.

Si indichi con $\mathbb{P}_{k}{ }^{\prime}$ l'anello delle serie convergenti di potenze di $y_{1}^{\prime}=y_{1}-y_{1}{ }^{0}$, $y_{2}{ }^{\prime}=y_{2}-y_{2}{ }^{0}, \ldots, y_{k}{ }^{\prime}=y_{k}-y_{k}{ }^{0}$. Mediante prolungamento analitico di $\Phi(\overline{\mathfrak{g}})$ nel punto $\left(y^{0}\right)_{k}$ si ottiene un polinomio $\Phi^{\prime}$ dell'anello $\mathbb{D}_{k}^{\prime}\left[u_{k+1}, u_{k+2}, \ldots, u_{n}, z\right]$, il quale in generale sarà riducibile. Sia

$$
\Phi^{\prime}=\prod_{k=1}^{m} \Psi_{k}
$$

la sua decomposizione in fattori irriducibili in questo anello; il fattore $\Psi_{\mu}$ $(\mu=1,2, \ldots, m) \grave{e}$ un polinomio in $z$ con coefficiente del termine di grado massimo uguale ad 1 e avente gli altri coefficienti nell' anello $\mathbb{P}_{k}{ }^{\prime}\left[u_{k+1}, u_{k+2}\right.$, $\ldots, u_{33}$.

(a) Ciascun polinomio $\Psi_{\mu}$ è la funzione norma di un ideale primo $\overline{\mathfrak{q}}_{\mu}^{\prime}$ dell' anello $\mathbb{P}_{k}{ }^{\prime}\left[y_{k+1}, y_{k+2}, \ldots, y_{n}\right]={\overline{\mathbb{\mathbb { P } ^ { \prime }}}}_{k, n-k}$. 
(b) Mediante la sostituzione

$$
y_{1}^{\prime}=0, y_{2}^{\prime}=0, \ldots, y_{k}^{\prime}=0,
$$

ciascuno dei polinomi $\Psi_{\mu}$ si riduce ad una potenza di uno solo dei fattori lineari 17(5).

Sia dunque $\Psi_{\lambda}$ uno dei fattori $\Psi_{\mu}$ che, mediante la (17.7), danno luogo ad una potenza di (17.5). Poniamo

$$
y_{k+1}^{\prime}=y_{k+1}-y_{k+1}^{(x)}, y_{k+2}^{\prime}=y_{k+2}-y_{k+2}^{(x)}, \ldots, y_{n}^{\prime}=y_{n}-y_{n}^{(x)}
$$

e indichiamo con $\mathbb{P}_{n}^{\prime}$ l'anello di serie di potenze $\mathbb{P}\left[\left[y_{1}{ }^{\prime}, y_{2}{ }^{\prime}, \ldots, y_{n}{ }^{\prime}\right]\right]$. Sia $\mathfrak{q}_{\lambda}^{\prime}$ l'ideale esteso in $\mathbb{P}_{n}{ }^{\prime}$ dell' ideale $\overline{\mathfrak{q}}_{\lambda}{ }^{\prime}$, associato a $\Psi_{\lambda}$ secondo (a); esso è un ideale primo di $\mathbb{P}_{n}{ }^{\prime}$ e vale la relazione

$$
\overline{\mathfrak{q}}_{\lambda}^{\prime}=\mathfrak{q}_{\lambda}^{\prime} \frown \overline{\mathbb{P}}_{k, n-k}^{\prime} .
$$

(c) Sia $\mathfrak{g}^{\prime}$ il prolungamento analitico dell' ideale $\mathfrak{g}$ nel punto $P_{a}$. Siano $\Psi_{\lambda}\left(\lambda=\mu_{1}, \mu_{2}, \ldots, \mu_{h}\right)$ quei fattori $d i 17(6)$ che con la sostituzione (17.7) si riducono ad unx potenzG del fattore lineare $17(5)$. Se $\grave{e} \mathfrak{q}_{2}^{\prime}$ l'ideale associato in base $a$ (b) a $\dddot{F}_{2}$, gli ideali $\mathfrak{q}_{\text {in }}^{\prime}, \mathfrak{q}_{\mu_{2}}^{\prime}, \ldots, \mathfrak{q}_{\text {sh }_{n}}^{\prime}$ sono gli ideali primi della decomponizione locale a $i \vec{q} \mathfrak{g}^{\prime}$ nel punto $P_{x}$; in altre parole: $I$ germi analitici $\boldsymbol{\Omega}\left(\mathfrak{q}_{\lambda}{ }^{\prime}\right)\left(\lambda=\mu_{1}, \mu_{2}, \ldots, \mu_{h}\right)$ sono $i$ germi primi della decomposizione locale della varietà analitica monogena $A(\mathfrak{g})$ nel punto $P_{x}$.

La base di $\mathfrak{g}$ in $\overline{\mathbb{D}}_{k, n-k}$ sia $\bar{p}_{1}, \bar{p}_{2}, \ldots, \bar{p}_{r}$ (ofr. 17.2), i prolungamenti analitici di questi polinomi nel punto $\left(y^{0}\right)_{k}$ siano $\bar{p}_{1}^{\prime}, \bar{p}_{2}^{\prime}, \ldots, \bar{p}_{r}^{\prime}$; l'ideale generato da questi ultimi in $\overline{\mathbf{p}}_{k, n-k}^{\prime}$ sia $\overline{\mathrm{g}}^{\prime}$. La dimostrazione coincide ora con quella del teorema 39 , purchè si osservi che in corrispondenza del prolungamento analitico nel punto $\left(y^{\circ}\right)_{k}$ occorre sostituire $\mathbb{D}_{k}$ con $\mathbb{D}_{k}^{\prime}, \overline{\mathbb{D}}_{k, n-k}$ con $\overline{\mathbb{P}}_{k, n-k}^{\prime}$, $\overline{\mathbf{p}}$ con $\overline{\mathfrak{g}}^{\prime}, \overline{\mathfrak{q}}_{\mu}$ con $\overline{\mathfrak{q}}_{\mu^{\prime}}^{\prime}$ e il punto 0 col punto $P_{\alpha}$.

17.4. Tra le conseguenze del Teorema 55 è notevole la seguente proprietà dimensionale di $A(\mathfrak{g})$.

Teorema 56.

L'ideale primo $\mathfrak{g}$ dell'anello $\mathbb{P}_{n}$ abbia dimensione $k$ e sia $A(\mathfrak{g})$ una varietà analitica monogena di $\mathfrak{g}$. Allora ogni ideale primo di una decomposizione locale di $A(\mathfrak{g})$ in qualsivoglia punto di $A(\mathfrak{g})$ ha dimensione $k$.

Tale risultato ha un significato topologico.

Una varietà analitica monogena $A(\mathfrak{g})$ dell'ideale primo $\mathfrak{g}$ di dimensione $k$ $\dot{\partial}$, come insieme dello spazio complesso $C_{n}$ di dimensione $n$, un insieme $2 k$-dimensionale in ogni suo punto. 
17.5. Possiamo ora dare la dimostrazione della formula 16(4), che finora abbiamo lasciato in sospeso.

Teorema 57.

Un ideale primo dell'anello di serie di potenze determina un germe primo (cfr. 16(4)).

Per il teorema 51, tale asserzione segue dal risultato:

Sia $\mathfrak{g}$ un ideale primo dell'anello di serie di potenze $\mathbb{P}_{n}$. Se la serie $f$ di $\mathbb{P}_{n}$ non appartiene a $\mathfrak{g}$, allora $f$ non svanisce sul germe analitico $\mathbb{M}(\mathfrak{g})$ (ofr. 16.4).

Per dare una dimostrazione indiretta, ammettiamo che $f$ non sia un elemento di $\mathfrak{g}$ e tuttavia svanisea su $\mathbb{M}(\mathfrak{g})$. Ciò significa, secondo 16.4 , che esiste un rappresentante $A$ di $\mathbb{M}(\mathfrak{g})$, sul quale si ha $f=0$. L'ideale primo $\mathfrak{g}$ possegga una base polinomiale nell' anello $\overline{\mathbb{D}}_{k, n-k}$. Poichè $f$ non appartiene a $\mathfrak{g}$, esiste, per 9.2 , un polinomio

$$
g=f^{m}+c_{1}(y)_{k} f^{m-1}+\ldots+c_{n-1}(y)_{k} f+c_{m}(y)_{k}
$$

con coefficienti in $\mathbb{P}_{k}$ e $c_{\boldsymbol{m}} \neq 0$, il quale appartiene a $\mathfrak{g}$. Scegliamo una varietà analitica monogena $A(\mathfrak{g})$ e assumiamo cosi piccolo il $\sigma$ che comparisce nella definizione di $A(\mathfrak{g})$, in modo che siano soddisfatte le seguenti condizioni :

a) Le serie $c_{y}(y)_{k}(\nu=1,2, \ldots, m)$ siano convergenti nel dominio cilin. drico $3 k(\rho)$ (efr, 17(2)).

b) $A(\mathfrak{g})$ giaccia in $A$.

c) La serie $g \in \mathfrak{g}$ svanisca su $A(\mathfrak{g})$.

Da tali condizioni e dalla formula (1) segue allora che $c_{m}(y)_{k}=0$ su $\boldsymbol{A}(\mathfrak{g})$. Possiamo tuttavia trovare un punto $\left(y^{0}\right)_{k}=\left\{y_{1}{ }^{0}, y_{2}{ }^{0}, \ldots, y_{k}{ }^{0}\right\}$ in $3_{k}(\rho)$, nel quale si abbia $c_{m}\left(y_{0}\right) \neq 0$, Per il teorema 55 esistono sopra $A(\mathfrak{g})$ certi punti $P_{\alpha}(\alpha=1,2, \ldots, s)$, che hanno $\left(y^{0}\right)_{k}$ come proiezione. In tali punti si ha $c_{m} \neq 0$, in contradizione con quanto visto precedentemente.

17.6. Il nostro prossimo compito è uno studio più approfondito delle decomposizioni locali ; introduciamo a questo scopo un nuovo concetto.

Sia $A$ una varietà analitica. Il punto $P_{0}=\left(x^{0}\right)_{n}$ di $A$ si dice punto semplice di $A$, se $A$ determina in $P_{0}$ un germe analitico $\Omega_{P_{0}}$ del seguente tipo: Mediante una trasformazione pseudoconforme:

$$
z_{v}=g_{v}\left(x_{1}, x_{2}, \ldots, x_{n}\right) \quad(v=1,2, \ldots, n)
$$

di un intorno di $P_{0}$ si possa fare in modo che $\Omega_{P_{0}}$ sia rappresentato da equazioni lineari

$$
z_{k+1}=0, z_{k+2}=0, \ldots, z_{n}=0
$$


A tale definizione è equivalente la seguente condizione: Mediante una $\mathbb{P}$-trasformazione di coordinate

$$
(y)_{n}=L\left(x-x^{0}\right)_{n}
$$

si può far si che $\Omega_{P_{0}}$ sia rappresentato dalle equazioni

$$
y_{k+j}=\varphi_{k+j}(y)_{k}
$$$$
(j=1,2, \ldots, n-k)
$$

nelle quali le $千_{k+j}$ sono serie dell' anello $\mathbb{D}_{k}$.

Le equazioni $17(10)$ implicano infatti

$$
g_{k+i}\left(x_{1}, x_{2}, \ldots, x_{n}\right)=0 \quad(j=1,2, \ldots, n-k) .
$$

Poichè la $17(9)$ è pseudo conforme, esiste un sistema di variabili $x_{i_{1}}, x_{i_{2}}, \ldots$, $x_{i_{n-k}}$ tale che il determinante funzionale

$$
\frac{\partial\left(g_{k+1}, g_{k+2}, \ldots, g_{n}\right)}{\partial\left(x_{i_{1}}, x_{i_{2}}, \ldots, x_{i_{n-k}}\right)}
$$

non si annalli nel punto $\left(x^{0}\right)_{n}$. Per il teorema 46 le equazioni $17(13)$ possono allora risolversi, in un intorno di $P_{0}$, rispetto alle variabili $x_{i,}$, cioè queste variabili sono serie convergenti di potenze delle rimanenti; si ottiene cosi per $\mathbf{S D}_{P_{0}}$ una rappresentazione $17(11)$, (12).

Se viceversa è data una rappresentazione $17(11)$, (12) di $\mathbb{M}_{P_{0}}$, facciamo seguire alla $17(11)$ la seguente trasformazione:

$$
\begin{array}{cr}
z_{i}=y_{i} & (i=1,2, \ldots, k) \\
z_{k+j}=y_{k+j}-\varphi_{k+j}(y)_{k} & (j=1,2, \ldots, n-k) .
\end{array}
$$

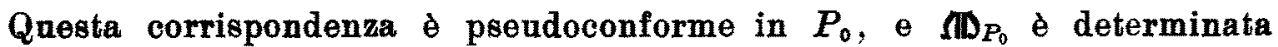
dalle $17(10)$.

17.7. Lo studio dei punti semplici delle varieta analitiche monogene si pao effettuare con profitto mediante la funzione norma. Usiamo le notazioni di 17(2). Sia $g$ un ideale primo dell' anello $D_{n}$ e $A(\mathfrak{g})$ una varietà analitica monogena di $\mathfrak{g}$. Poichè la funzione norma $\Phi(\overline{\mathfrak{g}})$ eे irriducibile, il suo discriminante $D$ rispetto a $z$ non svanisce identicamente; esso $\dot{\theta}$ un polinomio nelle $u_{k+1}, u_{k+2}, \ldots, u_{n}$ con coefficienti in $D_{k}$. Sviluppiamo $D$ secondo $i$ prodotti $U_{i}$ di potenze delle $u_{k+j}$ :

$$
D=\sum_{i=1}^{m} d_{i}(y)_{k} U_{i} .
$$


Come funzioni razionali intere dei coefficienti di $\Phi$, le serie $d_{i}$ convergono nel dominio cilindrico $3 k(\rho)$ (cfr. 17(2)); quindi mediante le equazioni

$$
d_{i}(y)_{k}=0
$$$$
(i=1,2, \ldots, m)
$$

risulta definita in $3 k(\rho)$ una varietà analitica $\Delta$, che chiamiamo "varietà di diramazione » di $A(\mathfrak{g})$, nella proiezione considerata.

\subsection{TeOREMa 58.}

Sia $A(\mathfrak{g})$ una varietà analitica monogena dell'ideale primo $\mathfrak{g}$, il quale goda delle proprietà dette in I7.2. Sia $\Delta$ la varietà di diramazione di $A(\mathfrak{g}) ;$ sia $(\bar{y})_{k}$ un punto di $\tilde{3}_{k}(\rho)-\Delta$. Allora $i$ punti di $A(\mathfrak{g})$ che hanno $(\bar{y})_{k}$ come proie. zione sono tutti distinti e sono semplici per $A(\mathfrak{g})$.

a) Per il teorema 55 il polinomio $\Phi\left(z, u_{k+1}, \ldots, u_{n} ;(\bar{y})_{k}\right)$ si spezza in un prodotto di fattori lineari 17(5); nel caso attuale queste forme lineari sono tutte diverse; perchè altrimenti il discriminante $D\left(u_{k+1}, \ldots, u_{n} ;(\tilde{y})_{k}\right)$ sarebbe nullo e quindi $(\bar{y})_{k}$ renderebbe soddisfatte le $17(15)$ ed apparterrebbe a $\Delta$.

b) A partire da $(\bar{y})_{k}$ determiniamo un sistema di valori $\left\{\bar{u}_{k_{+1}}, \bar{u}_{k_{+2}}, \ldots, \bar{u}_{n}\right\}$ tale che $\bar{D}=D\left(\bar{y}_{1}, \bar{y}_{2}, \ldots, \bar{y}_{k}, \bar{u}_{k+1}, \bar{u}_{k+2}, \ldots, \bar{u}_{n}\right) \neq 0$. Sia ora $\bar{P}=(\bar{y})_{n}$ un punto di $A(\mathfrak{g})$ che abbia per proiezione $(\bar{y})_{k}$. Si ha che

$$
\bar{z}=\bar{u}_{k+1} \bar{y}_{k+1}+\bar{u}_{k+2} \bar{y}_{k+2}+\ldots+\bar{u}_{n} \bar{y}_{n}
$$

è uno zero del polinomio $\Phi\left(z, \bar{u}_{k+1}, \bar{u}_{k+2}, \ldots, \tilde{u}_{n},(\bar{y})_{k}\right)$. Inoltre, essendo $\bar{D} \neq 0$, la derivata $\partial \Phi / \partial z$ è diversa da zero nel punto $\left\{\bar{z}, \bar{u}_{k+1}, \bar{u}_{k+2}, \ldots, \bar{u}_{n},(\bar{y})_{k}\right\}$. Per il teorema 46 esiste allora una serie di potenze $\Psi$ delle $u_{k_{+j}}^{\prime}=u_{k_{+j}}-\bar{u}_{k_{+j}}$ $(j=1,2, \ldots, n-k)$ e delle $y_{i}^{\prime}=y_{i}-\bar{y}_{i}(i=1,2, \ldots, k)$, la quale è convergente ed è tale che $\bar{z}=\Psi(0,0, \ldots, 0)$ e che $\Phi\left(\Psi, u_{k+1}, u_{k+2}, \ldots, u_{n},(y)_{k}\right) \equiv 0$. Dal teorema 55 segue che per particolari sistemi di valori $\left(y^{\prime}\right)_{k}$ del campo di convergenza la diviene una forma lineare nelle $u_{k_{+1}}, u_{k+2}, \ldots, u_{n}$. Ne segue che anche per valori $\left(y^{\prime}\right)_{k}$ generici la $\Psi$ ammette una rappresentazione

$$
\Psi=u_{k+1} \varphi_{k+1}\left(y^{\prime}\right)_{k}+u_{k+2} \varphi_{k+2}\left(y^{\prime}\right)_{k}+\ldots+u_{n} \varphi_{n}\left(y^{\prime}\right)_{k},
$$

nella quale le $\varphi_{k+j}$ sono serie di potenze dell' anello $\mathbb{p}_{k} y^{\prime}$.

La $z-\Psi$ è la funzione norma di un ideale primo, il cui germe d'insiemi ha equazioni

$$
y_{k+j}=\varphi_{k+j}\left(y^{\prime}\right)_{k} \quad(j=1,2, \ldots, n-k) .
$$

Tale germe d'insiemi è per il teorema 55 c), un germe primo dello spezza. mento locale di $A(\mathfrak{g})$ in $\bar{P}$ e, per a), è l'unico. Quindi $\bar{P}$ ’े punto semplice di $\boldsymbol{A}(\mathfrak{g})$. 
17.9. Il teorema 58 ci dà la possibilità di trovare punti semplici di $A(\mathfrak{g}$; non è detto però che si trovino così tutti i punti semplici di $A(\mathfrak{g})$, in quanto $A(\mathfrak{g})$ può possedere anche punti semplici la proiezione dei quali appartiene a $\Delta$. Vale la proprietà :

Ogni punto non semplice di $A(\mathfrak{g})$ è punto di accumulazione di punti semplici di $A(\mathfrak{g})$.

Tale asserzione segue dal teorema della continuità delle radici di un polinomio come funzione dei suoi coefficienti, applicato alla funzione norma $\Phi$, e dal fatto ohe in ogni intorno di un punto di $\Delta$ giacciono punti di $3 k(\rho)$ i quali non appartengono a $\Delta$. Tale fatto segue dal cosi detto teorema di identita per le serie di potenze:

Una serie di potenze, la quale è identicamente nulla in un intorno di un punto del suo campo di convergenza è identicamente nulla.

17.10. Vogliamo ora dare il procedimento del prolungamento analitico per i germi di insiemi delle varietà analitiche monogene $A(\mathfrak{g})$. Per quanto riguarda le notazioni si confrontino 17.2. e 17.7.

Sia $\left(y^{(1)}\right)_{k}$ un punto di $3_{k}(\rho)-\Delta$. I punti di $A(\mathfrak{g})$ che hanno $\left(y^{(1)}\right)_{k}$ come proiezione siano $P_{\lambda}(\lambda=1,2, \ldots, s)$; per il teorema 58 essi sono semplici, $\theta$ le decomposizioni locali di $A(\mathfrak{g})$ constano ciascuna di un germe primo $\Omega_{P_{\gamma}}$, che ammette una rappresentazione:

$$
y_{k+j}=\varphi_{k+j}^{(\lambda)}\left(y^{\prime}\right)_{k} \quad(j=1,2, \ldots, n-k),
$$

con $y_{i}^{\prime}=y_{i}-y_{i}^{(1)}(i=1,2, \ldots, k) ; \varphi_{k+j}^{(2)}(j=1,2, \ldots, n-k)$ è una serie di potenze dell'anello $\mathbb{D}_{k} y^{\prime}$. Sia $\hat{j}_{k}{ }^{\prime}$ un dominio cilindrico dello spazio delle $\left(y^{\prime}\right)_{k}$, nel quale convergono tutte le serie $\varphi_{k+j}^{(\lambda)}(j=1,2, \ldots, n-k ; \lambda=1,2, \ldots, s)$. La trasformazione

$$
y_{i}=y_{i}^{(1)}+y_{i}^{\prime} \quad(i=1,2, \ldots, k)
$$

muta $3 k^{\prime}$ nell' intorno $\mathfrak{U}^{(1)}$ del punto $\left(y^{(1)}\right)_{k}$ dello spazio delle $(y)_{k}$. Scegliendo $3^{\prime} k^{\prime}$ abbastanza piccolo, si può ottenere che $\mathfrak{L}^{(1)}$ giaccia in $\mathfrak{3}_{k}(\rho)-\Delta$. Le equazioni (1), (2) e la proiezione $\mathfrak{X}^{(1)}$ determinano un rappresentante $A_{P}$, del germe primo $\mathfrak{M}_{P_{\lambda}}$. Per il seguito ei sarà utile chiamare il campo $\mathfrak{U}^{(1)}$ « campo associatos al punto $\left(y^{(1)}\right)_{k}$.

Sia $\left(y^{(2)}\right)_{k}$ un altro punto di $\xi_{k}(\rho)-\Delta$. I punti di $A(\mathfrak{g})$ che hanno $\left(y^{(2)}\right)_{k}$ come proiezione siano $Q_{\mu}(\mu=1,2, \ldots, s)$. Il germe analitico di $A(\mathfrak{g})$ in $Q_{\mu}$ sia $\mathbb{M}_{Q_{\mu}}$ ed abbia la rappresentazione

$$
y_{k+j}=\Psi_{k+j}^{(\mu)}\left(y^{\prime \prime}\right)_{k} \quad(j=1,2, \ldots, n-k),
$$

con

$$
y_{i}=y_{i}^{(2)}+y_{i}^{\prime \prime} \quad(i=1,2, \ldots, k)
$$


Sia $\mathfrak{U}^{(2)}$ un campo associato a $\left(y^{(2)}\right)_{k} \in A_{Q_{k}}$ la varietà analitica definita dalle equazioni (3) e (4) e dalla proiezione $\mathfrak{U}^{(2)}$.

Supponiamo ora che $\mathfrak{L}^{(1)}$ e $\mathfrak{L}^{(2)}$ abbiano il punto (interno) $\left(y^{(3)}\right)_{k}$ in comune. Anch' esso è proiezione di punti (effettivamente) semplici $R_{\nu}(\nu=1,2, \ldots, s)$ di $A(\mathfrak{g})$. Sia $\mathbb{M}_{R_{\nu}}$ il germe analitico di $A(\mathfrak{g})$ in $R_{\nu}$, e la sua rappresentazione sia :

$$
y_{k+j}=\chi_{k+j}^{(v)}\left(y^{\prime \prime \prime}\right)_{k} \quad(j=1,2, \ldots, n-k)
$$

con

$$
y_{i}=y_{i}^{(3)}+y_{i}^{\prime \prime \prime} \quad(i=1,2, \ldots, k) .
$$

Il punto $R_{y}$ giace su una ed una sola delle $A_{P_{\lambda}}$ e su una ed una solo delle $A_{Q_{\mu}}$. A meno della numerazione dei punti $P_{\lambda}, Q_{\mu}, R_{\nu}$, possiamo sempre ottenere che per ogni $\lambda=1,2, \ldots, s$ il punto $R_{\lambda}$ giaccia su $A_{P_{\lambda}}$ e su $A_{Q_{\lambda}}$. Ora accade che $A_{P_{2}}$ determina in $R_{i}$, un germe analitico che coincide con $\mathbb{M}_{R_{\gamma}}$ e la cui rappresentazione si può ottenere prolungando analiticamente le serie $\varphi_{k+j}^{(\lambda)}$ nel punto $\left\{y_{1}^{(3)}-y_{1}^{(1)}, y_{2}^{(3)}-y_{2}^{(1)}, \ldots, y_{k}{ }^{(3)}-y_{k}{ }^{(1)}\right\}$; denotiamo tale prolungamento analitico con $\varphi_{k+j}^{(\lambda)}\left\{R_{\lambda}\right\}$; esso è una serie di potenze nelle variabili $\left\{y_{1}^{\prime \prime \prime}, y_{2}^{\prime \prime \prime}, \ldots, y_{k}^{\prime \prime \prime}\right\}$. Dal teorema di identità per le serie di potenze segue, per ogni $\lambda=1,2, \ldots, s$ :

$$
\varphi_{k \rightarrow j}^{(\lambda)}\left\{R_{,}\right\}=\chi_{k+j}^{(\lambda)} \quad(j=1,2, \ldots, n-k) .
$$

Analogamente $A_{Q}$, determina in $R_{2}$ un germe analitico che coincide con $\boldsymbol{M S}_{R_{\lambda}}$ e che si ottiene da (3). mediante prolungamento analitico delle serie $\Psi_{k+j}^{(\lambda)}$ nel punto $\left\{y_{1}^{(3)}-y_{1}{ }^{(2)}, y_{2}{ }^{(3)}-y_{2}{ }^{(2)}, \ldots, y_{k}{ }^{(3)}-y_{k}{ }^{(2)}\right\}$. Sorgono così le $\Psi_{k+j}^{(\lambda)}\left\{R_{\lambda}\right\}$ e si ha, per ogni $\left.\lambda=1,2, \ldots, s\right)$ :

$$
\Psi_{k+j}^{(\lambda)}\left\{R_{\lambda}\right\}=\chi_{k+j}^{(\lambda)} \quad(j=1,2, \ldots, n-k) .
$$

Mediante le relazioni (7) e (8) le varietà analitiche $A_{P_{\lambda}}$ e $A_{Q_{\gamma}}$, e quindi anche i germi primi $\mathbb{M}_{P_{\gamma}}$ e $\mathbb{M}_{Q_{\lambda}}$, vengono saldate tra loro; come nella teoria delle funzioni, esprimeremo tale fatto dicendo che $\mathbb{M}_{P_{\gamma}}$ e $\mathbb{M}_{Q_{\lambda}}$ si ottengono l'uno dall'altro mediante prolungamento analitico. Tale relazione si può gene. ralizzare alla maniera seguente, che è valida del resto anche nella teoria delle funzioni :

Siano $P$ e $Q$ punti semplici di $A(\mathfrak{g})$, coi germi primi $\Omega_{P}, \mathbb{D}_{Q}$ rispettivamente. Esista una successione finita di punti semplici $P_{1}, P_{2}, \ldots, P_{h}$ di $A(\mathfrak{g})$, tale che $P_{1}=P, P_{h}=Q$ e che il germe primo $\int \mathbb{D}_{P_{i}}$ di $A(\mathfrak{g})$ in $P_{i}$ sia prolungamento analitico del germe primo $\mathbb{M}_{P_{i-1}}$ di $A(\mathfrak{g})$ in $P_{i-1}$.

Per distinguere quest' ultimo tipo di prolungamento analitico da quello più sopra introdotto (15.6), si chiama quello «prolungamento analitico diretto». 
17.11. La potenza del procedimento del prolungamento analitico si osserva in particolare modo quando si prendano le mosse dalle curve tracciate in $3_{k}(\rho)-\Delta$. Sia $\Gamma$ una di esse e siano $\alpha$ e $\beta$ i suoi estremi. Sia $P$ un punto di $A(\mathfrak{g})$ che abbia per proiezione $\alpha$ e $\mathbb{M}_{P}$ il germe primo di $A(\mathfrak{g})$ in $P$. Definiamo il prolungamento analitico di $\mathbb{M D}_{P}$ lungo la curva $\Gamma$. Ad ogni punto di $\Gamma$ associamo un campo secondo il n. 17.10.; per il teorema di ricoprimento di HeInE-Borel esiste un numero finito di punti $\left(y^{(i)}\right)_{k}(i=1,2, \ldots, h)$ tali che $i$ campi ad essi associati ricoprano interamente $\Gamma$. Possiamo supporre anche che sia $\left(y^{(1)}\right)_{k}=\alpha$ e $\left(y^{(h)}\right)_{k}=\beta$. Come in 17.10. ci si persuade facilmente che per ogni $i$ esiste in $A(\mathfrak{g})$ un punto $P_{i}$ che ha per proiezione $\left(y^{(t)}\right)_{k}$ in modo tale che $P_{1}=P$ e che il germe primo $\Omega_{P_{i}}$ sia prolungamento analitico del germe primo $\mathfrak{M}_{P_{i-1}}(i=2, \ldots, h)$. Si dice allora che $\mathbb{M}_{P_{h}}$ è prolungamento analitico di $\Omega D_{P}$ lungo la curva $\Gamma$.

Occorre dimostrare che tale procedimento è indipendente dalla sceltá dei punti intermedi su $\Gamma$. Come nell'usuale teoria delle funzioni, la dimostrazione segue facilmente dall' univocità del prolungamento analitico diretto.

17.12. Ci poniamo ora il problema di determinare quali sono $i$ germi analitici di $A(\mathfrak{g})$ che si possono conseguire come prolungamenti analitici a partire da un assegnato germe primo di $A(\mathfrak{g})$. All' uopo è importante sapere che $3 k(\rho)-\Delta$ risulta connesso per mezzo di curve, come risulta dal seguente teorema :

TEOREMA 59.

La serie di potenze $f(x)_{n}$ sia convergente nel campo cilindrico 3. Se si denota con $A$ la varietà analitica $f=0$ in 3 , allora $3-A$ risulta connesso mediante curve.

Il campo cilindrico 3 è convesso, vale a dire che se due punti appartengono ad esso, vi appartiene anche il segmento che li congiunge. Siano ora $P_{1}=\left(x^{(1)}\right)_{n}$ e $P_{2}=\left(x^{(2)}\right)_{n}$ due punti di $3-A$. Sostituendo in $f$ le espressioni

$$
x_{i}=x_{i}^{(1)}+t\left(x_{i}^{(2)}-x_{i}^{(1)}\right) \quad(i=1,2, \ldots, n) .
$$

si ottiene una funzione $f^{*}(t)$ con $f^{*}(0) \neq 0$ e $f^{*}(1) \neq 0$. La funzione $f^{*}(t)$, in conseguenza del Teorema 45 , è in ogni caso olomorfa in tutti quei punti del piano della $t$, che mediante la formula (1) determinano punti di 3 , in particolare quindi anche nei punti del segmento congiungente i punti $t=0 \mathrm{e}$ $t=1$. Per $i$ teoremi della teoria delle funzioni tale segmento possiede percio nel piano della $t$ un intorno $V$ p. es. persino un rettangolo, nell' involucro chiuso $\bar{V}$ del quale la $f^{*}(t)$ è ancora olomorfa. Essa vi può avere perciò solo nn numero finito di zeri, e si può trovare facilmente in $\bar{V}$ una curva congiungente i punti $t=0$ e $t=1$, la quale non incontra alcuno degli zeri di 
$f^{*} t$ ) e che viene mutata dalle (1) in una curva $\Gamma$ di $3 ; \Gamma$ giace allora in 3-A e congiunge $P_{1}$ e $P_{2}$.

17.13. Scegliamo il punto $\alpha$ in $3 k(\rho)-\Delta$. I punti di $A(\mathfrak{g})$ che hanno $\alpha$ come proiezione siano $P_{\lambda}(\lambda=1,2, \ldots, s)$. Sia $\Omega_{P_{\lambda}}$ il germe primo di $A(\mathfrak{g})$ in $P_{\lambda}$.

Sia $\beta$ un altro punto di $3 k(\rho)-\Delta$, siano $Q_{\mu}(\mu=1,2, \ldots, s)$ i punti di $A(\mathfrak{g})$ che hanno $\beta$ come proiezione e $\mathbb{M}_{Q_{1,}}$ i germi primi in essi definiti da $A(\mathfrak{g})$. Lungo una curva $\Gamma$ tracciata in $3 k(\rho)-\Delta$, che congiunge $\alpha$ e $\beta$, ogni germe primo $\mathbb{M}_{P_{\lambda}}$ dà, prolungato analiticamente, uno dei germi primi $\mathbb{D}_{Q_{\mu}}$, e vice. versa; la curva $\Gamma$ stabilisce quindi una corrispondenza biunivoca tra gli $\mathbb{M}_{P_{\lambda}}$ e gli $\Omega_{Q_{\mu}}$. La più importante questione che si pone è la seguente: Si pud scegliere $\Gamma$ in modo tale che un prefissato $\Omega_{P_{2}}$ si muti in un pre. fissato $\int_{Q_{\mu}}$ ? Come segue dal teorema 59 , basta ridursi al caso in cui sia $\alpha=\beta$, nel qual caso il problema si riduce a quello di determinare se esiste, per due prefissati valori $\lambda$ e $\lambda^{\prime}$, una curva chiusa uscente da $\propto$ e tracciata in $3_{k}(\rho)-\Delta$, lungo la quale $\Omega_{P_{\lambda}}$ risulti come prolungamento analitico di $\Omega_{P_{2^{\prime}}}$.

Mediante opportuna numerazione dei punti $P_{\lambda}$ si può ottenere che i germi primi $\mathfrak{M}_{P_{1}}, \mathfrak{S}_{P_{2}}, \ldots, \mathfrak{M}_{P_{h}}$ siano analiticamente legati nel modo detto, mentre nessuno dei germi primi $\Omega_{P_{\lambda}}$ con $\lambda>h$ si può ottenere come pro. lungamento analitico dei precedenti. La rappresentazione di $\boldsymbol{\Omega}_{P_{\lambda}}$ mediante serie di potenze sia

$$
y_{k+j}=\varphi_{k+j}^{(2)} \quad(j=1,2, \ldots, n-k) .
$$

Formiamo il seguente polinomio nelle $z, u_{k_{+1}}, u_{k+2}, \ldots, u_{n}$ :

$$
\Psi=\prod_{\lambda=2}^{h}\left(z-u_{k+1} \varphi_{k+1}^{(\lambda)}-u_{k+2} \varphi_{k+2}^{(\lambda)}-\ldots-u_{n} \varphi_{n}^{(\lambda)}\right) .
$$

I coefficienti di $\Psi$ sono olomorfi nelle $\left\{y_{1}, y_{2}, \ldots, y_{k}\right\}$ in un intorno di $\alpha$; li possiamo inoltre prolungare analiticamente lungo un cammino arbitrario in $3 k(\rho)-\Delta$, perchè tale prolungamento analitico è possibile per i sistemi (1). Tale prolungamento $\dot{e}$ pure univoco in $z_{k}(\rho)-\Delta$, già indipendente dal cam. mino scelto, perchè il polinomio $\Psi$ si muta in sè in ogni prolungamento analitico effettuato lungo un cammino chiuso di $3 k^{\prime}(p)-\Delta$.

Per poter trarre la conclusione finale $h=s$ ei occorrono due teoremi delle funzioni di più variabili complesse, le dimostrazioni dei quali non riportiamo qui.

I. Teorema di RIEManN sulle singollaritì Rimovibili. - (Cfr. [15], pag. 186).

La funzione $f\left(y_{1}, y_{2}, \ldots, y_{k}\right)$ sia olomorfa nell'intorno $U$ del punto $y^{0}$ con 
eccezione eventualmente di punti giacenti sopra una varietà analitica $\delta$ di $U$. di una dimensione $<k$; inoltre il valore assoluto di $f$ sia limitato in $U-\bar{o}$. Allora si pù̀ completare la definizione di $f$ nei punti di ó in modo tale che essa risulti olomorfa in $y^{\circ} ;$ in altre parole, $i$ punti $d i \delta$ si presentano come singolaritò rimovibili.

II. Teorema delle serie di Cauchy-Taylor. - (Cfr. [15], pag. 49).

La funzione $f\left(y_{1}, y_{2}, \ldots, y_{k}\right)$ sia olomorfa nel campo cilindrico 3. Allora $f$ possiede una rappresentazione come serie di potenze dell'anello $\mathbb{P}_{k} y$, convergente in 3 .

Poichè per tutti i punti di $A(\mathfrak{g})$ valgono le disuguaglianze $17(4)$, si ha in $3_{k}(\rho)-\Delta:$

$$
\left|\varphi_{k+j}^{(\lambda)}\right| \leq \sigma \quad(j=1,2, \ldots, n-k ; \lambda=1,2, \ldots, s) .
$$

Il teorema di Rremann è applicabile ai coefficienti di $\Phi$, che sono olomorfi in $3 k(\rho)-\Delta$ e per la (2) anche limitati. Questi coefficienti sono dunque olomorfi in $3 k$ e per il teorema di CAUCHY-TAYLOR risultano allora serie di potenze dell'anello $\mathbb{P}_{k} y$, convergenti in $3_{k}(p)$. Se fosse $h<s$. il polinomio $\Psi$ sarebbe un divisore proprio della funzione norma $\Phi(\overline{\mathfrak{g}})$ nell'anello $\mathbb{D}_{k}\left[\boldsymbol{u}_{k_{+1}}\right.$, $\left.u_{k+2}, \ldots, u_{n}, z\right]$; ma un tal divisore non esiste per l'irriducibilità di $\Phi(\overline{\mathfrak{g}})$; si ha quindi $h=s$. I risultati visti si possono così riassumere:

Tutti $i$ germi primi di $A(\mathfrak{g})$ nei suoi punti semplici si possono ricondurre l'uno all'altro mediante prolungamento analitico.

$\mathrm{Ne}$ segue in particolare:

Teorema 60.

Sia $\mathfrak{g}$ un ideale primo dell'anello di serie di potenze $\mathbb{p}_{n}$ e $A(\mathfrak{g})$ una varietà analitica monogena di $\mathfrak{g}$. L' insieme dei punti semplici di $A(\mathfrak{g})$ è connesso mediante curve.

17.14. Per estendere i risultati del n. 17.13. ai germi primi nei punti non semplici, occorre un'estensione a tali germi del concetto di prolungamento analitico.

Sia $\mathbb{D}_{P}$ un germe primo del punto $P$ e $\mathbb{I D}_{Q}$ un germe primo del punto $Q$. Sia $A_{P}$ una varietà analitica monogena, rappresentante di $\mathbb{M}_{P}$, e $A_{Q}$ una varietà monogena di $\mathfrak{\Omega D}_{Q}$. Esista un punto $R$, giacente su $A_{P}$ e su $A_{Q}$, e punto semplice per entrambe. Se il germe primo di $A_{P}$ in $R$ coincide col germe primo di $A_{Q}$ in $R$, diciamo che $\mathbb{D}_{P}$ e $\mathbb{N}_{Q}$ sono prolungamento analitico l'uno dell'altro; come prolungamento analitico viene considerata anche l'operazione che si ottiene ripetendo un numero finito di volte il procedimento precedente. E facile verificare che la definizione data in 17.10. è un caso particolare della presente. 
Da tale definizione e dal risultato di 17.13. segue:

Teorema 61.

Sia $\mathfrak{g}$ un ideale primo dell'anello di serie di potenze $\mathbb{P}_{n}$ e $A(\mathfrak{g})$ una varietà analitica monogena di $\mathfrak{g}$ (cfr. 17.2). Due qualsiansi germi primi di decomposizioni locali di $A(\mathfrak{g})$ si ottengono l'uno dall'altro mediante prolungamento analitico.

Il teorema 61 chiarisce la ragione del termine «monogeno» che abbiamo riservato alle varietà del tipo di $A(\mathfrak{g})$, in quando prendendo le mosse da un qualsiasi germe primo si può generare tutta la $A(\mathfrak{g})$ mediante prolungamento analitico.

17.15. Con la monogeneità $\dot{\theta}$ collegata un'altra proprietà dei germi primi delle decomposizioni locali di $A(\mathfrak{g})$, cioè il carattere di punti «generali » che essi hanno, nel senso che segue (cfr. 14.7.):

Teorema 62.

Sia $\mathfrak{g}$ un ideale primo dell'anello di serie di potenze $\mathbb{D}_{n}$ e $A(\mathfrak{g})$ una varietì analitica monogena (cfr. 17.2). Sia $f\left(x_{1}, x_{2}, \ldots, x_{n}\right)$ una serie di $\mathbb{\mathbb { P }}_{n}$, il cui campo di convergenza contiene il campo $U_{0}$ nel quale è definita $A(\mathfrak{g})(c f i .17 .2)$; la funzione $f$ svanisca sopra un germe primo di una decomposizione locale in un punto qualsiasi di $A(\mathfrak{g})$. Allora $f$ svanisce identicamente sopra $A(\mathfrak{g})$; dal teorema 57 segue inoltre che $f$ appartiene $a \mathfrak{g}$.

Sia $\Omega_{P}$ il germe primo di $A(\mathfrak{g})$, sul quale svanisce la $f$. Esiste allora una varieta analitica monogena $A_{P}$, rappresentante di $\mathbb{M}_{P}$, sulla quale e $f=0$. Sia $\Omega \mathbb{D}_{Q}$ il germe primo della decomposizione locale di $A_{P}$ in un punto semplice $Q$ di $A_{P}$. Se scegliamo $A_{P}$ cosi piccola che si abbia $A_{P} \subset A(\mathfrak{g})$, per il teorema 55 risulta che $\mathfrak{M D}_{Q}$ è anche il germe primo di $A(\mathfrak{g})$ in $Q$; esso sia rappresentato dalle serie di potenze:

$$
y_{k+j}=\varphi_{k+j}(y)_{k} \quad(j=1,2, \ldots, n-k) .
$$

Poichè $f$ svanisce su $A_{P}$, sostituendo tali serie in $f$ si ha:

$$
f\left(y_{1}, y_{2}, \ldots, y_{k}, f_{k+1}, \varphi_{k+2}, \ldots, \varphi_{n}\right) \equiv 0 \text {. }
$$

Nel prolungamento analitico del sistema (1) in $3 k(\rho)-\Delta$ la relazione (2) rimane valida e dal teorema 61 segue la tesi.

17.16. Il teorema 62 ammette la seguente generalizzazione.

Thorema 63, (cfr. [8], § 2, IV.

Sia A una varietà analitica definita nell'intorno $U$ del punto 0 , la quale determini in 0 un germe analitico $\mathbb{M}$. 
Sia $\mathbb{Q}$ un germe primo del punto 0 e $A_{0}$ una varielà analitica monogena $d i \mathbb{Q}$, appartenente ad $U$.

Sia dato su $A$ e su $A_{0}$ un punto $P$ con la seguente proprietà: Un germe primo della decomposizione locale di $A_{0}$ in $P$ giaccia sopra un germe primo della decomposizione locale di $A$ in $P$. Allora $\mathbb{Q}$ appartiene ad $\mathbb{\Omega}$,

Le equazioni di $A$ in $U$ siano

$$
f_{i}=0, \quad f_{i} \in \mathbb{P}_{n}, \quad(i=1,2, \ldots, s) .
$$

Dal teorema 62 segue che le funzioni $f_{i}$ svanisoono su $A_{0}$ ed appartengono all'ideale primo di $\mathbb{Q}$. Ne segue la tesi.

\section{BIBLIOGRAFIA}

[1] Bochner, S. and Martin, W. T., Several complex variables, Princeton (1948).

[2] Bourbakr, N., Algèbre, IV, Paris (1850).

[3] CoHan, I. S., On the structure and ideal theory of complete local vings, "Trans. Amer. Math. Soc. ", 59 (1946).

[4] GröbNER, W., Moderne algebraische Geometrie, Wien und Innsbruck (1949).

[5] Hodge, W. V. D. und Pgdoe, D., Methods of algebraic geometry, Vol. I, Cambridge (1947).

[6] - -, Methods of algebraic, geometry, Vol. II, Cambridge (1952).

[7] - -, Methods of algebraic geometry, Vol. III, Cambridge (1954).

[8] Kraser, H., Analytische Mannigfaltigkeiten im komplexen projektiven Raum, Math. Nachr. *, $4(1050)$.

[9] Kxopp, K., Theorie und Anwendung der unendlichen Reihen, 4. Aufl., Berlin-Göttingen-Heidelberg (1947).

[10] KnuLL, W., Zur Arithmetik der Potenzreihen mit ganzzahligen Koeffizienten, *Journ. f. d. reine a. angew. Math. ', 164 (1931).

[11] - -, Potenereikenringe, Math. Zeitschr. *, 43 (1938).

[12] Lifschetz, S., Algebraic geometry, Princeton (1953).

[18] Lefschetz, S. und Whitmeread, On analytical complexes, * Transact. Amer. Math. Soc. *, 85 (1933).

[14] Northcotr, D. G.. Ideal Theory, Cambridge (1853).

[15] OsGooD, W. F., Lehrbuch der Funletionentheorie, Bd. II, 1. Teil, 2. Aufl. Leipzig u. Berlin (1929).

[16] Rmmmert R. und Stmin, K., Über aie wesentlichen Singularitäten analytischer Mengen, - Math. Ann. ", 126 (1953).

[17] Rucknrs, W., Zum Elimimationsproblem der Potenzreihenideale, * Math. Ann. ", 107 (1932).

[18] SamuzL, P. Mdthodes $d^{\prime}$ algbore abstraite en gémotrie algbbrique, Berlin-Gbitingen-Heidelberg (1955),

[19] จ. D. W ARRDEN, B. L. Über irredueible algebraische Mannigfaltigkeiten. "Math. Ann. ", 108 (1833).

[20] - -, Einführwing in dis algebraische Goometrie, Berlin (1989).

[21] WaIL, A., Froundations of algebraic geometry, New York (1946). 